\title{
Introduction to quantum noise, measurement, and amplification
}

\author{
A. A. Clerk* \\ Department of Physics, McGill University, 3600 rue University Montréal, Quebec, Canada \\ H3A 2 T8
}

\section{H. Devoret}

Department of Applied Physics, Yale University, P.O. Box 208284, New Haven, Connecticut 06520-8284, USA

\section{S. M. Girvin}

Department of Physics, Yale University, P.O. Box 208120, New Haven, Connecticut 06520-8120, USA

\section{Florian Marquardt}

Department of Physics, Center for NanoScience, and Arnold Sommerfeld Center for Theoretical Physics, Ludwig-Maximilians-Universität München, Theresienstrasse 37, D-80333 München, Germany

\section{R. J. Schoelkopf}

Department of Applied Physics, Yale University, P.O. Box 208284, New Haven, Connecticut 06520-8284, USA

(Published 15 April 2010)

\begin{abstract}
The topic of quantum noise has become extremely timely due to the rise of quantum information physics and the resulting interchange of ideas between the condensed matter and atomic, molecular, optical-quantum optics communities. This review gives a pedagogical introduction to the physics of quantum noise and its connections to quantum measurement and quantum amplification. After introducing quantum noise spectra and methods for their detection, the basics of weak continuous measurements are described. Particular attention is given to the treatment of the standard quantum limit on linear amplifiers and position detectors within a general linear-response framework. This approach is shown how it relates to the standard Haus-Caves quantum limit for a bosonic amplifier known in quantum optics and its application to the case of electrical circuits is illustrated, including mesoscopic detectors and resonant cavity detectors.
\end{abstract}

DOI: 10.1103/RevModPhys.82.1155

PACS number(s): $72.70 .+\mathrm{m}$

\section{CONTENTS}

I. Introduction

1156

II. Quantum Noise Spectra

A. Introduction to quantum noise

B. Quantum spectrum analyzers

III. Quantum Measurements

A. Weak continuous measurements

B. Measurement with a parametrically coupled resonant cavity

1. QND measurement of the state of a qubit using a resonant cavity

2. Quantum limit relation for QND qubit state detection

3. Measurement of oscillator position using a resonant cavity

1169

IV. General Linear-Response Theory

A. Quantum constraints on noise

\footnotetext{
*clerk@physics.mcgill.ca
}

1. Heuristic weak-measurement noise constraints 1173

2. Generic linear-response detector 1174

3. Quantum constraint on noise 1175

4. Evading the detector quantum noise inequality 1176

B. Quantum limit on QND detection of a qubit 1177

V. Quantum Limit on Linear Amplifiers and Position Detectors

A. Preliminaries on amplification

B. Standard Haus-Caves derivation of the quantum limit on a bosonic amplifier 1179

C. Nondegenerate parametric amplifier 1181

1. Gain and added noise 1181

2. Bandwidth-gain trade-off 1182

3. Effective temperature 1182

D. Scattering versus op-amp modes of operation 1183

E. Linear-response description of a position detector 1185

1. Detector back-action 1185

2. Total output noise 1186

3. Detector power gain 1186

4. Simplifications for a quantum-ideal detector 1188 
5. Quantum limit on added noise and noise temperature

F. Quantum limit on the noise temperature of a voltage amplifier

1. Classical description of a voltage amplifier

2. Linear-response description

3. Role of noise cross correlations

G. Near quantum-limited mesoscopic detectors

1. dc superconducting quantum interference device amplifiers

2. Quantum point contact detectors

3. Single-electron transistors and resonant-level detectors

H. Back-action evasion and noise-free amplification

1. Degenerate parametric amplifier

2. Double-sideband cavity detector

3. Stroboscopic measurements

VI. Bosonic Scattering Description of a Two-Port Amplifier

A. Scattering versus op-amp representations

1. Scattering representation

2. Op-amp representation

3. Converting between representations

B. Minimal two-port scattering amplifier

1. Scattering versus op-amp quantum limit

2. Why is the op-amp quantum limit not achieved?

VII. Reaching the Quantum Limit in Practice

A. Importance of QND measurements

B. Power matching versus noise matching

VIII. Conclusions

Acknowledgments

References

\section{INTRODUCTION}

Recently several advances have led to a renewed interest in the quantum-mechanical aspects of noise in mesoscopic electrical circuits, detectors, and amplifiers. One motivation is that such systems can operate simultaneously at high frequencies and at low temperatures, entering the regime where $\hbar \omega>k_{B} T$. As such, quantum zero-point fluctuations will play a more dominant role in determining their behavior than the more familiar thermal fluctuations. A second motivation comes from the relation between quantum noise and quantum measurement. There exists an ever-increasing number of experiments in mesoscopic electronics where one is forced to think about the quantum mechanics of the detection process, and about fundamental quantum limits which constrain the performance of the detector or amplifier used.

Given the above, we will focus in this review on discussing what is known as the "standard quantum limit" (SQL) on both displacement detection and amplification. To preclude any possible confusion, it is worthwhile to state explicitly from the start that there is no limit to how well one may resolve the position of a particle in an instantaneous measurement. Indeed, in the typical Heisenberg microscope setup, one would scatter pho- tons off an electron, thereby detecting its position to an accuracy set by the wavelength of photons used. The fact that its momentum will suffer a large uncontrolled perturbation, affecting its future motion, is of no concern here. Only as one tries to further increase the resolution will one finally encounter relativistic effects (pair production) that set a limit given by the Compton wavelength of the electron. The situation is obviously very different if one attempts to observe the whole trajectory of the particle. As this effectively amounts to measurements of both position and momentum, there has to be a trade-off between the accuracies of both, set by the Heisenberg uncertainty relation. This is enforced in practice by the uncontrolled perturbation of the momentum during one position measurement adding to the noise in later measurements, a phenomenon known as "measurement back-action."

Just such a situation is encountered in "weak measurements" (Braginsky and Khalili, 1992), where one integrates the signal over time, gradually learning more about the system being measured; this review will focus on such measurements. There are many good reasons why one may be interested in doing a weak measurement, rather than an instantaneous, strong, projective measurement. On a practical level, there may be limitations to the strength of the coupling between the system and the detector, which have to be compensated by integrating the signal over time. One may also deliberately opt not to disturb the system too strongly, e.g., to be able to apply quantum feedback techniques for state control. Moreover, as one reads out an oscillatory signal over time, one effectively filters away noise (e.g., of a technical nature) at other frequencies. Finally, consider an example like detection of the collective coordinate of motion of a micromechanical beam. Its zero-point uncertainty (ground-state position fluctuation) is typically on the order of the diameter of a proton. It is out of the question to reach this accuracy in an instantaneous measurement by scattering photons of such a small wavelength off the structure, since they would instead resolve the much larger position fluctuations of the individual atoms comprising the beam (and induce all kinds of unwanted damage), instead of reading out the center-of-mass coordinate. The same holds true for other collective degrees of freedom.

The prototypical example we discuss is that of a weak measurement detecting the motion of a harmonic oscillator (such as a mechanical beam). The measurement then actually follows the slow evolution of amplitude and phase of the oscillations (or, equivalently, the two quadrature components), and the SQL derives from the fact that these two observables do not commute. It essentially says that the measurement accuracy will be limited to resolving both quadratures down to the scale of the ground-state position fluctuations, within one mechanical damping time. Note that, in special applications, one might be interested only in one particular quadrature of motion. Then the Heisenberg uncertainty relation does not enforce any SQL and one may again 
obtain unlimited accuracy, at the expense of renouncing all knowledge of the other quadrature.

Position detection by weak measurement essentially amounts to amplification of the quantum signal up to a classically accessible level. Therefore, the theory of quantum limits on displacement detection is intimately connected to limits on how well an amplifier can work. If an amplifier does not have any preference for any particular phase of the oscillatory signal, it is called "phase preserving," which is the case relevant for amplifying and thereby detecting both quadratures equally well. ${ }^{1}$ We derive and discuss the SQL for phase-preserving linear amplifiers (Haus and Mullen, 1962; Caves, 1982). Quantum mechanics demands that such an amplifier adds noise that corresponds to half a photon added to each mode of the input signal, in the limit of high photon-number gain $G$. In contrast, for small gain, the minimum number of added noise quanta, $(1-1 / G) / 2$, can become arbitrarily small as the gain is reduced down to 1 (no amplification). One might ask, therefore, whether it should not be possible to evade the SQL by being content with small gains. The answer is no, since high gains $G \gg 1$ are needed to amplify the signal to a level where it can be read out (or further amplified) using classical devices without their noise having any further appreciable effect, converting 1 input photon into $G \gg 1$ output photons. According to Caves, it is necessary to generate an output that "we can lay our grubby, classical hands on" (Caves, 1982). It is a simple exercise to show that feeding the input of a first, potentially lowgain amplifier into a second amplifier results in an overall bound on the added noise that is just the one expected for the product of their respective gains. Therefore, as one approaches the classical level, i.e., large overall gains, the SQL always applies in its simplified form of half a photon added.

Unlike traditional discussions of the amplifier SQL, here we devote considerable attention to a general linear-response approach based on the quantum relation between susceptibilities and noise. This approach treats the amplifier or detector as a black box with an input port coupling to the signal source and an output port to access the amplified signal. It is more suited for mesoscopic systems than the quantum optics scattering-type approach, and it leads us to the quantum noise inequality: a relation between the noise added to the output and the back-action noise feeding back to the signal source. In the ideal case (what we term a "quantum-limited detector"), the product of these two contributions reaches the minimum value allowed by quantum mechanics. We show that optimizing this inequality on noise is a necessary prerequisite for having a detector achieve the quantum limit in a specific measurement task, such as linear amplification.

There are several motivations for understanding in

\footnotetext{
${ }^{1}$ In the literature this is often referred to as a "phase insensitive" amplifier. We prefer the term "phase preserving" to avoid any ambiguity.
}

principle, and realizing in practice, amplifiers whose noise reaches this minimum quantum limit. Achieving the quantum limit on continuous position detection has been one of the goals of many recent experiments on quantum electromechanical (Cleland et al., 2002; Knobel and Cleland, 2003; LaHaye et al., 2004; Naik et al., 2006; Flowers-Jacobs et al., 2007; Etaki et al., 2008; Poggio et al., 2008; Regal et al., 2008) and optomechanical systems (Arcizet et al., 2006; Gigan et al., 2006; Schliesser et al., 2008; Thompson et al., 2008; Marquardt and Girvin, 2009). As we will show, having a near-quantum-limited detector would allow one to continuously monitor the quantum zero-point fluctuations of a mechanical resonator. It is also necessary to have a quantum-limited detector is for such tasks as single-spin NMR detection $(\mathrm{Ru}-$ gar et al., 2004), as well as gravitational wave detection (Abramovici et al., 1992). The topic of quantum-limited detection is also directly relevant to recent activity exploring feedback control of quantum systems (Wiseman and Milburn, 1993, 1994; Doherty et al., 2000; Korotkov, 2001b; Ruskov and Korotkov, 2002); such schemes require need a close-to-quantum-limited detector.

This review is organized as follows. We start in Sec. II by providing a review of the basic statistical properties of quantum noise, including its detection. In Sec. III we turn to quantum measurements and give a basic introduction to weak continuous measurements. To make things concrete, we discuss heuristically measurements of both a qubit and an oscillator using a simple resonant cavity detector, giving an idea of the origin of the quantum limit in each case. Section IV is devoted to a more rigorous treatment of quantum constraints on noise arising from general quantum linear-response theory. The heart of the review is contained in Sec. V, where we give a thorough discussion of quantum limits on amplification and continuous position detection. We also discuss various methods for beating the usual quantum limits on added noise using back-action evasion techniques. We are careful to distinguish two very distinct modes of amplifier operation (the "scattering" versus "op-amp" modes); we expand on this in Sec. VI, where we discuss both modes of operation in a simple two-port bosonic amplifier. Importantly, we show that an amplifier can be quantum limited in one mode of operation, but fail to be quantum limited in the other mode of operation. Finally, in Sec. VII we highlight a number of practical considerations that one must keep in mind when trying to perform a quantum-limited measurement. Table I provides a synopsis of the main results discussed in the text as well as definitions of symbols used.

In addition to the above, we have supplemented the main text with several pedagogical appendixes that cover basic background topics. Particular attention is given to the quantum mechanics of transmission lines and driven electromagnetic cavities, topics that are especially relevant given recent experiments making use of microwave stripline resonators. These appendixes are contained in a separate online-only supplementary document (Clerk et al., 2009) (see also http://arxiv.org/ abs/0810.4729). In Table II, we list the contents of these 
TABLE I. Table of symbols and main results.

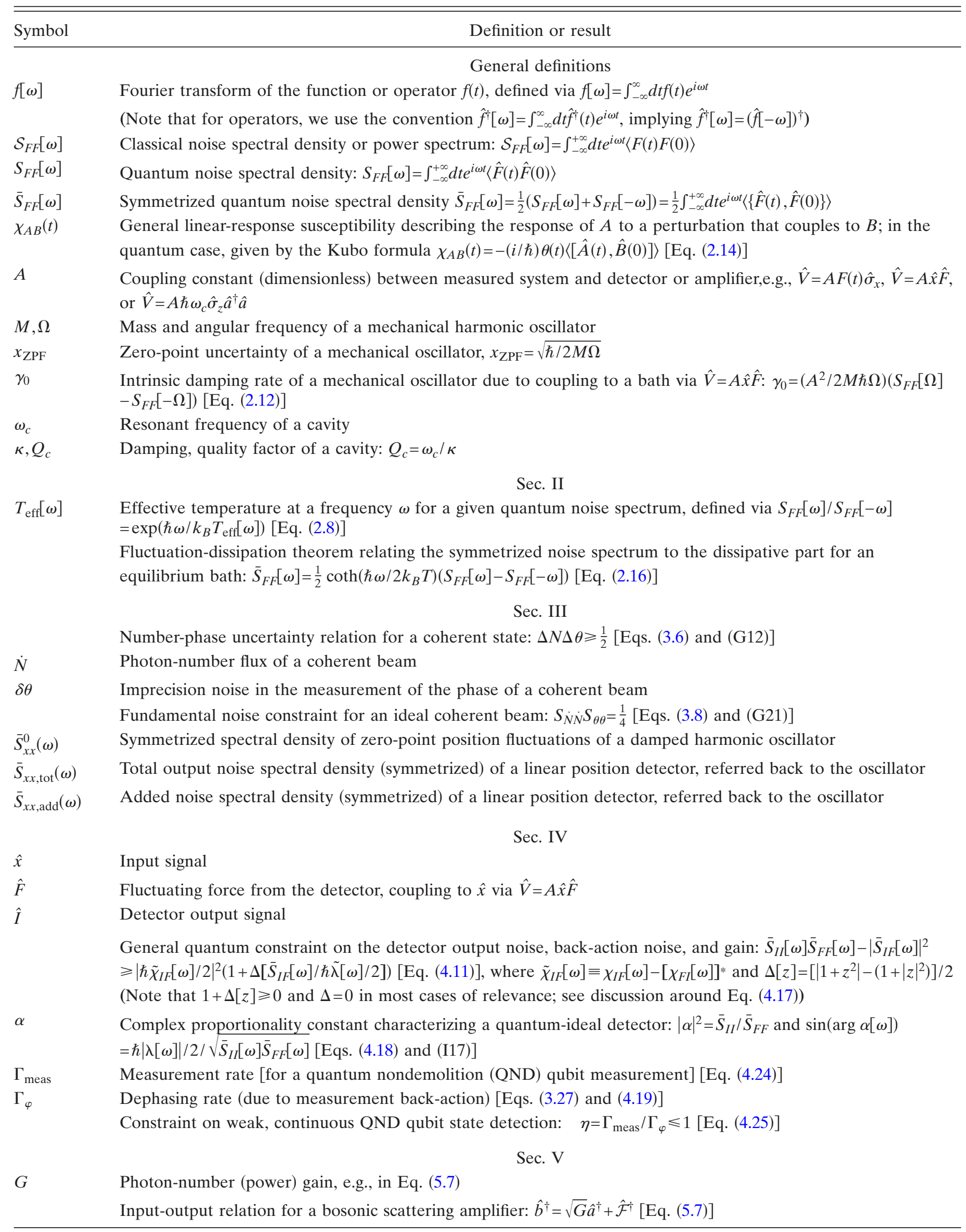


TABLE I. (Continued.)

\begin{tabular}{|c|c|}
\hline Symbol & Definition or result \\
\hline$(\Delta a)^{2}$ & $\begin{array}{l}\text { Symmetrized field operator uncertainty for the scattering description of a bosonic amplifier: }(\Delta a)^{2} \equiv \frac{1}{2}\left\langle\left\{\hat{a}, \hat{a}^{\dagger}\right\}\right\rangle \\
-|\langle a\rangle|^{2} \\
\text { Standard quantum limit for the noise added by a phase-preserving bosonic scattering amplifier in the high-gain } \\
\text { limit, } G \gg 1 \text {, where }\left\langle(\Delta a)^{2}\right\rangle_{\mathrm{ZPF}}=\frac{1}{2}:(\Delta b)^{2} / G \geqslant(\Delta a)^{2}+\frac{1}{2} \text { [Eq. (5.10)] }\end{array}$ \\
\hline$G_{P}[\omega]$ & $\begin{array}{l}\text { Dimensionless power gain of a linear position detector or voltage amplifier(maximum ratio of the power } \\
\text { delivered by the detector output to a load, vs the power fed into signal source): } \\
G_{P}[\omega]=\left|\chi_{I F}[\omega]\right|^{2} /\left(4 \operatorname{Im} \chi_{F F}[\omega] \operatorname{Im} \chi_{I I}[\omega]\right)[\text { Eq. }(5.52)] \\
\text { For a quantum-ideal detector, in the high-gain limit: } G_{P} \simeq\left[(\operatorname{Im} \alpha /|\alpha|)\left(4 k_{B} T_{\text {eff }} / \hbar \omega\right)\right]^{2}[\text { Eq. (5.57)] }\end{array}$ \\
\hline $\bar{S}_{x x, \mathrm{eq}}[\omega, T]$ & Intrinsic equilibrium noise $\bar{S}_{x x, \mathrm{eq}}[\omega, T]=\hbar \operatorname{coth}\left(\hbar \omega / 2 k_{B} T\right)\left(-\operatorname{Im} \chi_{x x}[\omega]\right)[$ Eq. (5.59)] \\
\hline$A_{\text {opt }}$ & $\begin{array}{l}\text { Optimal coupling strength of a linear position detector which minimizes the added noise at frequency } \omega: A_{\mathrm{opt}}^{4}[\omega] \\
=\bar{S}_{I I}[\omega] /\left(\left|\lambda[\omega] \chi_{x x}[\omega]\right|^{2} \bar{S}_{F F}[\omega]\right)[\text { Eq. (5.64)] }\end{array}$ \\
\hline$\gamma\left[A_{\mathrm{opt}}\right]$ & $\begin{array}{l}\text { Detector-induced damping of a quantum-limited linear position detector at optimal coupling; satisfies } \gamma\left[A_{\text {opt }}\right] / \gamma_{0} \\
+\gamma\left[A_{\text {opt }}\right]=|\operatorname{Im} \alpha / \alpha|\left(1 / \sqrt{G_{P}[\Omega]}\right)=\hbar \Omega / 4 k_{B} T_{\text {eff }} \ll 1[\text { Eq. }(5.69)] \\
\text { Standard quantum limit for the added noise spectral density of a linear position detector (valid at each frequency } \\
\omega): S_{x x} \text {,add }[\omega] \geqslant \lim _{T \rightarrow 0} S_{x x \text {,eq }}[w, T][\text { Eq. }(5.62)] \\
\text { Effective increase in oscillator temperature due to coupling to the detector back-action, for an ideal detector, } \\
\text { with } \hbar \Omega / k_{B} \ll T_{\text {bath }} \ll T_{\text {eff }}: \quad T_{\text {osc }} \equiv\left(\gamma T_{\text {eff }}+\gamma_{0} T_{\text {bath }}\right) /\left(\gamma+\gamma_{0}\right) \rightarrow \hbar \Omega / 4 k_{B}+T_{\text {bath }} \text { [Eq. (5.70)] }\end{array}$ \\
\hline$Z_{\text {in }}, Z_{\text {out }}$ & Input and output impedances of a linear voltage amplifier \\
\hline$Z_{s}$ & Impedance of signal source attached to input of a voltage amplifier \\
\hline$\lambda_{V}$ & Voltage gain of a linear voltage amplifier \\
\hline$\tilde{V}(t)$ & $\begin{array}{l}\text { Voltage noise of a linear voltage amplifier (Proportional to the intrinsic output noise of the generic } \\
\text { linear-response detector [Eq. (5.81)]) }\end{array}$ \\
\hline$\tilde{I}(t)$ & $\begin{array}{l}\text { Current noise of a linear voltage amplifier (Related to the back-action force noise of the generic linear-response } \\
\text { detector [Eqs. (5.80)]) }\end{array}$ \\
\hline$T_{N}$ & Noise temperature of an amplifier [defined in Eq. (5.74)] \\
\hline$Z_{N}$ & $\begin{array}{l}\text { Noise impedance of a linear voltage amplifier [Eq. (5.77)] } \\
\text { Standard quantum limit on the noise temperature of a linear voltage amplifier: } k_{B} T_{N}[\omega] \geqslant \hbar \omega / 2[\text { Eq. (5.89)] }\end{array}$ \\
\hline & Sec. VI \\
\hline$\hat{V}_{a}\left(\hat{V}_{b}\right)$ & $\begin{array}{l}\text { Voltage at the input (output) of the amplifier } \\
\text { Relation to bosonic mode operators: Eq. }(6.2 \mathrm{a})\end{array}$ \\
\hline$\hat{I}_{a}\left(\hat{I}_{b}\right)$ & $\begin{array}{l}\text { Current drawn at the input (leaving the output) of the amplifier } \\
\text { Relation to bosonic mode operators: Eq. }(6.2 \mathrm{~b})\end{array}$ \\
\hline$\lambda_{I}^{\prime}$ & Reverse current gain of the amplifier \\
\hline$s[\omega]$ & $\begin{array}{l}\text { Input-output } 2 \times 2 \text { scattering matrix of the amplifier [Eq. (6.3)] } \\
\text { Relation to op-amp parameters } \lambda_{V}, \lambda_{I}^{\prime}, Z_{\text {in }}, Z_{\text {out }}: \text { Eqs. }(6.7)\end{array}$ \\
\hline$\hat{\tilde{V}}(\hat{\tilde{I}})$ & Voltage (current) noise operators of the amplifier \\
\hline$\hat{\mathcal{F}}_{a}[\omega], \hat{\mathcal{F}}_{b}[\omega]$ & $\begin{array}{l}\text { Input (output) port noise operators in the scattering description [Eq. (6.3)] } \\
\text { Relation to op-amp noise operators } \hat{\tilde{V}}, \hat{\tilde{I}} \text { : Eq. (6.9) }\end{array}$ \\
\hline
\end{tabular}

Appendixes. Note that, while some aspects of the topics discussed in this review have been studied in the quantum optics and quantum dissipative systems communities and are the subject of several comprehensive books (Braginsky and Khalili, 1992; Weiss, 1999; Gardiner and Zoller, 2000; Haus, 2000), they are somewhat newer to the condensed matter physics community; moreover, some of the technical machinery developed in these fields is not directly applicable to the study of quantum noise in quantum electronic systems. Finally, note that while this article is a review, there is considerable new material presented, especially in our discussion of quantum amplification (see Secs. V.D and VI).

\section{QUANTUM NOISE SPECTRA}

\section{A. Introduction to quantum noise}

In classical physics, the study of a noisy timedependent quantity invariably involves its spectral density $\mathcal{S}[\omega]$. The spectral density tells us the intensity of the noise at a given frequency and is directly related to the 
TABLE II. Contents of online appendix material. Page numbers refer to the supplementary material.

\begin{tabular}{ll}
\hline \hline Section & Page \\
\hline A. Basics of classical and quantum noise & 1 \\
B. Quantum spectrum analyzers: further details & 4 \\
C. Modes, transmission lines and classical & 8 \\
input-output theory & \\
D. Quantum modes and noise of a transmission line & 15 \\
E. Back-action and input-output theory for driven & 18 \\
damped cavities & \\
F. Information theory and measurement rate & 29 \\
G. Number phase uncertainty & 30 \\
H. Using feedback to reach the quantum limit & 31 \\
I. Additional technical details & 34 \\
\hline \hline
\end{tabular}

autocorrelation function of the noise. ${ }^{2}$ In a similar fashion, the study of quantum noise involves quantum noise spectral densities. These are defined in a manner that mimics the classical case

$$
S_{x x}[\omega]=\int_{-\infty}^{+\infty} d t e^{i \omega t}\langle\hat{x}(t) \hat{x}(0)\rangle .
$$

Here $\hat{x}$ is a quantum operator (in the Heisenberg representation) whose noise we are interested in, and the angular brackets indicate the quantum statistical average evaluated using the quantum density matrix. Note that we use $\mathcal{S}[\omega]$ throughout this review to denote the spectral density of a classical noise, while $S[\omega]$ will denote a quantum noise spectral density.

As a simple introductory example illustrating important differences from the classical limit, consider the position noise of a simple harmonic oscillator having mass $M$ and frequency $\Omega$. The oscillator is maintained in equilibrium with a large heat bath at temperature $T$ via some infinitesimal coupling, which we ignore in considering the dynamics. The solutions of the Heisenberg equations of motion are the same as for the classical case but with the initial position $x$ and momentum $p$ replaced by the corresponding quantum operators. It follows that the position autocorrelation function is

$$
\begin{aligned}
G_{x x}(t)=\langle\hat{x}(t) \hat{x}(0)\rangle= & \langle\hat{x}(0) \hat{x}(0)\rangle \cos (\Omega t) \\
& +\langle\hat{p}(0) \hat{x}(0)\rangle \frac{1}{M \Omega} \sin (\Omega t) .
\end{aligned}
$$

Classically the second term on the right-hand side (RHS) vanishes because in thermal equilibrium $x$ and $p$ are uncorrelated random variables. As we will see shortly for the quantum case, the symmetrized (sometimes called the "classical") correlator vanishes in thermal equilibrium, just as it does classically: $\langle\hat{x} \hat{p}+\hat{p} \hat{x}\rangle=0$.

\footnotetext{
${ }^{2}$ For readers unfamiliar with the basics of classical noise, a compact review is given in Appendix A (supplementary material).
}

Note, however, that in the quantum case the canonical commutation relation between position and momentum implies there must be some correlations between the two, namely, $\langle\hat{x}(0) \hat{p}(0)\rangle-\langle\hat{p}(0) \hat{x}(0)\rangle=i \hbar$. These correlations are easily evaluated by writing $\hat{x}$ and $\hat{p}$ in terms of harmonic oscillator ladder operators. We find that in thermal equilibrium $\langle\hat{p}(0) \hat{x}(0)\rangle=-i \hbar / 2$ and $\langle\hat{x}(0) \hat{p}(0)\rangle$ $=+i \hbar / 2$. Not only are the position and momentum correlated, but their correlator is imaginary: ${ }^{3}$ This means that, despite the fact that the position is Hermitian observable with real eigenvalues, its autocorrelation function is complex and given from Eq. (2.2) by

$$
G_{x x}(t)=x_{\mathrm{ZPF}}^{2}\left\{n_{B}(\hbar \Omega) e^{+i \Omega t}+\left[n_{B}(\hbar \Omega)+1\right] e^{-i \Omega t}\right\},
$$

where $x_{\mathrm{ZPF}}^{2} \equiv \hbar / 2 M \Omega$ is the RMS zero-point uncertainty of $x$ in the quantum ground state and $n_{B}$ is the BoseEinstein occupation factor. The complex nature of the autocorrelation function follows from the fact that the operator $\hat{x}$ does not commute with itself at different times.

Because the correlator is complex it follows that the spectral density is not symmetric in frequency,

$$
\begin{aligned}
S_{x x}[\omega]= & 2 \pi x_{\mathrm{ZPF}}^{2}\left\{n_{B}(\hbar \Omega) \delta(\omega+\Omega)\right. \\
& \left.+\left[n_{B}(\hbar \Omega)+1\right] \delta(\omega-\Omega)\right\} .
\end{aligned}
$$

In contrast, a classical autocorrelation function is always real, and hence a classical noise spectral density is always symmetric in frequency. Note that in the hightemperature limit $k_{B} T \gg \hbar \Omega$ we have $n_{B}(\hbar \Omega) \sim n_{B}(\hbar \Omega)$ $+1 \sim k_{B} T / \hbar \Omega$. Thus, in this limit $S_{x x}[\omega]$ becomes symmetric in frequency as expected classically, and coincides with the classical expression for the position spectral density [cf. Eq. (A12)].

The Bose-Einstein factors suggest a way to understand the frequency asymmetry of Eq. (2.4): the positivefrequency part of the spectral density has to do with stimulated emission of energy into the oscillator and the negative-frequency part of the spectral density has to do with emission of energy by the oscillator. That is, the positive-frequency part of the spectral density is a measure of the ability of the oscillator to absorb energy, while the negative-frequency part is a measure of the ability of the oscillator to emit energy. As we will see, this is generally true, even for nonthermal states. Figure 1 shows this idea for the case of the voltage noise spectral density of a resistor (see Appendix D.3 for more details). Note that the result Eq. (2.4) can be extended to the case of a bath of many harmonic oscillators. As described in Appendix D a resistor can be modeled as an infinite set of harmonic oscillators and from this model the Johnson or Nyquist noise of a resistor can be derived.

\footnotetext{
${ }^{3}$ Notice that this occurs because the product of two noncommuting Hermitian operators is not itself a Hermitian operator.
} 


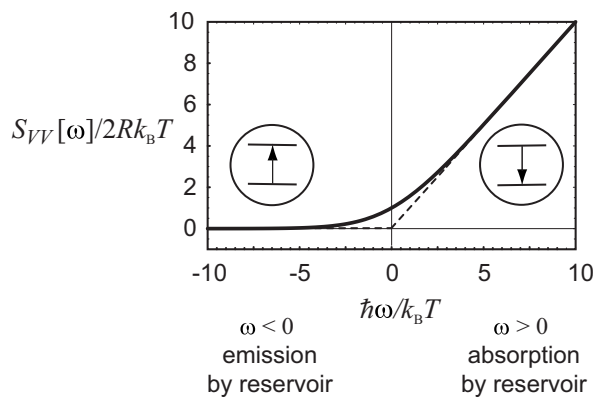

FIG. 1. Quantum noise spectral density of voltage fluctuations across a resistor (resistance $R$ ) as a function of frequency at zero temperature (dashed line) and finite temperature (solid line).

\section{B. Quantum spectrum analyzers}

The qualitative picture described previously can be confirmed by considering simple systems which act as effective spectrum analyzers of quantum noise. The simplest such example is a quantum two-level system (TLS) coupled to a quantum noise source (Aguado and Kouwenhoven, 2000; Gavish et al., 2000; Schoelkopf et al., 2003). With the TLS described as a fictitious spin-1/2 particle with spin down (spin up) representing the ground state (excited state), its Hamiltonian is $\hat{H}_{0}$ $=\left(\hbar \omega_{01} / 2\right) \hat{\sigma}_{z}$, where $\hbar \omega_{01}$ is the energy splitting between the two states. The TLS is then coupled to an external noise source via an additional term in the Hamiltonian,

$$
\hat{V}=A \hat{F} \hat{\sigma}_{x}
$$

where $A$ is a coupling constant and the operator $\hat{F}$ represents the external noise source. The coupling Hamiltonian $\hat{V}$ can lead to the exchange of energy between the two-level system and noise source and hence transitions between its two eigenstates. The corresponding Fermi golden rule transition rates can be compactly expressed in terms of the quantum noise spectral density of $\hat{F}$, $S_{F F}[\omega]$,

$$
\begin{aligned}
& \Gamma_{\uparrow}=\left(A^{2} / \hbar^{2}\right) S_{F F}\left[-\omega_{01}\right], \\
& \Gamma_{\downarrow}=\left(A^{2} / \hbar^{2}\right) S_{F F}\left[+\omega_{01}\right] .
\end{aligned}
$$

Here $\Gamma_{\uparrow}$ is the rate at which the qubit is excited from its ground to excited state and $\Gamma_{\downarrow}$ is the corresponding rate for the opposite relaxation process. As expected, positive- (negative-) frequency noise corresponds to absorption (emission) of energy by the noise source. Note that if the noise source is in thermal equilibrium at temperature $T$, the transition rates of the TLS must satisfy the detailed balance relation $\Gamma_{\uparrow} / \Gamma_{\downarrow}=e^{-\beta \hbar \omega_{01}}$, where $\beta$ $=1 / k_{B} T$. This in turn implies that in thermal equilibrium the quantum noise spectral density must satisfy

$$
S_{F F}\left[+\omega_{01}\right]=e^{\beta \hbar \omega_{01}} S_{F F}\left[-\omega_{01}\right] .
$$

The more general situation is where the noise source is not in thermal equilibrium; in this case, no general de- tailed balance relation holds. However, if we are concerned only with a single particular frequency, then it is always possible to define an "effective temperature" $T_{\text {eff }}$ for the noise using Eq. (2.7), i.e.,

$$
k_{B} T_{\text {eff }}[\omega] \equiv \frac{\hbar \omega}{\log \left[S_{F F}[\omega] / S_{F F}[-\omega]\right]} .
$$

Note that for a nonequilibrium system $T_{\text {eff }}$ will in general be frequency dependent. In NMR language, $T_{\text {eff }}$ will simply be the "spin temperature" of our TLS spectrometer once it reaches steady state after being coupled to the noise source.

Another simple quantum noise spectrometer is a harmonic oscillator (frequency $\Omega$, mass $M$, and position $x$ ) coupled to a noise source [see, e.g., Schwinger (1961) and Dykman (1978)]. The coupling Hamiltonian is now

$$
\hat{V}=A \hat{x} \hat{F}=A\left[x_{\mathrm{ZPF}}\left(\hat{a}+\hat{a}^{\dagger}\right)\right] \hat{F}
$$

where $\hat{a}$ is the oscillator annihilation operator, $\hat{F}$ is the operator describing the fluctuating noise, and $A$ is again a coupling constant. We see that $-A \hat{F}$ plays the role of a fluctuating force acting on the oscillator. In complete analogy to the previous section, noise in $\hat{F}$ at the oscillator frequency $\Omega$ can cause transitions between the oscillator energy eigenstates. The corresponding Fermi golden rule transition rates are again simply related to the noise spectrum $S_{F F}[\omega]$. Incorporating these rates into a simple master equation describing the probability to find the oscillator in a particular energy state, one finds that the stationary state of the oscillator is a BoseEinstein distribution evaluated at the effective temperature $T_{\text {eff }}[\Omega]$ defined in Eq. (2.8). Further, one can use the master equation to derive a classical-looking equation for the average energy $\langle E\rangle$ of the oscillator (see Appendix B.2),

$$
d\langle E\rangle / d t=P-\gamma\langle E\rangle,
$$

where

$$
\begin{aligned}
& P=\left(A^{2} / 4 M\right)\left(S_{F F}[\Omega]+S_{F F}[-\Omega]\right) \equiv A^{2} \bar{S}_{F F}[\Omega] / 2 M, \\
& \gamma=\left(A^{2} x_{\mathrm{ZPF}}^{2} / \hbar^{2}\right)\left(S_{F F}[\Omega]-S_{F F}[-\Omega]\right) .
\end{aligned}
$$

The two terms in Eq. (2.10) describe, heating and damping of the oscillator by the noise source, respectively. The heating effect of the noise is completely analogous to what happens classically: a random force causes the oscillator's momentum to diffuse, which in turn causes $\langle E\rangle$ to grow linearly in time at rate proportional to the force noise spectral density. In the quantum case, Eq. (2.11) indicates that it is the symmetric-in-frequency part of the noise spectrum, $\bar{S}_{F F}[\Omega]$, which is responsible for this effect, and which thus plays the role of a classical noise source. This is another reason why $\bar{S}_{F F}[\omega]$ is often 
referred to as the "classical" part of the noise. ${ }^{4}$ In contrast, we see that the asymmetric-in-frequency part of the noise spectrum is responsible for the damping. This also has a simple heuristic interpretation: damping is caused by the net tendency of the noise source to absorb, rather than emit, energy from the oscillator.

The damping induced by the noise source may equivalently be attributed to the oscillator's motion inducing an average value to $\langle F\rangle$ which is out of phase with $x$, i.e., $\delta\langle A F(t)\rangle=-M \gamma \dot{x}(t)$. Standard quantum linear-response theory yields

$$
\delta\langle A \hat{F}(t)\rangle=A^{2} \int d t^{\prime} \chi_{F F}\left(t-t^{\prime}\right)\left\langle\hat{x}\left(t^{\prime}\right)\right\rangle
$$

where we have introduced the susceptibility

$$
\chi_{F F}(t)=(-i / \hbar) \theta(t)\langle[\hat{F}(t), \hat{F}(0)]\rangle .
$$

Using the fact that the oscillator's motion involves only the frequency $\Omega$, we thus have

$$
\gamma=\left(2 A^{2} x_{\mathrm{ZPF}}^{2} / \hbar\right)\left[-\operatorname{Im} \chi_{F F}[\Omega]\right] .
$$

A straightforward manipulation of Eq. (2.14) for $\chi_{F F}$ shows that this expression for $\gamma$ is exactly equivalent to our previous expression, Eq. (2.12).

In addition to giving insight on the meaning of the symmetric and asymmetric parts of a quantum noise spectral density, the above example also directly yields the quantum version of the fluctuation-dissipation theorem (Callen and Welton, 1951). As we saw earlier, if our noise source is in thermal equilibrium, the positive- and negative-frequency parts of the noise spectrum are strictly related to one another by the condition of detailed balance [cf. Eq. (2.7)]. This in turn lets us link the classical symmetric-in-frequency part of the noise to the damping (i.e., the asymmetric-in-frequency part of the noise). Setting $\beta=1 / k_{B} T$ and making use of Eq. (2.7), we have

$$
\begin{aligned}
\bar{S}_{F F}[\Omega] & \equiv \frac{S_{F F}[\Omega]+S_{F F}[-\Omega]}{2} \\
& =\frac{1}{2} \operatorname{coth}(\beta \hbar \Omega / 2)\left(S_{F F}[\Omega]-S_{F F}[-\Omega]\right) \\
& =\operatorname{coth}(\beta \hbar \Omega / 2) \frac{\hbar \Omega M}{A^{2}} \gamma[\Omega] .
\end{aligned}
$$

Thus, in equilibrium, the condition that noise-induced transitions obey detailed balance immediately implies that noise and damping are related to one another via the temperature. For $T \gg \hbar \Omega$, we recover the more familiar classical version of the fluctuation-dissipation theorem

\footnotetext{
${ }^{4}$ Note that with our definition $\left\langle\hat{F}^{2}\right\rangle=\int_{-\infty}^{\infty}(d \omega / 2 \pi) \bar{S}_{F F}[\omega]$. It is common in engineering contexts to define so-called one-sided classical spectral densities, which are equal to twice our definition.
}

$$
A^{2} \bar{S}_{F F}[\Omega]=2 k_{B} T M \gamma .
$$

Further insight into the fluctuation-dissipation theorem is provided in Appendix C.3, where we discuss it in the simple but instructive context of a transmission line terminated by an impedance $Z[\omega]$.

We have thus considered two simple examples of methods of measure quantum noise spectral densities. Further details, as well as examples of other quantum noise spectrum analyzers, are given in Appendix B.

\section{QUANTUM MEASUREMENTS}

Having introduced both quantum noise and quantum spectrum analyzers, we are now in a position to introduce the general topic of quantum measurements. All practical measurements are affected by noise. Certain quantum measurements remain limited by quantum noise even though they use completely ideal apparatus. As we will see, the limiting noise here is associated with the fact that canonically conjugate variables are incompatible observables in quantum mechanics.

The simplest idealized description of a quantum measurement, introduced by von Neumann (von Neumann, 1932; Wheeler and Zurek, 1984; Bohm, 1989; Haroche and Raimond, 2006), postulates that the measurement process instantaneously collapses the system's quantum state onto one of the eigenstates of the observable to be measured. As a consequence, any initial superposition of these eigenstates is destroyed and the values of observables conjugate to the measured observable are perturbed. This perturbation is an intrinsic feature of quantum mechanics and cannot be avoided in any measurement scheme, be it of the projection type described by von Neumann or a rather weak continuous measurement to be analyzed further below.

To form a more concrete picture of quantum measurement, we begin by noting that every quantum measurement apparatus consists of a macroscopic "pointer" coupled to the microscopic system to be measured. [A specific model is discussed by Allahverdyan et al. (2001).] This pointer is sufficiently macroscopic that its position can be read out "classically." The interaction between the microscopic system and the pointer is arranged so that the two become strongly correlated. One of the simplest possible examples of a quantum measurement is that of the Stern-Gerlach apparatus, which measures the projection of the spin of an $S=1 / 2$ atom along some chosen direction. What is really measured in the experiment is the final position of the atom on the detector plate. However, the magnetic field gradient in the magnet causes this position to be perfectly correlated ("entangled") with the spin projection so that the latter can be inferred from the former. Suppose, for example that the initial state of the atom is the product of a spatial wave function $\xi_{0}(\vec{r})$ centered on the entrance to the magnet and a spin state that is the superposition of up and down spins corresponding to the eigenstate of $\hat{\sigma}_{x}$, 


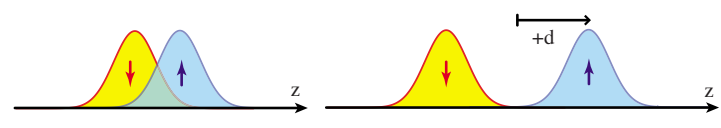

FIG. 2. (Color online) Schematic of position distributions of an atom in the detector plane of a Stern-Gerlach apparatus whose field gradient is in the $z$ direction. For small values of the displacement $d$ (described in the text), there is significant overlap of the distributions and the spin cannot be unambiguously inferred from the position. For large values of $d$, the spin is perfectly entangled with position and can be inferred from the position. This is the limit of strong projective measurement.

$$
\left|\Psi_{0}\right\rangle=(1 / \sqrt{2})\{|\uparrow\rangle+|\downarrow\rangle\}\left|\xi_{0}\right\rangle .
$$

After passing through a magnet with field gradient in the $z$ direction, an atom with spin up is deflected upward and an atom with spin down is deflected downward. By the linearity of quantum mechanics, an atom in a spin superposition state thus ends up in a superposition of the form

$$
\left|\Psi_{1}\right\rangle=(1 / \sqrt{2})\left\{|\uparrow\rangle\left|\xi_{+}\right\rangle+|\downarrow\rangle\left|\xi_{-}\right\rangle\right\},
$$

where $\left\langle\vec{r} \mid \xi_{ \pm}\right\rangle=\psi_{1}(\vec{r} \pm d \hat{z})$ are spatial orbitals peaked in the plane of the detector. The deflection $d$ is determined by the device geometry and the magnetic field gradient. The $z$-direction position distribution of the particle for each spin component is shown in Fig. 2. If $d$ is sufficiently large compared to the wave packet spread then, given the position of the particle, one can unambiguously determine the distribution from which it came and hence the value of the spin projection of the atom. This is the limit of a strong "projective" measurement.

In the initial state one has $\left\langle\Psi_{0}\left|\hat{\sigma}_{x}\right| \Psi_{0}\right\rangle=+1$, but in the final state one has

$$
\left\langle\Psi_{1}\left|\hat{\sigma}_{x}\right| \Psi_{1}\right\rangle=\frac{1}{2}\left\{\left\langle\xi_{-} \mid \xi_{+}\right\rangle+\left\langle\xi_{+} \mid \xi_{-}\right\rangle\right\} .
$$

For sufficiently large $d$ the states $\xi_{ \pm}$are orthogonal and thus the act of $\hat{\sigma}_{z}$ measurement destroys the spin coherence

$$
\left\langle\Psi_{1}\left|\hat{\sigma}_{x}\right| \Psi_{1}\right\rangle \rightarrow 0 .
$$

This is what we mean by projection or wave function "collapse." The result of measurement of the atom position will yield a random and unpredictable value of $\pm \frac{1}{2}$ for the $z$ projection of the spin. This destruction of the coherence in the transverse spin components by a strong measurement of the longitudinal spin component is the first of many examples we will see of the Heisenberg uncertainty principle in action. Measurement of one variable destroys information about its conjugate variable. We study several examples in which we understand microscopically how it is that the coupling to the measurement apparatus causes the back-action quantum noise which destroys our knowledge of the conjugate variable.

In the special case where the eigenstates of the observable we are measuring are also stationary states (i.e., energy eigenstates), a second measurement of the observable would reproduce exactly the same result, thus providing a way to confirm the accuracy of the measurement scheme. These optimal kinds of repeatable measurements are called quantum nondemolition (QND) measurements (Braginsky et al., 1980; Braginsky and Khalili, 1992, 1996; Peres, 1993). A simple example would be a sequential pair of Stern-Gerlach devices oriented in the same direction. In the absence of stray magnetic perturbations, the second apparatus would always yield the same answer as the first. In practice, one terms a measurement QND if the observable being measured is an eigenstate of the ideal Hamiltonian of the measured system (i.e., one ignores any couplings between this system and sources of dissipation). This is reasonable if such couplings give rise to dynamics on time scales longer than needed to complete the measurement. This point is elaborated in Sec. VII, where we discuss practical considerations related to the quantum limit. We also discuss in that section the fact that the repeatability of QND measurements is of fundamental practical importance in overcoming detector inefficiencies (Gambetta et al., 2007).

A common confusion is to think that a QND measurement has no effect on the state of the system being measured. While this is true if the initial state is an eigenstate of the observable, it is not true in general. Consider again our example of a spin oriented in the $x$ direction. The result of the first $\hat{\sigma}_{z}$ measurement will be that the state randomly and completely unpredictably collapses onto one of the two $\hat{\sigma}_{z}$ eigenstates: the state is indeed altered by the measurement. However, all subsequent measurements using the same orientation for the detectors will always agree with the result of the first measurement. Thus QND measurements may affect the state of the system, but never the value of the observable (once it is determined). Other examples of QND measurements include (i) measurement of the electromagnetic field energy stored inside a cavity by determining the radiation pressure exerted on a moving piston (Braginsky and Khalili, 1992), (ii) detection of the presence of a photon in a cavity by its effect on the phase of an atom's superposition state (Nogues et al., 1999; Haroche and Raimond, 2006), and (iii) the "dispersive" measurement of a qubit state by its effect on the frequency of a driven microwave resonator (Blais et al., 2004; Wallraff et al., 2004; Lupaşcu et al., 2007), which is the first canonical example described below.

In contrast to the above, in non-QND measurements the back-action of the measurement will affect the observable being studied. The canonical example we consider is the position measurement of a harmonic oscillator. Since the position operator does not commute with the Hamiltonian, the QND criterion is not satisfied. Other examples of non-QND measurements include (i) photon counting via photodetectors that absorb the photons, (ii) continuous measurements where the observable does not commute with the Hamiltonian, thus inducing a time dependence of the measurement result, and (iii) measurements that can be repeated only after a time longer than the energy relaxation time of the system (e.g., for a qubit, $T_{1}$ ). 


\section{A. Weak continuous measurements}

In discussing "real" quantum measurements, another key notion to introduce is that of weak continuous measurements (Braginsky and Khalili, 1992). Many measurements in practice take an extended time interval to complete, which is much longer than the "microscopic" time scales (oscillation periods, etc.) of the system. The reason may be quite simply that the coupling strength between the detector and the system cannot be made arbitrarily large, and one has to wait for the effect of the system on the detector to accumulate. For example, in our Stern-Gerlach measurement, suppose that we are only able to achieve small magnetic field gradients and that, consequently, the displacement $d$ cannot be made large compared to the wave packet spread (see Fig. 2). In this case the states $\xi_{ \pm}$would have nonzero overlap and it would not be possible to reliably distinguish them: we thus would have only a weak measurement. However, by cascading together a series of such measurements and taking advantage of the fact that they are QND, we can eventually achieve an unambiguous strong projective measurement: at the end of the cascade, we are certain of which $\hat{\sigma}_{z}$ eigenstate the spin is in. During this process, the overlap of $\xi_{ \pm}$would gradually fall to zero corresponding to a smooth continuous loss of phase coherence in the transverse spin components. At the end of the process, the QND nature of the measurement ensures that the probability of measuring $\sigma_{z}=\uparrow$ or $\downarrow$ will accurately reflect the initial wave function. Note that it is only in this case of weak continuous measurements that it makes sense to define a measurement rate in terms of a rate of gain of information about the variable being measured, and a corresponding dephasing rate, the rate at which information about the conjugate variable is being lost. We see that these rates are intimately related via the Heisenberg uncertainty principle.

While strong projective measurements may seem to be the ideal, there are many cases where one may intentionally desire a weak continuous measurement; as discussed in the Introduction. There are many practical examples of weak continuous measurement schemes. These include (i) charge measurements, where the current through a device [e.g., quantum point contact (QPC) or single-electron transistor (SET)] is modulated by the presence or absence of a nearby charge, and where it is necessary to wait for a sufficiently long time to overcome the shot noise and distinguish between the two current values, (ii) the weak dispersive qubit measurement discussed below, and (iii) displacement detection of a nanomechanical beam (e.g., optically or by capacitive coupling to a charge sensor), where one looks at the two quadrature amplitudes of the signal produced at the beam's resonance frequency.

Not surprisingly, quantum noise plays a crucial role in determining the properties of a weak continuous quantum measurement. For such measurements, noise both determines the back-action effect of the measurement on the measured system and how quickly information is acquired in the measurement process. Previously, we saw that a crucial feature of quantum noise is the asymmetry between positive and negative frequencies; we further saw that this corresponds to the difference between absorption and emission events. For measurements, another key aspect of quantum noise will be important: as will be discussed extensively, quantum mechanics places constraints on the noise of any system capable of acting as a detector or amplifier. These constraints in turn place restrictions on any weak continuous measurement, and lead directly to quantum limits on how well one can make such a measurement.

In the remainder of this section, we give an introduction to how one describes a weak continuous quantum measurement, considering the specific examples of using parametric coupling to a resonant cavity for QND detection of the state of a qubit and the (necessarily nonQND) detection of the position of a harmonic oscillator. In the following section (Sec. IV), we give a derivation of a very general quantum-mechanical constraint on the noise of any system capable of acting as a detector, and show how this constraint directly leads to the quantum limit on qubit detection. Finally, in Sec. V, we turn to the important but slightly more involved case of a quantum linear amplifier or position detector. We show that the basic quantum noise constraint derived Sec. IV again leads to a quantum limit; here this limit is on how small one can make the added noise of a linear amplifier.

Before leaving this section, it is worth pointing out that the theory of weak continuous measurements is sometimes described in terms of some set of auxiliary systems which are sequentially and momentarily weakly coupled to the system being measured (see Appendix E). One then envisions a sequence of projective von Neumann measurements on the auxiliary variables. The weak entanglement between the system of interest and one of the auxiliary variables leads to a kind of partial collapse of the system wave function (more precisely, the density matrix), which is described in mathematical terms not by projection operators, but rather by positive operator valued measures (POVMs). We will not use this and the related "quantum trajectory" language here, but direct the interested reader to the literature for more information on this important approach (Peres, 1993; Brun, 2002; Haroche and Raimond, 2006; Jordan and Korotkov, 2006).

\section{B. Measurement with a parametrically coupled resonant cavity}

A simple yet experimentally practical example of a quantum detector consists of a resonant optical or $\mathrm{rf}$ cavity parametrically coupled to the system being measured. Changes in the variable being measured (e.g., the state of a qubit or the position of an oscillator) shift the cavity frequency and produce a varying phase shift in the carrier signal reflected from the cavity. This changing phase shift can be converted (via homodyne interferometry) into a changing intensity; this can then be detected using diodes or photomultipliers. 
In this section, we analyze weak continuous measurements made using such a parametric cavity detector; this will serve as a good introduction to the more general approaches presented later. We show that this detector is capable of reaching the "quantum limit" meaning that it can be used to make a weak continuous measurement, as optimally as is allowed by quantum mechanics. This is true for both the (QND) measurement of the state of a qubit and the (non-QND) measurement of the position of a harmonic oscillator. Complementary analyses of weak continuous qubit measurement are given by Makhlin et al. (2000, 2001) (using a single-electron transistor) and by Gurvitz (1997), Korotkov (2001b), Korotkov and Averin (2001), Pilgram and Büttiker (2002), and Clerk et al. (2003) (using a quantum point contact). We focus here on a high- $Q$ cavity detector; weak qubit measurement with a low- $Q$ cavity was studied by Johansson et al. (2006).

It is worth noting the widespread usage of cavity detectors in experiment. One important current realization is a microwave cavity used to read out the state of a superconducting qubit (Il'ichev et al., 2003; Blais et al., 2004; Izmalkov et al., 2004; Lupaşcu et al., 2004, 2005; Wallraff et al., 2004; Duty et al., 2005; Schuster et al., 2005; Sillanpää et al., 2005). Another class of examples are optical cavities used to measure mechanical degree of freedom. Examples of such systems include those where one of the cavity mirrors is mounted on a cantilever (Arcizet et al., 2006; Gigan et al., 2006; Kleckner and Bouwmeester, 2006). Related systems involve a freely suspended mass (Abramovici et al., 1992; Corbitt et al., 2007), an optical cavity with a thin transparent membrane in the middle (Thompson et al., 2008), and, more generally, an elastically deformable whispering gallery mode resonator (Schliesser et al., 2006). Systems where a microwave cavity is coupled to a mechanical element are also under active study (Blencowe and Buks, 2007; Regal et al., 2008; Teufel et al., 2008).

We start our discussion with a general observation. The cavity uses interference and the wave nature of light to convert the input signal to a phase-shifted wave. For small phase shifts we have a weak continuous measurement. Interestingly, it is the complementary particle nature of light that turns out to limit the measurement. As we will see, it both limits the rate at which we can make a measurement (via photon shot noise in the output beam) and also controls the back-action disturbance of the system being measured (due to photon shot noise inside the cavity acting on the system being measured). These dual aspects are an important part of any weak continuous quantum measurement; hence, an understanding of both the output noise (i.e., the measurement imprecision) and back-action noise of detectors will be crucial.

All of our discussion of noise in the cavity system will be framed in terms of the number-phase uncertainty relation for coherent states. A coherent photon state contains a Poisson distribution of the number of photons, implying that the fluctuations in photon number obey $(\Delta N)^{2}=\bar{N}$, where $\bar{N}$ is the mean number of photons. Fur- ther, coherent states are overcomplete and states of different phase are not orthogonal to each other; this directly implies (see Appendix G) that there is an uncertainty in any measurement of the phase. For large $\bar{N}$, this is given by

$$
(\Delta \theta)^{2}=1 / 4 \bar{N} .
$$

Thus, large- $\bar{N}$ coherent states obey the number-phase uncertainty relation

$$
\Delta N \Delta \theta=\frac{1}{2},
$$

analogous to the position-momentum uncertainty relation.

Equation (3.6) can also be usefully formulated in terms of noise spectral densities associated with the measurement. Consider a continuous photon beam carrying an average photon flux $\overline{\dot{N}}$. The variance in the number of photons detected grows linearly in time and can be represented as $(\Delta N)^{2}=S_{N N} t$, where $S_{N N}$ is the white-noise spectral density of photon flux fluctuations. On a physical level, it describes photon shot noise, and is given by $S_{\dot{N} \dot{N}}=\overline{\dot{N}}$.

Consider now the phase of the beam. Any homodyne measurement of this phase will be subject to the same photon shot noise fluctuations discussed above (see Appendix $G$ for more details). Thus, if the phase of the beam has some nominal small value $\theta_{0}$, the output signal from the homodyne detector integrated up to time $t$ will be of the form $I=\theta_{0} t+\int_{0}^{t} d \tau \delta \theta(\tau)$, where $\delta \theta$ is a noise representing the imprecision in our measurement of $\theta_{0}$ due to the photon shot noise in the output of the homodyne detector. An unbiased estimate of the phase obtained from $I$ is $\theta=I / t$, which obeys $\langle\theta\rangle=\theta_{0}$. Further, one has $(\Delta \theta)^{2}=S_{\theta \theta} / t$, where $S_{\theta \theta}$ is the spectral density of the $\delta \theta$ white noise. Comparison with Eq. (3.5) yields

$$
S_{\theta \theta}=1 / 4 \overline{\dot{N}} .
$$

The above results lead us to the fundamental waveparticle relation for ideal coherent beams,

$$
\sqrt{S_{\dot{N} N} S_{\theta \theta}}=\frac{1}{2} \text {. }
$$

Before we study the role that these uncertainty relations play in measurements with high- $Q$ cavities, consider the simplest case of reflection of light from a mirror without a cavity. The phase shift of the beam (having wave vector $k$ ) when the mirror moves a distance $x$ is $2 k x$. Thus, the uncertainty in the phase measurement corresponds to a position imprecision which can again be represented in terms of a noise spectral density $S_{x x}^{\mathrm{I}}$ $=S_{\theta \theta} / 4 k^{2}$. Here the superscript I refers to the fact that this is noise representing imprecision in the measurement, not actual fluctuations in the position. We also need to worry about back-action: each photon hitting the mirror transfers a momentum $2 \hbar k$ to the mirror, so photon shot noise corresponds to a random back-action force noise spectral density $S_{F F}=4 \hbar^{2} k^{2} S_{N N}$. Multiplying 
these together, we have the central result for the product of the back-action force noise and the imprecision,

$$
S_{F F} S_{x x}^{\mathrm{I}}=\hbar^{2} S_{N N} S_{\theta \theta}=\hbar^{2} / 4
$$

or in analogy with Eq. (3.6)

$$
\sqrt{S_{F F} S_{x x}^{\mathrm{I}}}=\hbar / 2 \text {. }
$$

Not surprisingly, the situation considered here is as ideal as possible. Thus, the RHS above is actually a lower bound on the product of imprecision and back-action noise for any detector capable of significant amplification; we will prove this rigorously in Sec. IV.A Equation (3.10) thus represents the quantum limit on the noise of our detector. As we will see shortly, having a detector with quantum-limited noise is a prerequisite for reaching the quantum limit on various different measurement tasks (e.g., continuous position detection of an oscillator and QND qubit state detection). Note that in general, a given detector will have more noise than the quantumlimited value; we devote considerable effort later to determining the conditions needed to achieve the lower bound of Eq. (3.10).

We now turn to the story of measurement using a high- $Q$ cavity; it will be similar to the above discussion, except that we have to account for the filtering of the noise by the cavity response. We relegate relevant calculational details to Appendix E. The cavity is simply described as a single bosonic mode coupled weakly to electromagnetic modes outside the cavity. The Hamiltonian of the system is given by

$$
\hat{H}=H_{0}+\hbar \omega_{c}(1+A \hat{z}) \hat{a}^{\dagger} \hat{a}+\hat{H}_{\text {envt }} .
$$

Here $H_{0}$ is the unperturbed Hamiltonian of the system whose variable $\hat{z}$ (which is not necessarily a position) is being measured, $\hat{a}$ is the annihilation operator for the cavity mode, and $\omega_{c}$ is the cavity resonance frequency in the absence of the coupling $A$. We will take both $A$ and $\hat{z}$ to be dimensionless. The term $\hat{H}_{\text {envt }}$ describes the electromagnetic modes outside the cavity, and their coupling to the cavity; it is responsible for both driving and damping the cavity mode. The damping is parametrized by rate $\kappa$, which tells us how quickly energy leaks out of the cavity; we consider the case of a high-quality factor cavity, where $Q_{c} \equiv \omega_{c} / \kappa \gg 1$.

Turning to the interaction term in Eq. (3.11), we see that the parametric coupling strength $A$ determines the change in frequency of the cavity as the system variable $\hat{z}$ changes. We assume for simplicity that the dynamics of $\hat{z}$ is slow compared to $\kappa$. In this limit the reflected phase shift simply varies slowly in time adiabatically following the instantaneous value of $\hat{z}$. We also assume that the coupling $A$ is small enough that the phase shifts are always very small and hence the measurement is weak. Many photons will have to pass through the cavity before much information is gained about the value of the phase shift and hence the value of $\hat{z}$.

We first consider the case of a one-sided cavity where only one of the mirrors is semitransparent, the other being perfectly reflecting. In this case, a wave incident on the cavity [say, in a one-dimensional (1D) waveguide] will be perfectly reflected, but with a phase shift $\theta$ determined by the cavity and the value of $\hat{z}$. The reflection coefficient at the bare cavity frequency $\omega_{c}$ is simply given by (Walls and Milburn, 1994)

$$
r=-\left(1+2 i A Q_{c} \hat{z}\right) /\left(1-2 i A Q_{c} \hat{z}\right) .
$$

Note that $r$ has unit magnitude because all incident photons are reflected if the cavity is lossless. For weak coupling we can write the reflection phase shift as $r=-e^{i \theta}$, where

$$
\theta \approx 4 Q_{c} A \hat{z}=\left(A \omega_{c} \hat{z}\right) t_{\mathrm{WD}} .
$$

We see that the scattering phase shift is simply the frequency shift caused by the parametric coupling multiplied by the Wigner delay time (Wigner, 1955)

$$
t_{\mathrm{WD}}=\operatorname{Im} \partial \ln r / \partial \omega=4 / \kappa .
$$

Thus the measurement-imprecision noise power for a given photon flux $\overline{\dot{N}}$ incident on the cavity is given by

$$
S_{z z}^{\mathrm{I}}=S_{\theta \theta} /\left(A \omega_{c} t_{\mathrm{WD}}\right)^{2} .
$$

The random part of the generalized back-action force conjugate to $\hat{z}$ is, from Eq. (3.11),

$$
\hat{F}_{z} \equiv-\partial \hat{H} / \partial \hat{z}=-A \hbar \omega_{c} \delta \hat{n},
$$

where, since $\hat{z}$ is dimensionless, $\hat{F}_{z}$ has units of energy. Here $\delta \hat{n}=\hat{n}-\bar{n}=\hat{a}^{\dagger} \hat{a}-\left\langle\hat{a}^{\dagger} \hat{a}\right\rangle$ represents the photonnumber fluctuations around the mean $\bar{n}$ inside the cavity. The back-action force noise spectral density is thus

$$
S_{F_{z} F_{z}}=\left(A \hbar \omega_{c}\right)^{2} S_{n n} .
$$

As shown in Appendix E, the cavity filters the photon shot noise so that at low frequencies $\omega \ll \kappa$, the number fluctuation spectral density is simply

$$
S_{n n}=\bar{n} t_{\mathrm{WD}} .
$$

The mean photon number in the cavity is found to be $\bar{n}=\overline{\dot{N}} t_{\mathrm{WD}}$, where again $\overline{\dot{N}}$ is the mean photon flux incident on the cavity. From this it follows that

$$
S_{F_{z} F_{z}}=\left(A \hbar \omega_{c} t_{\mathrm{WD}}\right)^{2} S_{N N} .
$$

Combining this with Eq. (3.15) again yields the same result as Eq. (3.10) obtained without the cavity. The parametric cavity detector (used in this way) is thus a quantum-limited detector, meaning that the product of its noise spectral densities achieves the ideal minimum value.

We now examine how the quantum limit on the noise of our detector directly leads to quantum limits on different measurement tasks. In particular, we consider the cases of continuous position detection and QND qubit state measurement. 


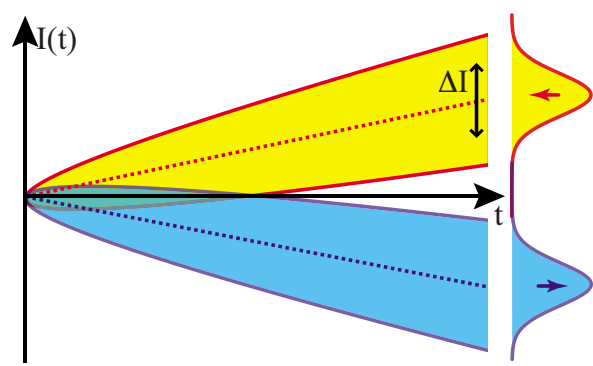

FIG. 3. (Color online) Distribution of the integrated output for the cavity detector $I(t)$ for the two different qubit states. The separation of the means of the distributions grows linearly in time, while the widths of the distributions grow only as $\sqrt{t}$.

\section{QND measurement of the state of a qubit using a resonant cavity}

Here we specialize to the case where the system operator $\hat{z}=\hat{\sigma}_{z}$ represents the state of a spin-1/2 quantum bit. Equation (3.11) becomes

$$
\hat{H}=\frac{1}{2} \hbar \omega_{01} \hat{\sigma}_{z}+\hbar \omega_{c}\left(1+A \hat{\sigma}_{z}\right) \hat{a}^{\dagger} \hat{a}+\hat{H}_{\mathrm{envt}} .
$$

We see that $\hat{\sigma}_{z}$ commutes with all terms in the Hamiltonian and is thus a constant of the motion (assuming that $\hat{H}_{\text {envt }}$ contains no qubit decay terms so that $T_{1}=\infty$ ) and hence the measurement will be QND. From Eq. (3.13) we see that the two states of the qubit produce phase shifts $\pm \theta_{0}$ where

$$
\theta_{0}=A \omega_{c} t_{\mathrm{WD}}
$$

As $\theta_{0} \ll 1$, we need many reflected photons before we are able to determine the state of the qubit. This is a direct consequence of the unavoidable photon shot noise in the output of the detector, and is a basic feature of weak measurements-information on the input is acquired only gradually in time.

Let $I(t)$ be the homodyne signal for the wave reflected from the cavity integrated up to time $t$. Depending on the state of the qubit the mean value of $I$ will be $\langle I\rangle$ $= \pm \theta_{0} t$, and the rms Gaussian fluctuations about the mean will be $\Delta I=\sqrt{S_{\theta \theta} t}$. As shown in Fig. 3 and discussed by in Makhlin et al. (2001), the integrated signal is drawn from one of two Gaussian distributions, which are better and better resolved with increasing time (as long as the measurement is QND). The state of the qubit thus becomes ever more reliably determined. The signal energy to noise energy ratio becomes

$$
\mathrm{SNR}=\langle I\rangle^{2} /(\Delta I)^{2}=\theta_{0}^{2} / S_{\theta \theta} t,
$$

which can be used to define the measurement rate via

$$
\Gamma_{\text {meas }} \equiv \mathrm{SNR} / 2=\theta_{0}^{2} / 2 S_{\theta \theta}=1 / 2 S_{z z}^{\mathrm{I}} .
$$

There is a certain arbitrariness in the scale factor of 2 appearing in the definition of the measurement rate; this particular choice is motivated by precise informationtheoretic grounds (as defined, $\Gamma_{\text {meas }}$ is the rate at which the "accessible information" grows, Appendix F).
While Eq. (3.20) makes it clear that the state of the qubit modulates the cavity frequency, we can easily rewrite this equation to show that this same interaction term is also responsible for the back-action of the measurement (i.e., the disturbance of the qubit state by the measurement process)

$$
\hat{H}=(\hbar / 2)\left(\omega_{01}+2 A \omega_{c} \hat{a}^{\dagger} \hat{a}\right) \hat{\sigma}_{z}+\hbar \omega_{c} \hat{a}^{\dagger} \hat{a}+\hat{H}_{\mathrm{envt}} .
$$

We now see that the interaction can also be viewed as providing a "light shift" (i.e., ac Stark shift) of the qubit splitting frequency (Blais et al., 2004; Schuster et al., $2005)$ which contains a constant part $2 A \bar{n} A \omega_{c}$ plus a randomly fluctuating part $\Delta \omega_{01}=2 \hat{F}_{z} / \hbar$, that depends on $\hat{n}$ $=\hat{a}^{\dagger} \hat{a}$, the number of photons in the cavity. During a measurement, $\hat{n}$ will fluctuate around its mean and act as a fluctuating back-action "force" on the qubit. In the present QND case, noise in $\hat{n}=\hat{a}^{\dagger} \hat{a}$ cannot cause transitions between the two qubit eigenstates. This is the opposite of the situation considered in Sec. II.B, where we wanted to use the qubit as a spectrometer. Despite the lack of any noise-induced transitions, there still is a back-action here, as noise in $\hat{n}$ causes the effective splitting frequency of the qubit to fluctuate in time. For weak coupling, the resulting phase diffusion leads to measurement-induced dephasing of superpositions in the qubit (Blais et al., 2004; Schuster et al., 2005) according to

$$
\left\langle e^{-i \varphi}\right\rangle=\left\langle\exp \left(-i \int_{0}^{t} d \tau \Delta \omega_{01}(\tau)\right)\right\rangle .
$$

For weak coupling the dephasing rate is slow and thus we are interested in long times $t$. In this limit the integral is a sum of a large number of statistically independent terms and thus we can take the accumulated phase to be Gaussian distributed. Using the cumulant expansion we then obtain

$$
\begin{aligned}
\left\langle e^{-i \varphi}\right\rangle & =\exp \left(-\frac{1}{2}\left\langle\left[\int_{0}^{t} d \tau \Delta \omega_{01}(\tau)\right]^{2}\right\rangle\right) \\
& =\exp \left(-\frac{2}{\hbar^{2}} S_{F_{z} F_{z}} t\right) .
\end{aligned}
$$

Note also that the noise correlator above is naturally symmetrized-the quantum asymmetry of the noise plays no role for this type of coupling. Equation (3.26) yields the dephasing rate

$$
\Gamma_{\varphi}=\left(2 / \hbar^{2}\right) S_{F_{z} F_{z}}=2 \theta_{0}^{2} S_{\dot{N}} .
$$

Using Eqs. (3.23) and (3.27), we find the interesting conclusion that the dephasing rate and measurement rates coincide,

$$
\Gamma_{\varphi} / \Gamma_{\text {meas }}=\left(4 / \hbar^{2}\right) S_{z z}^{I} S_{F_{z} F_{z}}=4 S_{\dot{N}} S_{\theta \theta}=1 .
$$

As we will see and prove rigorously, this represents the ideal quantum-limited case for QND qubit detection: the best one can do is measure as quickly as one dephases. In keeping with our earlier discussions, it represents the enforcement of the Heisenberg uncertainty 
principle. The faster you gain information about one variable, the faster you lose information about the conjugate variable. Note that, in general, the ratio $\Gamma_{\varphi} / \Gamma_{\text {meas }}$ will be larger than 1, as an arbitrary detector will not reach the quantum limit on its noise spectral densities. Such a nonideal detector produces excess back-action beyond what is required quantum mechanically.

In addition to the quantum noise point of view presented above, there is a second complementary way in which to understand the origin of measurement-induced dephasing (Stern et al., 1990), which is analogous to our description of loss of transverse spin coherence in the Stern-Gerlach experiment in Eq. (3.3). The measurement takes the incident wave, described by a coherent state $|\alpha\rangle$, to a reflected wave described by a (phase shifted) coherent state $\left|r_{\uparrow} \cdot \alpha\right\rangle$ or $\left|r_{\downarrow} \cdot \alpha\right\rangle$, where $r_{\uparrow / \downarrow}$ is the qubit-dependent reflection amplitude given in Eq. (3.12). Considering now the full state of the qubit-plus-detector, the measurement results in a state change:

$$
\begin{aligned}
\frac{1}{\sqrt{2}}(|\uparrow\rangle+|\downarrow\rangle) \otimes|\alpha\rangle \rightarrow & \frac{1}{\sqrt{2}}\left(e^{+i \omega_{01} t / 2}|\uparrow\rangle \otimes\left|r_{\uparrow} \cdot \alpha\right\rangle\right. \\
& \left.+e^{-i \omega_{01} t / 2}|\downarrow\rangle \otimes\left|r_{\downarrow} \cdot \alpha\right\rangle\right) .
\end{aligned}
$$

As $\left|r_{\uparrow} \cdot \alpha\right\rangle \neq\left|r_{\downarrow} \cdot \alpha\right\rangle$, the qubit has become entangled with the detector: the above state cannot be written as a product of a qubit state times a detector state. To assess the coherence of the final qubit state (i.e., the relative phase between $\uparrow$ and $\downarrow$ ), one looks at the off-diagonal matrix element of the qubit's reduced density matrix,

$$
\begin{aligned}
\rho_{\downarrow \uparrow} & \equiv \operatorname{Tr}_{\text {detector }}\langle\downarrow \mid \psi\rangle\langle\psi \mid \uparrow\rangle \\
& =\left(e^{+i \omega_{01} t} / 2\right)\left\langle r_{\uparrow} \cdot \alpha \mid r_{\downarrow} \cdot \alpha\right\rangle \\
& =\left(e^{+i \omega_{01} t} / 2\right) \exp \left[-|\alpha|^{2}\left(1-r_{\uparrow}^{*} r_{\downarrow}\right)\right] .
\end{aligned}
$$

In Eq. (3.31) we have used the usual expression for the overlap of two coherent states. We see that the measurement reduces the magnitude of $\rho_{\uparrow \downarrow}$ : this is dephasing. The amount of dephasing is directly related to the overlap between the different detector states that result when the qubit is up or down; this overlap can be straightforwardly found using Eq. (3.32) and $|\alpha|^{2}=\bar{N}$ $=\overline{\dot{N}} t$, where $\bar{N}$ is the mean number of photons that have reflected from the cavity after time $t$. We have

$$
\left|\exp \left[-|\alpha|^{2}\left(1-r_{\uparrow}^{*} r_{\downarrow}\right)\right]\right|=\exp \left[-2 \bar{N} \theta_{0}^{2}\right] \equiv \exp \left[-\Gamma_{\varphi} t\right],
$$

with the dephasing rate $\Gamma_{\varphi}$ given by

$$
\Gamma_{\varphi}=2 \theta_{0}^{2} \overline{\dot{N}}
$$

in complete agreement with the previous result in Eq. (3.27).

\section{Quantum limit relation for QND qubit state detection}

We now return to the ideal quantum limit relation of Eq. (3.28). As stated previously, this is a lower bound: quantum mechanics enforces the constraint that in a QND qubit measurement the best you can possibly do is measure as quickly as you dephase (Devoret and Schoelkopf, 2000; Korotkov and Averin, 2001; Makhlin et al., 2001; Averin, 2000b, 2003; Clerk et al., 2003),

$$
\Gamma_{\text {meas }} \leqslant \Gamma_{\varphi}
$$

While a detector with quantum-limited noise has an equality above, most detectors will be very far from this ideal limit, and will dephase the qubit faster than they acquire information about its state. We provide a proof of Eq. (3.35) in Sec. IV.B; for now, we note that its heuristic origin rests on the fact that both measurement and dephasing rely on the qubit becoming entangled with the detector. Consider again Eq. (3.29), describing the evolution of the qubit-detector system when the qubit is initially in a superposition of $\uparrow$ and $\downarrow$. To say that we have truly measured the qubit, the two detector states $\left|r_{\uparrow} \alpha\right\rangle$ and $\left|r_{\downarrow} \alpha\right\rangle$ need to correspond to different values of the detector output (i.e., phase shift $\theta$ in our example); this necessarily implies they are orthogonal. This in turn implies that the qubit is completely dephased: $\rho_{\uparrow \downarrow}=0$, just as we saw in Eq. (3.4) in the Stern-Gerlach example. Thus, measurement implies dephasing. The opposite is not true. The two states $\left|r_{\uparrow} \alpha\right\rangle$ and $\left|r_{\downarrow} \alpha\right\rangle$ could in principle be orthogonal without them corresponding to different values of the detector output (i.e., $\theta$ ). For example, the qubit may have become entangled with extraneous microscopic degrees of freedom in the detector. Thus, on a heuristic level, the origin of Eq. (3.35) is clear.

Returning to our one-sided cavity system, we see from Eq. (3.28) that the one-sided cavity detector reaches the quantum limit. It is natural to now ask why this is the case: Is there a general principle in action here that allows the one-sided cavity to reach the quantum limit? The answer is yes: reaching the quantum limit requires that there is no "wasted" information in the detector (Clerk et al., 2003). There should not exist any unmeasured quantity in the detector which could have been probed to learn more about the state of the qubit. In the single-sided cavity detector, information on the state of the qubit is only available in (that is, is entirely encoded in) the phase shift of the reflected beam; thus, there is no "wasted" information, and the detector does indeed reach the quantum limit.

To make this idea of "no wasted information" more concrete, we now consider a simple detector that fails to reach the quantum limit precisely due to wasted information. Consider again a $1 \mathrm{D}$ cavity system where now both mirrors are slightly transparent. Now, a wave incident at frequency $\omega_{R}$ on one end of the cavity will be partially reflected and partially transmitted. If the initial incident wave is described by a coherent state $|\alpha\rangle$, the scattered state can be described by a tensor product of the reflected wave's state and the transmitted wave's state, 


$$
|\alpha\rangle \rightarrow\left|r_{\sigma} \cdot \alpha\right\rangle\left|t_{\sigma} \cdot \alpha\right\rangle,
$$

where the qubit-dependent reflection and transmission amplitudes $r_{\sigma}$ and $t_{\sigma}$ are given by (Walls and Milburn, 1994)

$$
\begin{aligned}
& t_{\downarrow}=1 /\left(1+2 i A Q_{c}\right), \\
& r_{\downarrow}=2 i Q_{c} A /\left(1+2 i A Q_{c}\right),
\end{aligned}
$$

with $t_{\uparrow}=\left(t_{\downarrow}\right)^{*}$ and $r_{\uparrow}=\left(r_{\downarrow}\right)^{*}$. Note that the incident beam is almost perfectly transmitted: $\left|t_{\sigma}\right|^{2}=1-O\left(A Q_{c}\right)^{2}$.

Similar to the one-sided case, the two-sided cavity could be used to make a measurement by monitoring the phase of the transmitted wave. Using the expression for $t_{\sigma}$ above, we find that the qubit-dependent transmission phase shift is given by

$$
\tilde{\theta}_{\uparrow / \downarrow}= \pm \tilde{\theta}_{0}= \pm 2 A Q_{c},
$$

where again the two signs correspond to the two different qubit eigenstates. The phase shift for transmission is only half as large as in reflection so the Wigner delay time associated with transmission is

$$
\tilde{t}_{\mathrm{WD}}=2 / \kappa \text {. }
$$

Upon making the substitution of $\tilde{t}_{\mathrm{WD}}$ for $t_{\mathrm{WD}}$, the onesided cavity Eqs. (3.15) and (3.17) remain valid. However, the internal cavity photon-number shot noise remains fixed so that Eq. (3.18) becomes

$$
S_{n n}=2 \bar{n} \tilde{t}_{\mathrm{WD}}
$$

which means that

$$
S_{n n}=2 \overline{\dot{N}} \tilde{t}_{\mathrm{WD}}^{2}=2 S_{\dot{N}} \dot{t}_{\mathrm{WD}}^{2}
$$

and

$$
S_{F_{z} F_{z}}=2 \hbar^{2} A^{2} \omega_{c}^{2} \tilde{t}_{\mathrm{WD}}^{2} S_{\dot{N} \dot{N}}
$$

As a result the back-action dephasing doubles relative to the measurement rate and we have

$$
\Gamma_{\text {meas }} / \Gamma_{\varphi}=2 S_{N N} S_{\theta \theta}=\frac{1}{2} \text {. }
$$

Thus the two-sided cavity fails to reach the quantum limit by a factor of 2 .

Using the entanglement picture, we may again alternatively calculate the amount of dephasing from the overlap between the detector states corresponding to the qubit states $\uparrow$ and $\downarrow$ [cf. Eq. (3.31)]. We find

$$
\begin{aligned}
e^{-\Gamma_{\varphi^{t}}} & =\left|\left\langle t_{\uparrow} \alpha \mid t_{\downarrow} \alpha\right\rangle\left\langle r_{\uparrow} \alpha \mid r_{\downarrow} \alpha\right\rangle\right| \\
& =\exp \left[-|\alpha|^{2}\left(1-\left(t_{\uparrow}\right)^{*} t_{\downarrow}-\left(r_{\uparrow}\right)^{*} r_{\downarrow}\right)\right] .
\end{aligned}
$$

Note that both the changes in the transmission and reflection amplitudes contribute to the dephasing of the qubit. Using the above expressions, we find

$$
\Gamma_{\varphi} t=4\left(\tilde{\theta}_{0}\right)^{2}|\alpha|^{2}=4\left(\tilde{\theta}_{0}\right)^{2} \bar{N}=4\left(\tilde{\theta}_{0}\right)^{2} \overline{\dot{N}} t=2 \Gamma_{\text {meas }} t .
$$

Thus, in agreement with the quantum noise result, the two-sided cavity misses the quantum limit by a factor of 2.
Why does the two-sided cavity fail to reach the quantum limit? The answer is clear from Eq. (3.46): even though we are not monitoring it, there is information on the state of the qubit available in the phase of the reflected wave. Note from Eq. (3.38) that the magnitude of the reflected wave is weak $\left(\propto A^{2}\right)$, but (unlike the transmitted wave) the difference in the reflection phase associated with the two qubit states is large $( \pm \pi / 2)$. The "missing information" in the reflected beam makes a direct contribution to the dephasing rate [i.e., the second term in Eq. (3.46)], making it larger than the measurement rate associated with measurement of the transmission phase shift. In fact, there is an equal amount of information in the reflected beam as in the transmitted beam, so the dephasing rate is doubled. We thus have a concrete example of the general principle connecting a failure to reach the quantum limit to the presence of wasted information. Note that the application of this principle to generalized quantum point contact detectors is found in Clerk et al. (2003).

Returning to our cavity detector, we note in closing that it is often technically easier to work with the transmission of light through a two-sided cavity, rather than reflection from a one-sided cavity. One can still reach the quantum limit in the two-sided cavity case if one uses an asymmetric cavity in which the input mirror has much less transmission than the output mirror. Most photons are reflected at the input, but those that enter the cavity will almost certainly be transmitted. The price to be paid is that the input carrier power must be increased.

\section{Measurement of oscillator position using a resonant cavity}

The qubit measurement discussed previously was an example of a QND measurement: the back-action did not affect the observable being measured. We now consider the simplest example of a non-QND measurement, namely, the weak continuous measurement of the position of a harmonic oscillator. The detector will again be a parametrically coupled resonant cavity, where the position of the oscillator $x$ changes the frequency of the cavity as per Eq. (3.11) [see, e.g., Tittonen et al. (1999)]. Similarly to the qubit case, for a sufficiently weak coupling the phase shift of the reflected beam from the cavity will depend linearly on the position $x$ of the oscillator [cf. Eq. (3.13)]; by reading out this phase, we may thus measure $x$. The origin of back-action noise is the same as before, namely, photon shot noise in the cavity. Now, however, this represents a random force which changes the momentum of the oscillator. During the subsequent time evolution these random force perturbations will reappear as random fluctuations in the position. Thus the measurement is not QND. This will mean that the minimum uncertainty of even an ideal measurement is larger (by exactly a factor of 2) than the "true" quantum uncertainty of the position (i.e., the ground-state uncertainty). This is known as the standard quantum limit on weak continuous position detection. It is also an example of a general principle that a linear phase- 
preserving amplifier necessarily adds noise, and that the minimum added noise exactly doubles the output noise for the case where the input is vacuum (i.e., zero-point) noise. A more general discussion of the quantum limit on amplifiers and position detectors will be presented in Sec. V.

We start by emphasizing that we are speaking here of a weak continuous measurement of the oscillator position. The measurement is sufficiently weak that the position undergoes many cycles of oscillation before significant information is acquired. Thus we are not talking about the instantaneous position but rather the overall amplitude and phase, or more precisely the two quadrature amplitudes describing the smooth envelope of the motion,

$$
\hat{x}(t)=\hat{X}(t) \cos (\Omega t)+\hat{Y}(t) \sin (\Omega t) .
$$

One can easily show that, for an oscillator, the two quadrature amplitudes $\hat{X}$ and $\hat{Y}$ are canonically conjugate and hence do not commute with each other,

$$
[\hat{X}, \hat{Y}]=i \hbar / M \Omega=2 i x_{\mathrm{ZPF}}^{2} \text {. }
$$

As the measurement is both weak and continuous, it will yield information on both $\hat{X}$ and $\hat{Y}$. As such, one is effectively trying to simultaneously measure two incompatible observables. This basic fact is intimately related to the property mentioned above, that even a completely ideal weak continuous position measurement will have a total uncertainty that is twice the zero-point uncertainty.

We are now ready to start our heuristic analysis of position detection using a cavity detector; relevant calculational details presented in Appendix E.3. Consider first the mechanical oscillator we wish to measure. We take it to be a simple harmonic oscillator of natural frequency $\Omega$ and mechanical damping rate $\gamma_{0}$. For weak damping, and at zero coupling to the detector, the spectral density of the oscillator's position fluctuations is given by Eq. (2.4) with the delta function replaced by a Lorentzian

$$
\begin{aligned}
S_{x x}[\omega]= & x_{\mathrm{ZPF}}^{2}\left\{n_{B}(\hbar \Omega) \frac{\gamma_{0}}{(\omega+\Omega)^{2}+\left(\gamma_{0} / 2\right)^{2}}\right. \\
& \left.+\left[n_{B}(\hbar \Omega)+1\right] \frac{\gamma_{0}}{(\omega-\Omega)^{2}+\left(\gamma_{0} / 2\right)^{2}}\right\} .
\end{aligned}
$$

When we now weakly couple the oscillator to the cavity [as per Eq. (3.11), with $\left.\hat{z}=\hat{x} / x_{\mathrm{ZPF}}\right]$ and drive the cavity on resonance, the phase shift $\theta$ of the reflected beam will be proportional to $x$ (i.e., $\delta \theta(t)=[d \theta / d x] x(t)$ ). As such, the oscillator's position fluctuations will cause ad-

\footnotetext{
${ }^{5}$ This form is valid only for weak damping because we are assuming that the oscillator frequency is still sharply defined. We have evaluated the Bose-Einstein factor exactly at frequency $\Omega$ and have assumed that the Lorentzian centered at positive (negative) frequency has negligible weight at negative (positive) frequencies.
}

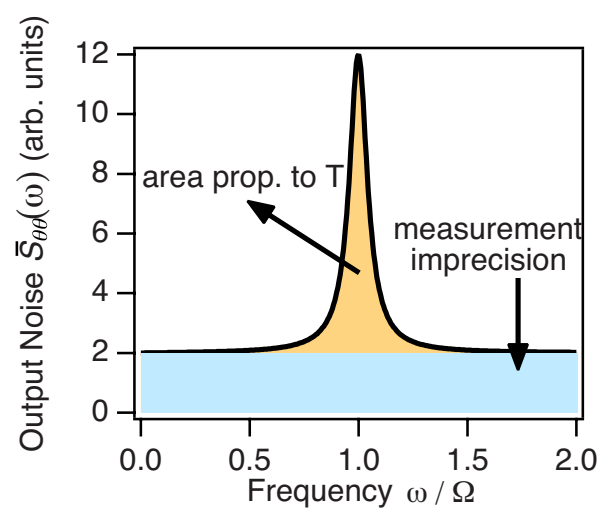

FIG. 4. (Color online) Spectral density of the symmetrized output noise $\bar{S}_{\theta \theta}[\omega]$ of a linear position detector. The oscillator's noise appears as a Lorentzian on top of a noise floor (i.e., the measurement imprecision). As discussed in the text, the width of the peak is proportional to the oscillator damping rate $\gamma_{0}$, while the area under the peak is proportional to temperature. This latter fact can be used to calibrate the response of the detector.

ditional fluctuations of the phase $\theta$, over and above the intrinsic shot noise-induced phase fluctuations $S_{\theta \theta}$. We consider the usual case where the noise spectrometer being used to measure the noise in $\theta$ (i.e., the noise in the homodyne current) measures the symmetric-infrequency noise spectral density; as such, it is the symmetric-in-frequency position noise that we detect. In the classical limit $k_{B} T \gg \hbar \Omega$, this is given by

$$
\begin{aligned}
\bar{S}_{x x}[\omega] & \equiv \frac{1}{2}\left(S_{x x}[\omega]+S_{x x}[-\omega]\right) \\
& \approx \frac{k_{B} T}{2 M \Omega^{2}} \frac{\gamma_{0}}{(|\omega|-\Omega)^{2}+\left(\gamma_{0} / 2\right)^{2}} .
\end{aligned}
$$

If we ignore back-action effects, we expect to see this Lorentzian profile riding on top of the background imprecision noise floor; this is shown in Fig. 4.

Note that additional stages of amplification would also add noise, and would thus further augment this background noise floor. If we subtract off this noise floor, the full width at half maximum of the curve will give the damping parameter $\gamma_{0}$, and the area under the experimental curve,

$$
\int_{-\infty}^{\infty} \frac{d \omega}{2 \pi} \bar{S}_{x x}[\omega]=\frac{k_{B} T}{M \Omega^{2}}
$$

measures the temperature. What the experimentalist actually plots in making such a curve is the output of the entire detector-plus-following-amplifier chain. Importantly, if the temperature is known, then the area of the measured curve can be used to calibrate the coupling of the detector and the gain of the total overall amplifier chain (see, e.g., LaHaye et al., 2004; Flowers-Jacobs et al. 2007). One can thus make a calibrated plot where the measured output noise is referred back to the oscillator position. 
Consider now the case where the oscillator is at zero temperature. Equation (3.50) then yields for the symmetrized noise spectral density

$$
\bar{S}_{x x}^{0}[\omega]=x_{\mathrm{ZPF}}^{2} \frac{\gamma_{0} / 2}{(|\omega|-\Omega)^{2}+\left(\gamma_{0} / 2\right)^{2}} .
$$

One might expect that one could see this Lorentzian directly in the output noise of the detector (i.e., the $\theta$ noise), above the measurement-imprecision noise floor. However, this neglects the effects of measurement backaction. From the classical equation of motion we expect the response of the oscillator to the back-action force $F=F_{z} / x_{\mathrm{ZPF}}$ [cf. Eq. (3.16)] at frequency $\omega$ to produce an additional displacement $\delta x[\omega]=\chi_{x x}[\omega] F[\omega]$, where $\chi_{x x}[\omega]$ is the mechanical susceptibility

$$
\chi_{x x}[\omega] \equiv \frac{1}{M} \frac{1}{\Omega^{2}-\omega^{2}-i \gamma_{0} \omega} .
$$

These extra oscillator fluctuations will show up as additional fluctuations in the output of the detector. For simplicity, we focus on this noise at the oscillator's resonance frequency $\Omega$. As a result of the detector's backaction, the total measured position noise (i.e., inferred spectral density) at the frequency $\Omega$ is given by

$$
\begin{aligned}
\bar{S}_{x x, \text { tot }}[\Omega]= & \bar{S}_{x x}^{0}[\Omega]+\frac{\left|\chi_{x x}[\Omega]\right|^{2}}{2}\left[S_{F F}[+\Omega]+S_{F F}[-\Omega]\right] \\
& +\frac{1}{2}\left[S_{x x}^{\mathrm{I}}[+\Omega]+S_{x x}^{\mathrm{I}}[-\Omega]\right] \\
= & \bar{S}_{x x}^{0}[\Omega]+\bar{S}_{x x, \text { add }}[\Omega] .
\end{aligned}
$$

The first term here is just the intrinsic zero-point noise of the oscillator,

$$
\bar{S}_{x x}^{0}[\Omega]=2 x_{\mathrm{ZPF}}^{2} / \gamma_{0}=\hbar \chi_{x x}[\Omega] \mid .
$$

The second term $\bar{S}_{x x, \text { add }}$ is the total noise added by the measurement, and includes both the measurement imprecision $S_{x x}^{\mathrm{I}} \equiv S_{z z}^{\mathrm{I}} x_{\mathrm{ZPF}}^{2}$ and the extra fluctuations caused by the back-action. We stress that $\bar{S}_{x x, \text { tot }}$ corresponds to a position noise spectral density inferred from the output of the detector: one simply scales the spectral density of total output fluctuations $\bar{S}_{\theta \theta \text {,tot }}[\Omega]$ by $(d \theta / d x)^{2}$.

Implicit in Eq. (3.57) is the assumption that the backaction noise and the imprecision noise are uncorrelated and thus add in quadrature. It is not obvious that this is correct, since in the cavity detector the back-action noise and output shot noise are both caused by the vacuum noise in the beam incident on the cavity. It turns out that there are indeed correlations, however, the symmetrized (i.e., classical) correlator $\bar{S}_{\theta F}$ does vanish for our choice of a resonant cavity drive. Further, Eq. (3.55) assumes that the measurement does not change the damping rate of the oscillator. Again, while this will not be true for an arbitrary detector, it is the case here for the cavity detector when (as we have assumed) it is driven on resonance. Details justifying both these statements are given

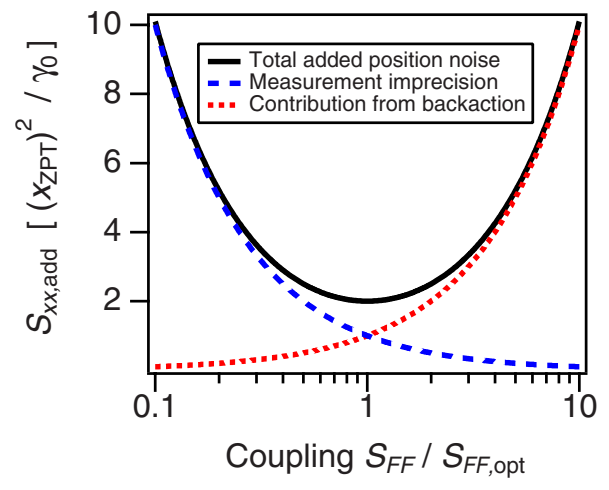

FIG. 5. (Color online) Noise power of the added position noise of a linear position detector, evaluated at the oscillator's resonance frequency $\left(\bar{S}_{x x \text {,add }}[\Omega]\right)$ as a function of the magnitude of the back-action noise spectral density $S_{F F} . S_{F F}$ is proportional to the oscillator-detector coupling, and in the case of the cavity detector is also proportional to the power incident on the cavity. The optimal value of $S_{F F}$ is given by $S_{F F, \text { opt }}=\hbar M \Omega \gamma_{0} / 2$ [cf. Eq. (3.59)]. We have assumed that there are no correlations between measurement-imprecision noise and back-action noise, as is appropriate for the cavity detector.

in Appendix E; the more general case with nonzero noise correlations and back-action damping is discussed in Sec. V.E.

Assuming we have a quantum-limited detector that obeys Eq. (3.9) (i.e., $S_{x x}^{\mathrm{I}} S_{F F}=\hbar^{2} / 4$ ) and that the shot noise is symmetric in frequency, the added position noise spectral density at resonance [i.e., second term in Eq. (3.56)] becomes

$$
\bar{S}_{x x, \text { add }}[\Omega]=\left[|\chi[\Omega]|^{2} S_{F F}+\frac{\hbar^{2}}{4} \frac{1}{S_{F F}}\right] .
$$

Recall from Eq. (3.19) that the back-action noise is proportional to the coupling of the oscillator to the detector and to the intensity of the drive on the cavity. The added position-uncertainty noise is plotted in Fig. 5 as a function of $S_{F F}$. We see that for high drive intensity the backaction noise dominates the position uncertainty, while for low drive intensity the output shot noise (the last term in the equation above) dominates.

The added noise (and hence the total noise $\bar{S}_{x x \text {,tot }}[\Omega]$ ) is minimized when the drive intensity is tuned so that $S_{F F}$ is equal to $S_{F F, \text { opt }}$, with

$$
S_{F F, \text { opt }}=\hbar / 2 \mid \chi_{x x}[\Omega]=(\hbar / 2) M \Omega \gamma_{0} .
$$

The more heavily damped is the oscillator, the less susceptible it is to back-action noise and hence the higher is the optimal coupling. At the optimal coupling strength, the measurement-imprecision noise and back-action noise each make equal contributions to the added noise, yielding

$$
\bar{S}_{x x, \text { add }}[\Omega]=\hbar / M \Omega \gamma_{0}=\bar{S}_{x x}^{0}[\Omega] .
$$

Thus, the spectral density of the added position noise is exactly equal to the noise power associated with the oscillator's zero-point fluctuations. This represents a mini- 
mum value for the added noise of any linear position detector, and is referred to as the standard quantum limit on position detection. Note that this limit only involves the added noise of the detector, and thus has nothing to do with the initial temperature of the oscillator.

We emphasize that to reach the above quantum limit on weak continuous position detection one needs the detector itself to be quantum limited, i.e., the product $S_{F F} S_{x x}^{\mathrm{I}}$ must be as small as is allowed by quantum mechanics, namely, $\hbar^{2} / 4$. Having a quantum-limited detector, however, is not enough: in addition, one must be able to achieve sufficiently strong coupling to reach the optimum given in Eq. (3.59). Further, the measured output noise must be dominated by the output noise of the cavity, not by the added noise of following amplifier stages.

A related, stronger quantum limit refers to the total inferred position noise from the measurement $\bar{S}_{x x, \text { tot }}[\omega]$. It follows from Eqs. (3.60) and (3.56) that at resonance the smallest this can be is twice the oscillator's zeropoint noise

$$
\bar{S}_{x x, \text { tot }}[\Omega]=2 \bar{S}_{x x}^{0}[\Omega] .
$$

Half the noise here is from the oscillator itself, half is from the added noise of the detector. It is even more challenging to reach this quantum limit: one needs to both reach the quantum limit on the added noise and cool the oscillator to its ground state.

Finally, we emphasize that the optimal value of the coupling derived above was specific to the choice of minimizing the total position noise power at the resonance frequency. If a different frequency had been chosen, the optimal coupling would have been different; one again finds that the minimum possible added noise corresponds to the ground-state noise at that frequency. It is interesting to ask what the total position noise would be as a function of frequency, assuming that the coupling has been optimized to minimize the noise at the resonance frequency, and that the oscillator is initially in the ground state. From our results above we have

$$
\begin{aligned}
\bar{S}_{x x, \text { tot }}[\omega]= & x_{\mathrm{ZPF}}^{2} \frac{\gamma_{0} / 2}{(|\omega|-\Omega)^{2}+\left(\gamma_{0} / 2\right)^{2}} \\
& +\frac{\hbar}{2}\left[\frac{\left|\chi_{x x}[\omega]\right|^{2}}{\left|\chi_{x x}[\Omega]\right|}+\left|\chi_{x x}[\Omega]\right|\right] \\
\approx & \frac{x_{\mathrm{ZPF}}^{2}}{\gamma_{0}}\left\{1+3 \frac{\left(\gamma_{0} / 2\right)^{2}}{(|\omega|-\Omega)^{2}+\left(\gamma_{0} / 2\right)^{2}}\right\},
\end{aligned}
$$

which is plotted in Fig. 6. Assuming that the detector is quantum limited, one sees that the Lorentzian peak rises above the constant background by a factor of 3 when the coupling is optimized to minimize the total noise power at resonance. This represents the best one can do when continuously monitoring zero-point position fluctuations. Note that the value of this peak-to-floor ratio is a direct consequence of two simple facts which hold for an

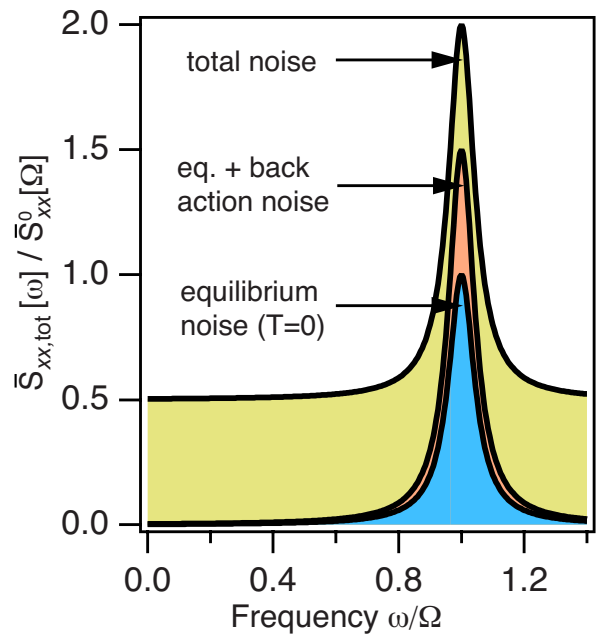

FIG. 6. (Color online) Spectral density of measured position fluctuations of a harmonic oscillator $\bar{S}_{x x \text {,tot }}[\omega]$ as a function of frequency $\omega$, for a detector which reaches the quantum limit at the oscillator frequency $\Omega$. We have assumed that without the coupling to the detector the oscillator would be in its ground state. The $y$ axis has been normalized by the zero-point position noise spectral density $\bar{S}_{x x}^{0}[\omega]$, evaluated at $\omega=\Omega$. One clearly sees that the total noise at $\Omega$ is twice the zero-point value, and that the peak of the Lorentzian rises a factor of 3 above the background. This background represents the measurement imprecision and is equal to $1 / 2$ of $\bar{S}_{x x}^{0}(\Omega)$.

optimal coupling at the quantum limit: (i) the total added noise at resonance (back-action plus measurement imprecision) is equal to the zero-point noise and (ii) back-action and measurement imprecision make equal contributions to the total added noise. Somewhat surprisingly, the same maximum peak-to-floor ratio is obtained when one tries to continuously monitor coherent qubit oscillations with a linear detector which is transversely coupled to the qubit (Korotkov and Averin, 2001); this is also a non-QND situation. Finally, if one only wants to detect the noise peak (as opposed to making a continuous quantum-limited measurement), one could use two independent detectors coupled to the oscillator and look at the cross correlation between the two output noises: in this case, there need not be any noise floor (Jordan and Büttiker, 2005a; Doiron et al., 2007).

In Table III, we give a summary of recent experiments which approach the quantum limit on weak continuous position detection of a mechanical resonator. Note that in many of these experiments the effects of detector back-action were not seen. This could either be the result of too low a detector-oscillator coupling or due to the presence of excessive thermal noise. As shown, the back-action force noise serves to slightly heat the oscillator. If it is already at an elevated temperature due to thermal noise, this additional heating can be hard to resolve.

In closing, we stress that this section has given only a rudimentary introduction to the quantum limit on position detection. A complete discussion which treats the 
TABLE III. Synopsis of recent experiments approaching the quantum limit on continuous position detection of a mechanical resonator. The second column corresponds to the best measurement-imprecision noise spectral density $\bar{S}_{x x}^{I}$ achieved in the experiment. This value is compared against the zero-point position noise spectral density $\bar{S}_{x x}^{0}$, calculated using the total measured resonator damping (which may include a back-action contribution). All spectral densities are at the oscillator's resonance frequency $\Omega$. As discussed in the text, there is no quantum limit on how small one can make $\bar{S}_{x x}^{I}$; for an ideal detector, one needs to tune the detector-resonator coupling so that $\bar{S}_{x x}^{I}=\bar{S}_{x x}^{0} / 2$ in order to reach the quantum limit on position detection. The third column presents the product of the measured imprecision noise (unless otherwise noted) and measured back-action noises, divided by $\hbar / 2$; this quantity must be one to achieve the quantum limit on the added noise.

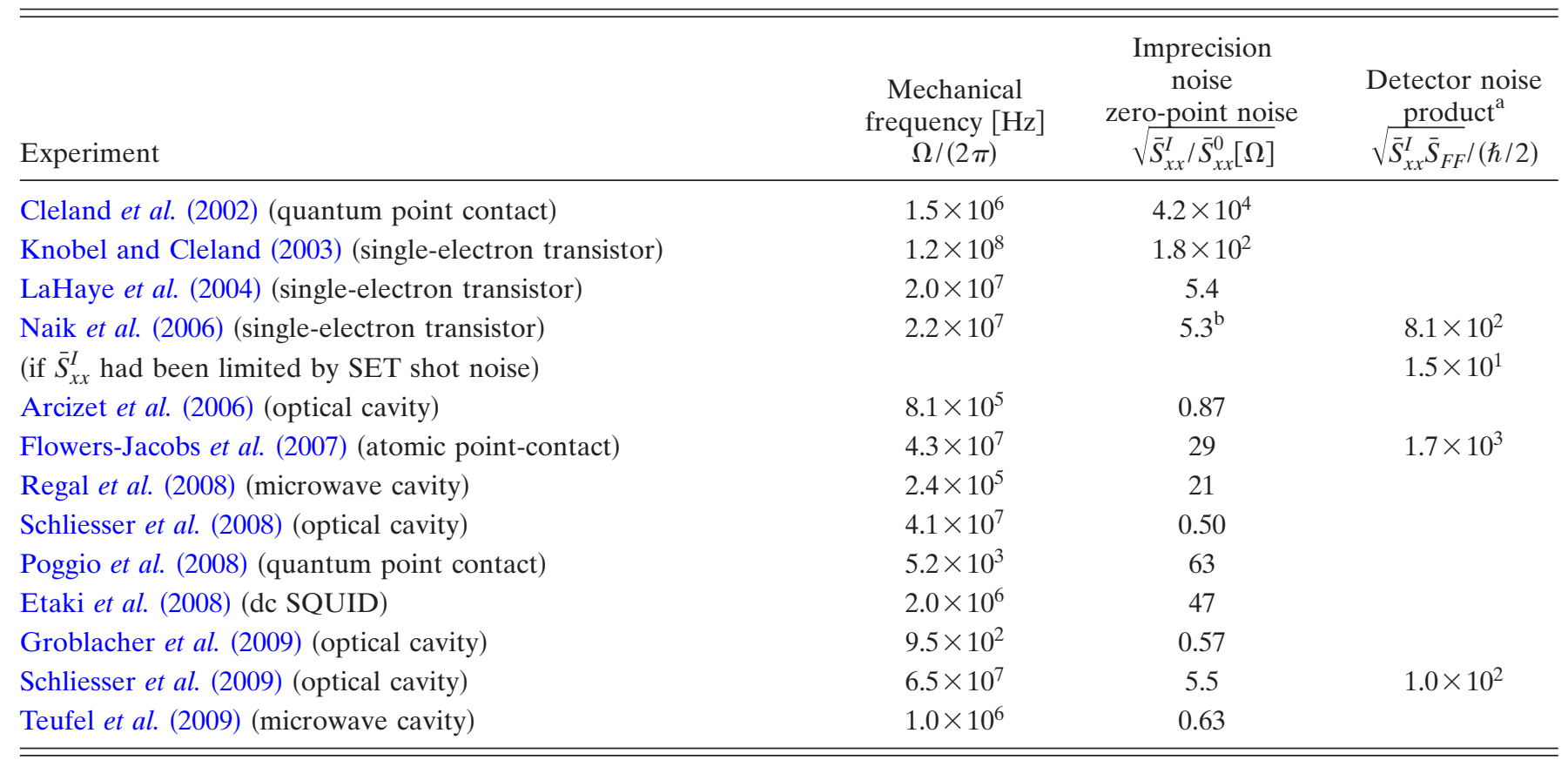

${ }^{\mathrm{a}} \mathrm{A}$ blank value in this column indicates that back-action was not measured in the experiment.

${ }^{\mathrm{b}}$ Note that back-action effects dominated the mechanical $Q$ in this measurement, lowering it from $1.2 \times 10^{5}$ to $\sim 4.2 \times 10^{2}$. If one compares the imprecision against the zero-point noise of the uncoupled mechanical resonator, one finds $\sqrt{\bar{S}_{x x}^{I} / \bar{S}_{x x}^{0}[\Omega]} \sim 0.33$.

important topics of back-action damping, effective temperature, noise cross correlation, and power gain is given in Sec. V.E.

\section{GENERAL LINEAR-RESPONSE THEORY}

\section{A. Quantum constraints on noise}

In this section, we further develop the connection between quantum limits and noise discussed previously, focusing now on a more general approach. As before, we emphasize the idea that reaching the quantum limit requires a detector having quantum-ideal noise properties. The approach here is different from typical treatments in the quantum optics literature (Gardiner and Zoller, 2000; Haus, 2000), and uses nothing more than features of quantum linear response. Our discussion here will expand upon Clerk et al. (2003) and Clerk (2004); somewhat similar approaches to quantum measurement are also discussed by Braginsky and Khalili (1992) and Averin (2003).

In this section, we start by heuristically sketching how constraints on noise [similar to Eq. (3.9) for the cavity detector] can emerge directly from the Heisenberg uncertainty principle. We then present a rigorous and gen- eral quantum constraint on noise. We introduce both the notion of a generic linear-response detector and the basic quantum constraint on detector noise. Next, we discuss how this noise constraint leads to the quantum limit on QND state detection of a qubit. The quantum limit on a linear amplifier (or a position detector) is discussed in the next section.

\section{Heuristic weak-measurement noise constraints}

As stressed in the introduction, there is no fundamental quantum limit on the accuracy with which a given observable can be measured, at least not within the framework of nonrelativistic quantum mechanics. For example, one can, in principle, measure the position of a particle to arbitrary accuracy in the course of a projection measurement. However, the situation is different when we specialize to continuous non-QND measurements. Such a measurement can be envisaged as a series of instantaneous measurements, in the limit where the spacing between the measurements $\delta t$ is taken to zero. Each measurement in the series has a limited resolution and perturbs the conjugate variables, thereby affecting the subsequent dynamics and measurement results. We 


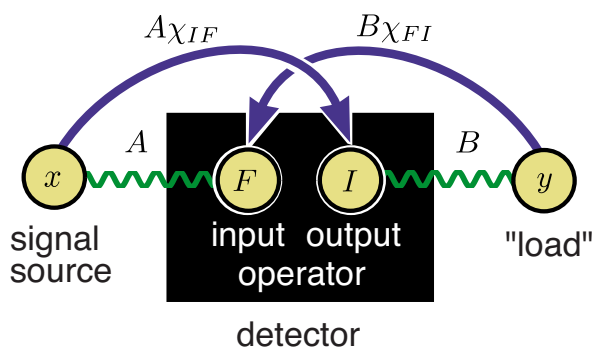

FIG. 7. (Color online) Schematic of a generic linear-response detector.

discuss this for the example of a series of position measurements of a free particle.

After initially measuring the position with an accuracy $\Delta x$, the momentum suffers a random perturbation of size $\Delta p \geqslant \hbar /(2 \Delta x)$. Consequently, a second position measurement taking place a time $\delta t$ later will have an additional uncertainty of size $\delta t(\Delta p / m) \sim \hbar \delta t / m \Delta x$. Thus, when one is trying to obtain a good estimate of the position by averaging several such measurements, it is not optimal to make $\Delta x$ too small, because otherwise this additional perturbation, called the back-action of the measurement device, will become large. The back-action can be described as a random force $\Delta F=\Delta p / \delta t$. A meaningful limit $\delta t \rightarrow 0$ is obtained by keeping both $\Delta x^{2} \delta t$ $\equiv \bar{S}_{x x}$ and $\Delta p^{2} / \delta t \equiv \bar{S}_{F F}$ fixed. In this limit, the deviations $\delta x(t)$ describing the finite measurement accuracy and the fluctuations of the back-action force $F$ can be described as white noise processes, $\langle\delta x(t) \delta x(0)\rangle=\bar{S}_{x x} \delta(t)$ and $\langle F(t) F(0)\rangle=\bar{S}_{F F} \delta(t)$. The Heisenberg uncertainty relation $\Delta p \Delta x \geqslant \hbar / 2$ then implies $\bar{S}_{x x} \bar{S}_{F F} \geqslant \hbar^{2} / 4$ (Braginsky and Khalili, 1992). Note that this is completely analogous to the relation (3.9) we derived for the resonant cavity detector using the fundamental number-phase uncertainty relation. In this section, we derive rigorously more general quantum limit relations on noise spectral densities of this form.

\section{Generic linear-response detector}

To rigorously discuss the quantum limit, we start with a description of a detector that is as general as possible. To that end, we think of a detector as some physical system (described by some unspecified Hamiltonian $\hat{H}_{\text {det }}$ and some unspecified density matrix $\hat{\rho}_{0}$ ) which is time independent in the absence of coupling to the signal source. The detector has both an input port, characterized by an operator $\hat{F}$, and an output port, characterized by an operator $\hat{I}$ (see Fig. 7). The output operator $\hat{I}$ is simply the quantity that is read out at the output of the detector (e.g., the current in a single-electron transistor, or the phase shift in the cavity detector of the previous section). The input operator $\hat{F}$ is the detector quantity that directly couples to the input signal (e.g., the qubit) and that causes a back-action disturbance of the signal source; in the cavity example of the previous section, we had $\hat{F}=\hat{n}$, the cavity photon number. As we are interested in weak couplings, we assume a simple bilinear form for the detector-signal interaction Hamiltonian

$$
\hat{H}_{\text {int }}=A \hat{x} \hat{F} \text {. }
$$

Here the operator $\hat{x}$ (which is not necessarily a position operator) carries the input signal. Note that because $\hat{x}$ belongs to the signal source, it necessarily commutes with the detector variables $\hat{I}, \hat{F}$.

We always assume the coupling strength $A$ to be small enough that we can accurately describe the output of the detector using linear response. ${ }^{6}$ We thus have

$$
\langle\hat{I}(t)\rangle=\langle\hat{I}\rangle_{0}+A \int d t^{\prime} \chi_{I F}\left(t-t^{\prime}\right)\left\langle\hat{x}\left(t^{\prime}\right)\right\rangle,
$$

where $\langle\hat{I}\rangle_{0}$ is the input-independent value of the detector output at zero coupling and $\chi_{I F}(t)$ is the linear-response susceptibility or gain of our detector. Note that Clerk et al. (2003) and Clerk (2004) denoted this gain coefficient $\lambda$. Using standard time-dependent perturbation theory in the coupling $\hat{H}_{\text {int }}$, one can easily derive Eq. (4.2), with $\chi_{I F}(t)$ given by a Kubo-like formula,

$$
\chi_{I F}(t)=-(i / \hbar) \theta(t)\langle[\hat{I}(t), \hat{F}(0)]\rangle_{0} .
$$

Here and in what follows the operators $\hat{I}$ and $\hat{F}$ are Heisenberg operators with respect to the detector Hamiltonian, and the subscript 0 indicates an expectation value with respect to the density matrix of the uncoupled detector.

As discussed, there will be unavoidable noise in both the input and output ports of our detector. This noise is subject to quantum-mechanical constraints, and its presence is what limits our ability to make a measurement or amplify a signal. We thus need to quantitatively characterize the noise in both these ports. Recall from the discussion in Sec. II.B that it is the symmetric-in-frequency part of a quantum noise spectral density which plays a role akin to classical noise. We thus want to characterize the symmetrized noise correlators of our detector (denoted as always with an overbar). Redefining these operators so that their average value is zero at zero coupling (i.e., $\hat{F} \rightarrow \hat{F}-\langle\hat{F}\rangle_{0}, \hat{I} \rightarrow \hat{I}-\langle\hat{I}\rangle_{0}$ ), we have

$$
\begin{aligned}
& \bar{S}_{F F}[\omega] \equiv \frac{1}{2} \int_{-\infty}^{\infty} d t e^{i \omega t}\langle\{\hat{F}(t), \hat{F}(0)\}\rangle_{0}, \\
& \bar{S}_{I I}[\omega] \equiv \frac{1}{2} \int_{-\infty}^{\infty} d t e^{i \omega t}\langle\{\hat{I}(t), \hat{I}(0)\}\rangle_{0},
\end{aligned}
$$

\footnotetext{
${ }^{6}$ The precise condition for the breakdown of linear response will depend on specific details of the detector. For example, in the cavity detector discussed in Sec. III.B, one would need the dimensionless coupling $A$ to satisfy $A \ll 1 / Q_{c}\langle\hat{z}\rangle$ to ensure that the nonlinear dependence of the phase shift $\theta$ on the signal $\langle\hat{z}\rangle$ is negligible. This translates to the signal modulating the cavity frequency by an amount much smaller than its linewidth $\kappa$.
} 


$$
\bar{S}_{I F}[\omega] \equiv \frac{1}{2} \int_{-\infty}^{\infty} d t e^{i \omega t}\langle\{\hat{I}(t), \hat{F}(0)\}\rangle_{0},
$$

where $\{$,$\} indicates the anticommutator, \bar{S}_{I I}$ represents the intrinsic noise in the output of the detector, and $\bar{S}_{F F}$ describes the back-action noise seen by the source of the input signal. In general, there will be some correlation between these two kinds of noise; this is described by the cross correlator $\bar{S}_{I F}$.

Finally, we must also allow for the possibility that our detector could operate in reverse (i.e., with input and output ports playing opposite roles). We thus introduce the reverse gain $\chi_{F I}$ of our detector. This is the response coefficient describing an experiment where we couple our input signal to the output port of the detector (i.e., $\left.\hat{H}_{\text {int }}=A \hat{x} \hat{I}\right)$, and attempt to observe something at the input port [i.e., in $\langle\hat{F}(t)\rangle]$. In complete analogy to Eq. (4.2), one would then have

$$
\langle\hat{F}(t)\rangle=\langle\hat{F}\rangle_{0}+A \int d t^{\prime} \chi_{F I}\left(t-t^{\prime}\right)\left\langle\hat{x}\left(t^{\prime}\right)\right\rangle
$$

with

$$
\chi_{F I}(t)=-(i / \hbar) \theta(t)\langle[\hat{F}(t), \hat{I}(0)]\rangle_{0} .
$$

Note that if our detector is in a time-reversal symmetric, thermal equilibrium state, then Onsager reciprocity relations would imply either $\chi_{I F}=\chi_{F I}^{*}$ (if $I$ and $F$ have the same parity under time reversal) or $\chi_{I F}=-\chi_{F I}^{*}$ (if $I$ and $F$ have the opposite parity under time reversal) [see, e.g., Pathria (1996)]. Thus, if the detector is in equilibrium, the presence of gain necessarily implies the presence of reverse gain. Nonzero reverse gain is also found in many standard classical electrical amplifiers such as op-amps (Boylestad and Nashelsky, 2006).

The reverse gain is something that we must worry about even if we are not interested in operating our detector in reverse. To see why, note that to make a measurement of the output operator $\hat{I}$, we must necessarily couple to it in some manner. If $\chi_{F I} \neq 0$, the noise associated with this coupling could in turn lead to additional back-action noise in the operator $\hat{F}$. Even if the reverse gain did nothing but amplify vacuum noise entering the output port, this would heat up the system being measured at the input port and hence produce excess backaction. Thus, the ideal situation is to have $\chi_{F I}=0$, implying a high asymmetry between the input and output of the detector, and requiring the detector to be in a state far from thermodynamic equilibrium. We note that almost all mesoscopic detectors that have been studied in detail (e.g., single-electron transistors and generalized quantum point contacts) have been found to have a vanishing reverse gain: $\chi_{F I}=0$ (Clerk et al., 2003). For this reason, we often focus on the ideal (but experimentally relevant) situation where $\chi_{F I}=0$ in what follows.

Before proceeding, it is worth emphasizing that there is a relation between the detector gains $\chi_{I F}$ and $\chi_{F I}$ and the unsymmetrized $I-F$ quantum noise correlator, $S_{I F}[\omega]$. This spectral density, which need not be symmetric in frequency, is defined as

$$
S_{I F}[\omega]=\int_{-\infty}^{\infty} d t e^{i \omega t}\langle\hat{I}(t) \hat{F}(0)\rangle_{0} .
$$

Using the definitions, one can easily show that

$$
\begin{aligned}
& \bar{S}_{I F}[\omega]=\frac{1}{2}\left[S_{I F}[\omega]+S_{I F}[-\omega]^{*}\right], \\
& \chi_{I F}[\omega]-\chi_{F I}[\omega]^{*}=-(i / \hbar)\left[S_{I F}[\omega]-S_{I F}[-\omega]^{*}\right] .
\end{aligned}
$$

Thus, while $\bar{S}_{I F}$ represents the classical part of the $I-F$ quantum noise spectral density, the gains $\chi_{I F}, \chi_{F I}$ are determined by the quantum part of this spectral density. This also demonstrates that though the gains have an explicit factor of $1 / \hbar$ in their definitions, they have a well-defined $\hbar \rightarrow 0$ limit, as the asymmetric-in-frequency part of $S_{I F}[\omega]$ vanishes in this limit.

\section{Quantum constraint on noise}

Despite having said nothing about the detector's Hamiltonian or state (except that it is time independent), we can nonetheless derive a general quantum constraint on its noise properties. Note first that for purely classical noise spectral densities one always has the inequality

$$
\bar{S}_{I I}[\omega] \bar{S}_{F F}[\omega]-\left|\bar{S}_{I F}[\omega]\right|^{2} \geqslant 0 .
$$

This simply expresses the fact that the correlation between two different noisy quantities cannot be arbitrarily large; it follows immediately from the Schwartz inequality. In the quantum case, this simple constraint becomes modified whenever there is an asymmetry between the detector's gain and reverse gain. This asymmetry is parametrized by the quantity $\tilde{\chi}_{I F}[\omega]$,

$$
\tilde{\chi}_{I F}[\omega] \equiv \chi_{I F}[\omega]-\left(\chi_{F I}[\omega]\right)^{*} .
$$

We show below that the following quantum noise inequality (involving symmetrized noise correlators) is always valid [see also Eq. (6.36) in Braginsky and Khalili (1996)],

$$
\begin{aligned}
& \bar{S}_{I I}[\omega] \bar{S}_{F \digamma}[\omega]-\left|\bar{S}_{I F}[\omega]\right|^{2} \\
& \geqslant\left|\frac{\hbar \tilde{\chi}_{I F}[\omega]}{2}\right|^{2}\left(1+\Delta\left[\frac{\bar{S}_{I \digamma}[\omega]}{\hbar \tilde{\chi}_{I F}[\omega] / 2}\right]\right),
\end{aligned}
$$

where

$$
\Delta[z]=\left[\left|1+z^{2}\right|-\left(1+|z|^{2}\right)\right] / 2 .
$$

To interpret the quantum noise inequality Eq. (4.11), note that $1+\Delta[z] \geqslant 0$. Equation (4.11) thus implies that if our detector has gain and does not have a perfect symmetry between input and output (i.e., $\chi_{I F} \neq \chi_{F I}^{*}$ ), then it must in general have a minimum amount of back-action and output noise; moreover, these two noises cannot be perfectly anticorrelated. As shown in the following sections, this constraint on the noise of a detector directly 
leads to quantum limits on various different measurement tasks. Note that in the zero-frequency limit $\tilde{\chi}_{I F}$ and $\bar{S}_{I F}$ are both real, implying that the term involving $\Delta$ in Eq. (4.11) vanishes. The result is a simpler-looking inequality found elsewhere (Averin, 2003; Clerk et al., 2003; Clerk, 2004).

While Eq. (4.11) may appear reminiscent of the standard fluctuation-dissipation theorem, its origin is quite different: in particular, the quantum noise constraint applies irrespective of whether the detector is in equilibrium. Equation (4.11) instead follows directly from Heisenberg's uncertainty relation applied to the frequency representation of the operators $\hat{I}$ and $\hat{F}$. In its most general form, the Heisenberg uncertainty relation gives a lower bound for the uncertainties of two observables in terms of their commutator and their noise correlator (Gottfried, 1966),

$$
(\Delta A)^{2}(\Delta B)^{2} \geqslant \frac{1}{4}\langle\{\hat{A}, \hat{B}\}\rangle^{2}+\frac{1}{4}|\langle[\hat{A}, \hat{B}]\rangle|^{2} .
$$

Here we have assumed $\langle\hat{A}\rangle=\langle\hat{B}\rangle=0$. We now choose the Hermitian operators $\hat{A}$ and $\hat{B}$ to be given by the cosine transforms of $\hat{I}$ and $\hat{F}$, respectively, over a finite time interval $T$,

$$
\begin{aligned}
\hat{A} & \equiv \sqrt{\frac{2}{T}} \int_{-T / 2}^{T / 2} d t \cos (\omega t+\delta) \hat{I}(t), \\
\hat{B} & \equiv \sqrt{\frac{2}{T}} \int_{-T / 2}^{T / 2} d t \cos (\omega t) \hat{F}(t) .
\end{aligned}
$$

Note that we have phase shifted the transform of $\hat{I}$ relative to that of $\hat{F}$ by a phase $\delta$. In the limit $T \rightarrow \infty$ we find, at any finite frequency $\omega \neq 0$

$$
\begin{aligned}
(\Delta A)^{2}= & \bar{S}_{I I}[\omega], \quad(\Delta B)^{2}=\bar{S}_{F F}[\omega], \\
\langle\{\hat{A}, \hat{B}\}\rangle & =2 \operatorname{Re} e^{i \delta} \bar{S}_{I F}[\omega], \\
\langle[\hat{A}, \hat{B}]\rangle & =\int_{-\infty}^{+\infty} d t \cos (\omega t+\delta)\langle[\hat{I}(t), \hat{F}(0)]\rangle \\
& =i \hbar \operatorname{Re}\left[e^{i \delta}\left(\chi_{I F}[\omega]-\left(\chi_{F I}[\omega]\right)^{*}\right)\right] .
\end{aligned}
$$

In the last equality, we have simply made use of the Kubo formula definitions of the gain and reverse gain [cf. Eqs. (4.3) and (4.6)]. As a consequence of Eqs. (4.15a), (4.15b), and (4.15c), the Heisenberg uncertainty relation (4.13) directly yields

$$
\begin{aligned}
\bar{S}_{I I}[\omega] \bar{S}_{F F}[\omega] \geqslant & \left\{\operatorname{Re}\left(e^{i \delta} \bar{S}_{I F}[\omega]\right)\right\}^{2}+\frac{\hbar^{2}}{4}\left[\operatorname { R e } e ^ { i \delta } \left(\chi_{I F}[\omega]\right.\right. \\
& \left.\left.-\left(\chi_{F I}[\omega]\right)^{*}\right)\right]^{2} .
\end{aligned}
$$

Maximizing the RHS of this inequality over $\delta$ then yields the general quantum noise constraint of Eq. (4.11).

With this derivation, we can now interpret the quantum noise constraint Eq. (4.11) as stating that the noise at a given frequency given frequency in two observables
$\hat{I}$ and $\hat{F}$ is bounded by the value of their commutator at that frequency. The fact that $\hat{I}$ and $\hat{F}$ do not commute is necessary for the existence of linear response (gain) from the detector, but also means that the noise in both $\hat{I}$ and $\hat{F}$ cannot be arbitrarily small. A more detailed derivation, yielding additional important insights, is described in Appendix I.1.

Given the quantum noise constraint of Eq. (4.11), we can now very naturally define a quantum-ideal detector (at a given frequency $\omega$ ) as one which minimizes the left-hand side (LHS) of Eq. (4.11) — a quantum-ideal detector has a minimal amount of noise at frequency $\omega$. We are often interested in the ideal case where there is no reverse gain (i.e., measuring $\hat{I}$ does not result in additional back-action noise in $\hat{F}$ ); the condition to have a quantum-limited detector thus becomes

$$
\begin{aligned}
\bar{S}_{I I}[\omega] \bar{S}_{F F}[\omega]-\left|\bar{S}_{I F}[\omega]\right|^{2} \\
=\left|\frac{\hbar \chi_{I F}[\omega]}{2}\right|^{2}\left(1+\Delta\left[\frac{\bar{S}_{I F}[\omega]}{\hbar \chi_{I F}[\omega] / 2}\right]\right),
\end{aligned}
$$

where $\Delta[z]$ is given in Eq. (4.12). Again, as discussed below, in most cases of interest (e.g., zero frequency and/or large amplifier power gain) the last term on the RHS will vanish. In the following, we demonstrate that the ideal noise requirement of Eq. (4.17) is necessary in order to achieve the quantum limit on QND detection of a qubit, or on the added noise of a linear amplifier.

Before leaving our general discussion of the quantum noise constraint, it is worth emphasizing that achieving Eq. (4.17) places a strong constraint on the properties of the detector. In particular, there must exist a tight connection between the input and output ports of the detector-in a certain restricted sense, the operators $\hat{I}$ and $\hat{F}$ must be proportional to one another [see Eq. (I13) in Appendix I.1]. As is discussed in Appendix I.1, this proportionality immediately tells us that a quantumideal detector cannot be in equilibrium. The proportionality exhibited by a quantum-ideal detector is parametrized by a single complex-valued number $\alpha[\omega]$, whose magnitude is given by

$$
|\alpha[\omega]|^{2}=\bar{S}_{I I}[\omega] / \bar{S}_{F F}[\omega] .
$$

While this proportionality requirement may seem purely formal, it does have a simple heuristic interpretation; as discussed by Clerk et al. (2003), it may be viewed as a formal expression of the principle that a quantumlimited detector must not contain any wasted information (cf. Sec. III.B.2).

\section{Evading the detector quantum noise inequality}

We now turn to situations where the RHS of Eq. (4.11) vanishes, implying that there is no additional quantum constraint on the noise of our detector beyond what exists classically. In such situations, one could have a detector with perfectly correlated back-action and out- 
put noises (i.e., $\bar{S}_{F F} \bar{S}_{I I}=\left|\bar{S}_{I F}\right|^{2}$ ), or even with a vanishing back-action $\bar{S}_{F F}=0$. Perhaps not surprisingly, these situations are not of much utility. As we now show, in cases where the RHS of Eq. (4.11) vanishes, the detector may be low noise, but will necessarily be deficient in another important regard: it will not be good enough that we can ignore the extra noise associated with the measurement of the detector output $\hat{I}$. As discussed, the reading out of $\hat{I}$ will invariably involve coupling the detector output to some other physical system. In the ideal case, this coupling will not generate any additional back-action on the system coupled to the detector's input port. In addition, the signal at the detector output should be large enough that any noise introduced in measuring $\hat{I}$ is negligible; we already came across this idea in our discussion of the resonant cavity detector [see discussion following Eq. (3.60)]. This means that we need our detector to truly amplify the input signal, not simply reproduce it at the output with no gain in energy. As now shown, a detector that evades the quantum constraint of Eq. (4.11) by making the RHS of the inequality zero will necessarily fail in one or both of the above requirements.

The most obvious case where the quantum noise constraint vanishes is for a detector which has equal forward and reverse gains, $\chi_{F I}=\chi_{I F}^{*}$. As mentioned, this relation will necessarily hold if the detector is time-reversal symmetric and in equilibrium, and $\hat{I}$ and $\hat{F}$ have the same parity under time reversal. In this case, the relatively large reverse gain implies that in the analysis of a given measurement task, it is not sufficient to just consider the noise of the detector: one must necessarily also consider the noise associated with whatever system is coupled to $\hat{I}$ to read out the detector output, as this noise will be fed back to the detector input port, causing additional backaction; we give an explicit example of this in the next subsection, where we discuss QND qubit detection. Even more problematically, when $\chi_{F I}=\chi_{I F}^{*}$, there is never any amplification by the detector. As discussed in Sec. V.E.3, the proper metric of the detector's ability to amplify is its dimensionless power gain: What is the power supplied at the output of the detector versus the amount of power drawn at the input from the signal source? When $\chi_{F I}=\chi_{I F}^{*}$, one has negative feedback, with the result that the power gain cannot be larger than 1 [see Eq. (5.53)]. There is thus no amplification when $\chi_{F I}=\chi_{I F}^{*}$. Further, if one also insists that the noise constraint of Eq. (4.11) is optimized, then one finds the power gain must be exactly 1 ; this is explicitly demonstrated in Appendix I.2. The detector thus will simply act as a transducer, reproducing the input signal at the output without any increase in energy. We have here a specific example of a more general idea that will be discussed in Sec. V: if a detector acts only as a transducer, it need not add any noise.

At finite frequencies, there is a second way to make the RHS of the quantum noise constraint of Eq. (4.11) vanish: one needs the quantity $\bar{S}_{I F}[\omega] / \tilde{\chi}_{I F}$ to be purely imaginary, and larger in magnitude than $\hbar / 2$. In this case, it would again be possible to have the LHS of the noise constraint of Eq. (4.11) equal to 0. However, one again finds that in such a case the dimensionless power gain of the detector is at most equal to 1 ; it thus does not amplify. This is shown explicitly in Appendix I.2. An important related statement is that a quantum-limited detector with a large power gain must have the quantity $\bar{S}_{I F} / \chi_{I F}$ be real. Thus, at the quantum limit, correlations between the back-action force and the intrinsic output noise fluctuations must have the same phase as the gain $\chi_{I F}$. As discussed in Sec. V.F, this requirement can be interpreted in terms of the principle of no wasted information introduced in Sec. III.B.2.

\section{B. Quantum limit on QND detection of a qubit}

In Sec. III.B, we discussed the quantum limit on QND qubit detection in the specific context of a resonant cavity detector. We now show how the full quantum noise constraint of Eq. (4.11) directly leads to this quantum limit for an arbitrary weakly coupled detector. Similar to Sec. III.B, we couple the input operator of our generic linear-response detector to the $\hat{\sigma}_{z}$ operator of the qubit we wish to measure [i.e., we take $\hat{x}=\hat{\sigma}_{z}$ in Eq. (4.1)]; we also consider the QND regime, where $\hat{\sigma}_{z}$ commutes with the qubit Hamiltonian. As we saw in Sec. III.B, the quantum limit in this case involves the inequality $\Gamma_{\text {meas }}$ $\leqslant \Gamma_{\varphi}$, where $\Gamma_{\text {meas }}$ is the measurement rate and $\Gamma_{\varphi}$ is the back-action dephasing rate. For the latter quantity, we can directly use the results of our calculation for the cavity system, where we found the dephasing rate was set by the zero-frequency noise in the cavity photon number [see Eq. (3.27)]. In complete analogy, the backaction dephasing rate will be determined here by the zero-frequency noise in the input operator $\hat{F}$ of our detector,

$$
\Gamma_{\varphi}=\left(2 A^{2} / \hbar^{2}\right) \bar{S}_{F F}
$$

We omit frequency arguments in this section, as it is always the zero-frequency susceptibilities and spectral densities that appear.

The measurement rate (the rate at which information on the state of qubit is acquired) is also defined in complete analogy to what was done for the cavity detector. We imagine we turn the measurement on at $t=0$ and start to integrate up the output $I(t)$ of our detector,

$$
\hat{m}(t)=\int_{0}^{t} d t^{\prime} \hat{I}\left(t^{\prime}\right) .
$$

The probability distribution of the integrated output $\hat{m}(t)$ will depend on the state of the qubit; for long times, we may approximate the distribution corresponding to each qubit state as being Gaussian. Noting that we have chosen $\hat{I}$ so that its expectation vanishes at zero coupling, the average value of $\langle\hat{m}(t)\rangle$ corresponding to each qubit state is (in the long-time limit of interest) 


$$
\langle\hat{m}(t)\rangle_{\uparrow}=A \chi_{I F} t, \quad\langle\hat{m}(t)\rangle_{\downarrow}=-A \chi_{I F} t .
$$

The variance of both distributions is, to leading order, independent of the qubit state,

$$
\left\langle\hat{m}^{2}(t)\right\rangle_{\uparrow / \downarrow}-\langle\hat{m}(t)\rangle_{\uparrow / \downarrow}^{2} \equiv\left\langle\left\langle\hat{m}^{2}(t)\right\rangle\right\rangle_{\uparrow \uparrow \downarrow}=\bar{S}_{I I} t .
$$

For the last equality above, we have taken the long-time limit, which results in the variance of $\hat{m}$ being determined completely by the zero-frequency output noise $\bar{S}_{I I}[\omega=0]$ of the detector. The assumption here is that, due to the weakness of the measurement, the measurement time $1 / \Gamma_{\text {meas }}$ will be much longer than the autocorrelation time of the detector's noise.

We can now define the measurement rate, in complete analogy to the cavity detector of the previous section [cf. Eq. (3.23)], by how quickly the resolving power of the measurement grows, ${ }^{7}$

$$
\frac{1}{4}\left[\langle\hat{m}(t)\rangle_{\uparrow}-\langle\hat{m}(t)\rangle_{\downarrow}\right]^{2} /\left[\left\langle\left\langle\hat{m}^{2}(t)\right\rangle\right\rangle_{\uparrow}+\left\langle\left\langle\hat{m}^{2}(t)\right\rangle\right\rangle_{\downarrow}\right] \equiv \Gamma_{\text {meas }} t .
$$

This yields

$$
\Gamma_{\text {meas }}=A^{2}\left(\chi_{I F}\right)^{2} / 2 \bar{S}_{I I} .
$$

Putting this all together, we find that the "efficiency" ratio $\eta=\Gamma_{\text {meas }} / \Gamma_{\varphi}$ is given by

$$
\eta \equiv \Gamma_{\text {meas }} / \Gamma_{\varphi}=\hbar^{2}\left(\chi_{I F}\right)^{2} / 4 \bar{S}_{I I} \bar{S}_{F F} .
$$

In the case where our detector has a vanishing reverse gain (i.e., $\chi_{F I}=0$ ), the quantum-limit bound $\eta \leqslant 1$ follows immediately from the quantum noise constraint of Eq. (4.11). We thus see that achieving the quantum limit for QND qubit detection requires both a detector with quantum-ideal noise properties, as defined by Eq. (4.17), and a detector with a vanishing noise cross correlator: $\bar{S}_{I F}=0$.

If in contrast $\chi_{F I} \neq 0$, it would seem that it is possible to have $\eta \geqslant 1$. This is of course an invalid inference: as discussed, $\chi_{F I} \neq 0$ implies that we must necessarily consider the effects of extra noise injected into the detector's output port when one measures $\hat{I}$, as the reverse gain will bring this noise back to the qubit, causing extra dephasing. The result is that one can do no better than $\eta=1$. To see this explicitly, consider the extreme case $\chi_{I F}=\chi_{F I}$ and $\bar{S}_{I I}=\bar{S}_{F F}=0$, and suppose we use a second detector to read out the output $\hat{I}$ of the first detector. This second detector has input and output operators $\hat{F}_{2}$, $\hat{I}_{2}$; we also take it to have a vanishing reverse gain, so that we do not have to also worry about how its output is read out. Coupling of the detectors linearly in the standard way (i.e., $H_{\mathrm{int}, 2}=\hat{I} \hat{F}_{2}$ ), gives the overall gain of

\footnotetext{
${ }^{7}$ The factor of $1 / 4$ here is purely chosen for convenience; we are defining the measurement rate based on the informationtheoretic definition given in Appendix F. This factor of 4 is consistent with the definition used in the cavity system.
}

the two detectors in series as $\chi_{I_{2} F_{2}} \chi_{I F}$, while the backaction driving the qubit dephasing is described by the spectral density $\left(\chi_{F I}\right)^{2} S_{F_{2} F_{2}}$. Using the fact that our second detector must itself satisfy the quantum noise inequality, we have

$$
\left[\left(\chi_{F I}\right)^{2} \bar{S}_{F_{2} F_{2}}\right] \bar{S}_{I_{2} I_{2}} \geqslant\left(\hbar^{2} / 4\right)\left(\chi_{I_{2} F_{2}} \chi_{I F}\right)^{2} .
$$

Thus, the overall chain of detectors satisfies the usual zero-reverse-gain quantum noise inequality, implying that we still have $\eta \leqslant 1$.

\section{QUANTUM LIMIT ON LINEAR AMPLIFIERS AND POSITION DETECTORS}

In the previous section we established the fundamental quantum constraint on the noise of any system capable of acting as a linear detector; we further showed that this quantum noise constraint directly leads to the quantum limit on nondemolition qubit detection using a weakly coupled detector. In this section, we turn to the more general situation where our detector is a phasepreserving quantum linear amplifier: the input to the detector is described by some time-dependent operator $\hat{x}(t)$, which we wish to have amplified at the output of our detector. As we see, the quantum limit in this case is a limit on how small one can make the noise added by the amplifier to the signal. The discussion in this section both furthers and generalizes the heuristic discussion of position detection using a cavity detector presented in Sec. III.B.

In this section, we start by presenting a heuristic discussion of quantum constraints on amplification. We then demonstrate explicitly how the previously discussed quantum noise constraint leads directly to the quantum limit on the added noise of a phase-preserving linear amplifier; we examine the cases of both a generic linear position detector and a generic voltage amplifier, following the approach outlined by Clerk (2004). We also spend time explicitly connecting the linear-response approach used here to the bosonic scattering formulation of the quantum limit favored by the quantum optics community (Haus and Mullen, 1962; Caves, 1982; Grassia, 1998; Courty et al., 1999), paying particular attention to the case of a two-port scattering amplifier. We will see that there are some important subtleties involved in converting between the two approaches. In particular, there exists a crucial difference between the case where the input signal is tightly coupled to the input of the amplifier (the case usually considered in the quantum optics community), versus the case where, as in an ideal opamp, the input signal is only weakly coupled to the input of the amplifier (the case usually considered in the solid state community).

\section{A. Preliminaries on amplification}

What exactly does one mean by amplification? As we will see (Sec. V.E.3), a precise definition requires that the energy provided at the output of the amplifier be much 
larger than the energy drawn at the input of the amplifier-the power gain of the amplifier must be larger than 1. For the moment, however, we work with the cruder definition that amplification involves making some time-dependent signal larger. To set the stage, we first consider an extremely simple classical analog of a linear amplifier. Imagine that the "signal" we wish to amplify is the coordinate $x(t)$ of a harmonic oscillator; we can write this signal as

$$
x(t)=x(0) \cos \left(\omega_{S} t\right)+\left[p(0) / M \omega_{S}\right] \sin \left(\omega_{S} t\right) .
$$

Our signal has two quadrature amplitudes, i.e., the amplitude of the cosine and sine components of $x(t)$. To amplify this signal, we start at $t=0$ to parametrically drive the oscillator by changing its frequency $\omega_{S}$ periodically in time: $\omega_{S}(t)=\omega_{0}+\delta \omega \sin \left(\omega_{P} t\right)$, where we assume $\delta \omega \ll \omega_{0}$. The well-known physical example is a swing whose motion is being excited by effectively changing the length of the pendulum at the right frequency and phase. For a "pump frequency" $\omega_{P}$ equalling twice the "signal frequency," $\omega_{P}=2 \omega_{S}$, the resulting dynamics will lead to an amplification of the initial oscillator position, with the energy provided by the external driving,

$$
x(t)=x(0) e^{\lambda t} \cos \left(\omega_{S} t\right)+\left[p(0) / M \omega_{S}\right] e^{-\lambda t} \sin \left(\omega_{S} t\right) .
$$

Thus, one of the quadratures is amplified exponentially, at a rate $\lambda=\delta \omega / 2$, while the other one decays. In a quantum-mechanical description, this produces a squeezed state out of an initial coherent state. Such a system is called a "degenerate parametric amplifier," and we discuss its quantum dynamics in Sec. V.H. We see that such an amplifier, which amplifies only a single quadrature, is not required quantum mechanically to add any noise (Caves et al., 1980; Caves, 1982; Braginsky and Khalili, 1992).

Can we now change this parametric amplification scheme slightly in order to make both signal quadratures grow with time? It turns out that this is impossible as long as we restrict ourselves to a driven system with a single degree of freedom. The reason in classical mechanics is that Liouville's theorem requires phase-space volume to be conserved during motion. More formally, this is related to the conservation of Poisson brackets, or in quantum mechanics to the conservation of commutation relations. Nevertheless, it is certainly desirable to have an amplifier that acts equally on both quadratures (a so-called phase-preserving or phase-insensitive amplifier), since the signal's phase is often not known beforehand. The way around the restriction created by Liouville's theorem is to add more degrees of freedom, such that the phase-space volume can expand in both quadratures (i.e., position and momentum) of the interesting signal degree of freedom, while being compressed in other directions. This is achieved most easily by coupling the signal oscillator to another oscillator, the "idler mode." The external driving now modulates the coupling between these oscillators, at a frequency that has to equal the sum of the oscillators' frequencies. The resulting scheme is called a phase-preserving nondegenerate parametric amplifier (see Sec. V.C).

Crucially, there is a price to pay for the introduction of an extra degree of freedom: there will be noise associated with the idler oscillator, and this noise will contribute to the noise in the output of the amplifier. Classically, one could make the noise associated with the idler oscillator arbitrarily small by simply cooling it to zero temperature. This is not possible quantum mechanically; there are always zero-point fluctuations of the idler oscillator to contend with. It is this noise which sets a fundamental quantum limit for the operation of the amplifier. We thus have a heuristic accounting for the existence of a quantum limit on the added noise of a phase-preserving linear amplifier: one needs extra degrees of freedom to amplify both signal quadratures, and such extra degrees of freedom invariably have noise associated with them.

\section{B. Standard Haus-Caves derivation of the quantum limit on a bosonic amplifier}

We now make the ideas of the previous section more precise by sketching the standard derivation of the quantum limit on the noise added by a phase-preserving amplifier. This derivation is originally due to Haus and Mullen (1962), and was both clarified and extended by Caves (1982); the amplifier quantum limit was also motivated in a slightly different manner by Heffner (1962). ${ }^{8}$ While extremely compact, the Haus-Caves derivation can lead to confusion when improperly applied; we discuss this in Sec. V.D, as well as in Sec. VI, where we apply this argument carefully to the important case of a two-port quantum voltage amplifier and discuss the connection to the general linear-response formulation of Sec. IV.

The starting assumption of this derivation is that both the input and output ports of the amplifier can be described by sets of bosonic modes. If we focus on a narrow bandwidth centered on frequency $\omega$, we can describe a classical signal $E(t)$ in terms of a complex number $a$ defining the amplitude and phase of the signal (or equivalently the two quadrature amplitudes) (Haus and Mullen, 1962; Haus, 2000)

$$
E(t) \propto i\left[a e^{-i \omega t}-a^{*} e^{+i \omega t}\right] .
$$

In the quantum case, the two signal quadratures of $E(t)$ [i.e., the real and imaginary parts of $a(t)$ ] cannot be measured simultaneously because they are canonically conjugate; this is in complete analogy to a harmonic oscillator [see Eq. (3.48)]. As a result $a, a^{*}$ must be elevated to the status of photon ladder operators: $a \rightarrow \hat{a}, a^{*} \rightarrow a^{\dagger}$.

Consider the simplest case where there is only a single mode at both the input and output, with corresponding

\footnotetext{
${ }^{8}$ Note that Caves (1982) provided a discussion of why the derivation of the amplifier quantum limit given by Heffner (1962) is not rigorously correct.
} 
operators $\hat{a}$ and $\hat{b} .{ }^{9}$ It follows that the input signal into the amplifier is described by the expectation value $\langle\hat{a}\rangle$, while the output signal is described by $\langle\hat{b}\rangle$. Correspondingly, the symmetrized noise in both these quantities is described by

$$
(\Delta a)^{2} \equiv \frac{1}{2}\left\langle\left\{\hat{a}, \hat{a}^{\dagger}\right\}\right\rangle-|\langle\hat{a}\rangle|^{2},
$$

with an analogous definition for $(\Delta b)^{2}$.

To derive a quantum limit on the added noise of the amplifier, one uses two simple facts. First, both the input and the output operators must satisfy the usual commutation relations

$$
\left[\hat{a}, \hat{a}^{\dagger}\right]=1, \quad\left[\hat{b}, \hat{b}^{\dagger}\right]=1 .
$$

Second, the linearity of the amplifier and the fact that it is phase preserving (i.e., both signal quadratures are amplified the same way) implies a simple relation between the output operator $\hat{b}$ and the input operator $\hat{a}$,

$$
\hat{b}=\sqrt{G} \hat{a}, \quad \hat{b}^{\dagger}=\sqrt{G} \hat{a}^{\dagger},
$$

where $G$ is the dimensionless photon-number gain of the amplifier. It is clear, however, that this expression cannot possibly be correct as written because it violates the fundamental bosonic commutation relation $\left[\hat{b}, \hat{b}^{\dagger}\right]=1$. We are therefore forced to write

$$
\hat{b}=\sqrt{G} \hat{a}+\hat{\mathcal{F}}, \quad \hat{b}^{\dagger}=\sqrt{G} \hat{a}^{\dagger}+\hat{\mathcal{F}}^{\dagger},
$$

where $\hat{\mathcal{F}}$ is an operator representing additional noise added by the amplifier. Based on the discussion of the previous section, we can anticipate what $\hat{\mathcal{F}}$ represents: it is noise associated with the additional degrees of freedom that must invariably be present in a phasepreserving amplifier.

As $\hat{\mathcal{F}}$ represents noise, it has a vanishing expectation value; in addition, one also assumes that this noise is uncorrelated with the input signal, implying $[\hat{\mathcal{F}}, \hat{a}]$ $=\left[\hat{\mathcal{F}}, \hat{a}^{\dagger}\right]=0$ and $\langle\hat{\mathcal{F}} \hat{a}\rangle=\left\langle\hat{\mathcal{F}} \hat{a}^{\dagger}\right\rangle=0$. Insisting that $\left[\hat{b}, \hat{b}^{\dagger}\right]=1$ thus yields

$$
\left[\hat{\mathcal{F}}, \hat{\mathcal{F}}^{\dagger}\right]=1-G \text {. }
$$

The question now becomes how small can we make the noise described by $\hat{\mathcal{F}}$ ? From Eqs. (5.7), the noise at the amplifier output $\Delta b$ is given by

$$
\begin{aligned}
(\Delta b)^{2} & =G(\Delta a)^{2}+\frac{1}{2}\left\langle\left\{\hat{\mathcal{F}}, \hat{\mathcal{F}}^{\dagger}\right\}\right\rangle \geqslant G(\Delta a)^{2}+\frac{1}{2}\left|\left\langle\left[\hat{\mathcal{F}}, \hat{\mathcal{F}}^{\dagger}\right]\right\rangle\right| \\
& \geqslant G(\Delta a)^{2}+|G-1| / 2 .
\end{aligned}
$$

We have used here a standard inequality to bound the expectation of $\left\{\hat{\mathcal{F}}, \hat{\mathcal{F}}^{\dagger}\right\}$. The first term here is simply the

\footnotetext{
${ }^{9}$ To relate this to the linear-response detector of Sec. IV.A, one could naively write $\hat{x}$, the operator carrying the input signal as, $\hat{x}=\hat{a}+\hat{a}^{\dagger}$, and the output operator $\hat{I}$ as, $\hat{I}=\hat{b}+\hat{b}^{\dagger}$ (we discuss how to make this correspondence in Sec. VI).
}

amplified noise of the input, while the second term represents the noise added by the amplifier. Note that if there is no amplification (i.e., $G=1$ ), there need not be any added noise. However, in the more relevant case of large amplification $(G \gg 1)$, the added noise cannot vanish. It is useful to express the noise at the output as an equivalent noise at the input by simply dividing out the photon gain $G$. Taking the large- $G$ limit, we have

$$
(\Delta b)^{2} / G \geqslant(\Delta a)^{2}+\frac{1}{2} \text {. }
$$

Thus, we have a simple demonstration that an amplifier with a large photon gain must add at least half a quantum of noise to the input signal. Equivalently, the minimum value of the added noise is equal to the zero-point noise associated with the input mode; the total output noise (referred to the input) is at least twice the zeropoint input noise. Note that both these conclusions are identical to what we found in our analysis of the resonant cavity position detector in Sec. III.B.3. We discuss later how this conclusion can also be reached using the general linear-response language of Sec. IV (cf. Secs. V.E and V.F).

As discussed, the added noise operator $\mathcal{F}$ is associated with additional degrees of freedom (beyond input and output modes) necessary for phase-preserving amplification. To see this more concretely, note that every linear amplifier is inevitably a nonlinear system consisting of an energy source and a "spigot" controlled by the input signal, which redirects the energy source partly to the output channel and partly to some other channel(s). Hence there are inevitably other degrees of freedom involved in the amplification process beyond the input and output channels. An explicit example is the quantum parametric amplifier, discussed in the next subsection. Further insights into amplifier-added noise and its connection to the fluctuation-dissipation theorem can be obtained by considering a simple model where a transmission line is terminated by an effective negative impedance; we discuss this model in Appendix C.4.

To see explicitly the role of the additional degrees of freedom, note first that for $G>1$ the RHS of Eq. (5.8) is negative. Hence the simplest possible form for the added noise is

$$
\hat{\mathcal{F}}=\sqrt{G-1} \hat{d}^{\dagger}, \quad \hat{\mathcal{F}}^{\dagger}=\sqrt{G-1} \hat{d},
$$

where $\hat{d}$ and $\hat{d}^{\dagger}$ represent a single additional mode of the system. This is the minimum number of additional degrees of freedom that must inevitably be involved in the amplification process. Note that for this case the inequality in Eq. (5.9) is satisfied as an equality, and the added noise takes on its minimum possible value. If instead we have, say, two additional modes (coupled inequivalently)

$$
\hat{\mathcal{F}}=\sqrt{G-1}\left(\cosh \theta \hat{d}_{1}^{\dagger}+\sinh \theta \hat{d}_{2}\right),
$$

it is straightforward to show that the added noise is inevitably larger than the minimum. This again can be interpreted in terms of wasted information, as the extra degrees of freedom are not being monitored as part of 


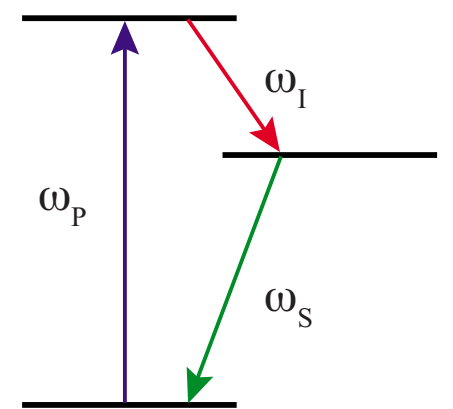

FIG. 8. (Color online) Energy level scheme of the nondegenerate (phase-preserving) parametric oscillator.

the measurement process and so information is being lost.

\section{Nondegenerate parametric amplifier}

\section{Gain and added noise}

Before we start our discussion of the connection of the Haus-Caves formulation of the quantum limit to the general linear-response approach of Sec. IV, it is useful to consider a specific example. To that end, we analyze here a nondegenerate parametric amplifier, a linear phase-preserving amplifier which reaches the quantum limit on its added noise (Louisell et al., 1961; Gordon et al., 1963; Mollow and Glauber, 1967a, 1967b) and directly realizes the ideas of the previous subsection. One possible realization (Yurke et al., 1989) is a cavity with three internal resonances that are coupled together by a nonlinear element (such as a Josephson junction) whose symmetry permits three-wave mixing. The three modes are called the pump, idler, and signal and their energy level structure (shown in Fig. 8) obeys $\omega_{P}=\omega_{I}+\omega_{S}$. The system Hamiltonian is then

$$
\begin{aligned}
\hat{H}_{\mathrm{sys}}= & \hbar\left(\omega_{P} \hat{a}_{P}^{\dagger} \hat{a}_{P}+\omega_{I} \hat{a}_{I}^{\dagger} \hat{a}_{I}+\omega_{S} \hat{a}_{S}^{\dagger} \hat{a}_{S}\right)+i \hbar \eta\left(\hat{a}_{S}^{\dagger} \hat{a}_{I}^{\dagger} \hat{a}_{P}\right. \\
& \left.-\hat{a}_{S} \hat{a}_{I} \hat{a}_{P}^{\dagger}\right) .
\end{aligned}
$$

We have made the rotating wave approximation in the three-wave mixing term, and without loss of generality we take the nonlinear susceptibility $\eta$ to be real and positive. The system is driven at the pump frequency and the three-wave mixing term permits a single pump photon to split into an idler photon and a signal photon. This process is stimulated by signal photons already present and leads to gain. A typical mode of operation would be the negative-resistance reflection mode in which the input signal is reflected from a nonlinear cavity and the reflected beam extracted using a circulator (Yurke et al., 1989; Bergeal et al., 2008).

The nonlinear equations of motion (EOMs) become tractable if we assume the pump has large amplitude and can be treated classically by making the substitution

$$
\hat{a}_{P}=\psi_{P} e^{-i \omega_{P} t}=\psi_{P} e^{-i\left(\omega_{\mathrm{I}}+\omega_{S}\right) t},
$$

where without loss of generality we take $\psi_{P}$ to be real and positive. We note here the important point that if this approximation were not valid, then our amplifier would in any case not be the linear amplifier we seek. With this approximation we can hereafter ignore the dynamics of the pump degree of freedom and deal with the reduced system Hamiltonian

$$
\begin{aligned}
\hat{H}_{\mathrm{sys}}= & \hbar\left(\omega_{\mathrm{I}} \hat{a}_{\mathrm{I}}^{\dagger} \hat{a}_{\mathrm{I}}+\omega_{S} \hat{a}_{S}^{\dagger} \hat{a}_{S}\right)+i \hbar \lambda\left(\hat{a}_{S}^{\dagger} \hat{a}_{\mathrm{I}}^{\dagger} e^{-i\left(\omega_{\mathrm{I}}+\omega_{S}\right) t}\right. \\
& \left.-\hat{a}_{S} \hat{a}_{\mathrm{I}} e^{+i\left(\omega_{\mathrm{I}}+\omega_{S}\right) t}\right),
\end{aligned}
$$

where $\lambda \equiv \eta \psi_{P}$. Transforming to the interaction representation we are left with the following timeindependent quadratic Hamiltonian for the system

$$
\hat{V}_{\text {sys }}=i \hbar \lambda\left(\hat{a}_{S}^{\dagger} \hat{a}_{\mathrm{I}}^{\dagger}-\hat{a}_{S} \hat{a}_{\mathrm{I}}\right) \text {. }
$$

To get some intuitive understanding of the physics, we temporarily ignore the damping of the cavity modes that would result from their coupling to modes outside the cavity. We now have a pair of coupled EOMs for the two modes

$$
\dot{\hat{a}}_{S}=+\lambda \hat{a}_{\mathrm{I}}^{\dagger}, \quad \dot{\hat{a}}_{\mathrm{I}}^{\dagger}=+\lambda \hat{a}_{S},
$$

for which the solutions are

$$
\begin{aligned}
& \hat{a}_{S}(t)=\cosh (\lambda t) \hat{a}_{S}(0)+\sinh (\lambda t) \hat{a}_{\mathrm{I}}^{\dagger}(0), \\
& \hat{a}_{\mathrm{I}}^{\dagger}(t)=\sinh (\lambda t) \hat{a}_{S}(0)+\cosh (\lambda t) \hat{a}_{\mathrm{I}}^{\dagger}(0) .
\end{aligned}
$$

We see that the amplitude in the signal channel grows exponentially in time and that the effect of the time evolution is to perform a simple unitary transformation which mixes $\hat{a}_{S}$ with $\hat{a}_{\mathrm{I}}^{\dagger}$ in such a way as to preserve the commutation relations. Note the close connection with the form found from general arguments in Eqs. (5.7)-(5.11)

We may now tackle the full system which includes the coupling between the cavity modes and modes external to the cavity. Such a coupling is of course necessary in order to feed the input signal into the cavity, as well as extract the amplified output signal. It will also result in the damping of the cavity modes, which will cut off the exponential growth found above and yield a fixed amplitude gain. We present the main results in this section, relegating details to how one treats the bath modes [socalled input-output theory (Walls and Milburn, 1994)] to Appendix E. Working in the standard Markovian limit, we obtain the following EOMs in the interaction representation:

$$
\begin{aligned}
& \dot{\hat{a}}_{S}=-\left(\kappa_{S} / 2\right) \hat{a}_{S}+\lambda \hat{a}_{\mathrm{I}}^{\dagger}-\sqrt{\kappa_{S}} \hat{b}_{S, \text { in }}, \\
& \dot{\hat{a}}_{\mathrm{I}}^{\dagger}=-\left(\kappa_{\mathrm{I}} / 2\right) \hat{a}_{\mathrm{I}}^{\dagger}+\lambda \hat{a}_{S}-\sqrt{\kappa_{\mathrm{I}}} \hat{b}_{\mathrm{I}, \text { in }}^{\dagger} .
\end{aligned}
$$

Here $\kappa_{S}$ and $\kappa_{\mathrm{I}}$ are the respective damping rates of the cavity signal mode and the idler mode. The coupling to extra-cavity modes also lets signals and noise enter the cavity from the baths: this is described by the bosonic operators $\hat{b}_{S \text {,in }}$ and $\hat{b}_{\text {I,in }}$ which drive the signal and idler modes, respectively. $\hat{b}_{S \text {,in }}$ describes both the input signal to be amplified and vacuum noise entering from the bath 
coupled to the signal mode, whereas $\hat{b}_{\mathrm{I}, \text { in }}$ simply describes vacuum noise. ${ }^{10}$

Let us fix our attention on signals inside a frequency window $\delta \omega$ centered on $\omega_{S}$ (hence zero frequency in the interaction representation). For simplicity, we first consider the case where the signal bandwidth $\delta \omega$ is almost infinitely narrow (i.e., much smaller than the damping rate of the cavity modes). It then suffices to find the steady state solution of these EOMs,

$$
\begin{aligned}
& \hat{a}_{S}=\left(2 \lambda / \kappa_{S}\right) \hat{a}_{\mathrm{I}}^{\dagger}-\left(2 / \sqrt{\kappa_{S}}\right) \hat{b}_{S, \text { in }}, \\
& \hat{a}_{\mathrm{I}}^{\dagger}=\left(2 \lambda / \kappa_{\mathrm{I}}\right) \hat{a}_{S}-\left(2 / \sqrt{\kappa_{\mathrm{I}}}\right) \hat{b}_{\mathrm{I}, \mathrm{in}}^{\dagger} .
\end{aligned}
$$

The output signal of the nondegenerate paramp is the signal leaving the cavity signal mode and entering the external bath modes; it is described by an operator $\hat{b}_{S \text {,out }}$. The standard input-output theory treatment of the extra-cavity modes (Walls and Milburn, 1994), presented in Appendix E, yields the simple relation [cf. Eq. (E37)]

$$
\hat{b}_{S, \text { out }}=\hat{b}_{S, \text { in }}+\sqrt{\kappa_{S}} \hat{a}_{S} .
$$

The first term corresponds to the reflection of the signal and noise incident on the cavity from the bath, while the second term corresponds to radiation from the cavity mode into the bath. Using this, we find that the output signal from the cavity is given by

$$
\hat{b}_{S, \text { out }}=\frac{Q^{2}+1}{Q^{2}-1} \hat{b}_{S, \text { in }}+\frac{2 Q}{Q^{2}-1} \hat{b}_{\mathrm{I}, \text { in }}^{\dagger},
$$

where $Q \equiv 2 \lambda / \sqrt{\kappa_{\mathrm{I}} \kappa_{S}}$ is proportional to the pump amplitude and inversely proportional to the cavity decay rates. We have to require $Q^{2}<1$ to make sure that the parametric amplifier does not settle into self-sustained oscillations, i.e., it works below threshold. Under that condition, we can define the photon-number gain $G_{0}$ via

$$
-\sqrt{G_{0}}=\left(Q^{2}+1\right) /\left(Q^{2}-1\right),
$$

such that

$$
\hat{b}_{S, \text { out }}=-\sqrt{G_{0}} \hat{b}_{S, \text { in }}-\sqrt{G_{0}-1} \hat{b}_{\mathrm{I}, \text { in }}^{\dagger} .
$$

In the ideal case, the noise associated with $\hat{b}_{\mathrm{I} \text {,in }}, \hat{b}_{S \text {, in }}$ is simply vacuum noise. As a result, the input-output relation Eq. (5.25) is precisely of the Haus-Caves form (5.11) for an ideal quantum-limited amplifier. It demonstrates that the nondegenerate parametric amplifier reaches the quantum limit for minimum added noise. In the limit of large gain the output noise (referred to the input) for a vacuum input signal is precisely doubled.

\section{Bandwidth-gain trade-off}

The above results neglected the finite bandwidth $\delta \omega$ of the input signal to the amplifier. The gain $G_{0}$ given in Eq. (5.24) is only the gain at precisely the mean signal

\footnotetext{
${ }^{10}$ Note that the $\hat{b}$ operators are not dimensionless, as $\hat{b}^{\dagger} \hat{b}$ represents a photon flux (see Appendix E).
}

frequency $\omega_{S}$; for a finite bandwidth, we also need to understand how the power gain varies as a function of frequency over the entire signal bandwidth. As we see, a parametric amplifier suffers from the fact that as one increases the overall magnitude of the gain at the center frequency $\omega_{S}$ (e.g., by increasing the pump amplitude), one simultaneously narrows the frequency range over which the gain is appreciable. Heuristically, this is because parametric amplification involves using the pump energy to decrease the damping and hence increase the quality factor of the signal mode resonance. This increase in quality factor leads to amplification, but it also reduces the bandwidth over which $\hat{a}_{S}$ can respond to the input signal $\hat{b}_{S \text {,in }}$.

To deal with a finite signal bandwidth, one simply Fourier transforms Eqs. (5.19). The resulting equations are easily solved and substituted into Eq. (5.22), resulting in a frequency-dependent generalization of the input-output relation given in Eq. (5.25),

$$
\hat{b}_{S, \text { out }}[\omega]=-g[\omega] \hat{b}_{S, \text { in }}[\omega]-g^{\prime}[\omega] \hat{b}_{\mathrm{I}, \mathrm{in}}^{\dagger}[\omega] .
$$

Here $g[\omega]$ is the frequency-dependent gain of the amplifier, and $g^{\prime}[\omega]$ satisfies $\left|g^{\prime}[\omega]\right|^{2}=|g[\omega]|^{2}-1$. In the relevant limit where $G_{0}=|g[0]|^{2} \gg 1$ (i.e., large gain at the signal frequency), one has to a good approximation

$$
g[\omega]=\frac{\sqrt{G_{0}}-i\left[\left(\kappa_{S}-\kappa_{\mathrm{I}}\right) /\left(\kappa_{\mathrm{I}}+\kappa_{S}\right)\right](\omega / D)}{1-i(\omega / D)},
$$

with

$$
D=\frac{1}{\sqrt{G_{0}}} \frac{\kappa_{S} \kappa_{\mathrm{I}}}{\kappa_{S}+\kappa_{\mathrm{I}}} .
$$

As always, we work in an interaction picture where the signal frequency has been shifted to zero. $D$ represents the effective operating bandwidth of the amplifier. Components of the signal with frequencies (in the rotating frame) $|\omega| \ll D$ are strongly amplified, while components with frequencies $|\omega| \gg D$ are not amplified at all, but can in fact be slightly attenuated. As already anticipated, the amplification bandwidth $D$ becomes progressively smaller as the pump power and $G_{0}$ are increased, with the product $\sqrt{G_{0}} D$ remaining constant. In a parametric amplifier increasing the gain via increasing the pump strength comes with a price: the effective operating bandwidth is reduced.

\section{Effective temperature}

Recall that in Sec. II.B we introduced the concept of an effective temperature of a nonequilibrium system, Eq. (2.8). As we will discuss, this concept plays an important role in quantum-limited amplifiers; the degenerate paramp gives us a first example of this. Returning to the behavior of the paramp at the signal frequency, we note that Eq. (5.25) implies that, even for vacuum input to both the signal and idler ports, the output will contain a real photon flux. To quantify this in a simple way, it is 
useful to introduce temporal modes which describe the input and output fields during a particular time interval $[j \Delta t,(j+1) \Delta t]$ (where $j$ is an integer),

$$
\hat{B}_{S, \text { in }, j}=\frac{1}{\sqrt{\Delta t}} \int_{j \Delta t}^{(j+1) \Delta t} d \tau \hat{b}_{S, \text { in }}(\tau),
$$

with the temporal modes $\hat{B}_{S, \text { out }, j}$ and $\hat{B}_{\mathrm{I}, \mathrm{in}, j}$ defined analogously. These temporal modes are discussed in Appendix D.2, where we discuss the windowed Fourier transform [see Eq. (D18)].

With the above definition, we find that the output mode will have a real occupancy even if the input mode is empty,

$$
\begin{aligned}
\bar{n}_{S, \text { out }} & =\left\langle 0\left|\hat{B}_{S, \text { out }, j}^{\dagger} \hat{B}_{S, \text { out }, j}\right| 0\right\rangle \\
& =G_{0}\left\langle 0\left|\hat{B}_{S, \text { in }, j}^{\dagger} \hat{B}_{S, \text { in }, j}\right| 0\right\rangle+\left(G_{0}-1\right)\left\langle 0\left|\hat{B}_{\mathrm{I}, \text { in }, j} \hat{B}_{\mathrm{I}, \text { in }, j}^{\dagger}\right| 0\right\rangle \\
& =G_{0}-1 .
\end{aligned}
$$

The dimensionless mode occupancy $\bar{n}_{S \text {,out }}$ is best thought of as a photon flux per unit bandwidth [see Eq. (D26)]. This photon flux is equivalent to the photon flux that would appear in equilibrium at the very high effective temperature (assuming large gain $G_{0}$ )

$$
T_{\mathrm{eff}} \approx \hbar \omega_{S} G_{0} .
$$

This is an example of a more general principle, discussed in Sec. V.E.4: a high-gain amplifier must have associated with it a large effective temperature scale. Referring this total output noise back to the input, we have (in the limit $G_{0} \gg 1$ )

$$
T_{\mathrm{eff}} / G_{0}=\hbar \omega_{S} / 2+\hbar \omega_{S} / 2=\frac{\hbar \omega_{S}}{2}+T_{N} .
$$

This corresponds to the half photon of vacuum noise associated with the signal source, plus the added noise of a half photon of our phase-preserving amplifier (i.e., the noise temperature $T_{N}$ is equal to its quantum-limited value). Here the added noise is simply the vacuum noise associated with the idler port.

The above argument is merely suggestive that the output noise looks like an effective temperature. In fact, it is possible to show that the photon-number distribution of the output is precisely that of a Bose-Einstein distribution at temperature $T_{\text {eff }}$. From Eq. (5.13) we see that the action of the paramp is to destroy a pump photon and create a pair of new photons, one in the signal channel and one in the idler channel. Using the SU $(1,1)$ symmetry of the quadratic hamiltonian in Eq. (5.16) it is possible to show that, for vacuum input, the output of the paramp is a so-called "two-mode squeezed state" of the form (Caves and Schumaker, 1985; Gerry, 1985; Knight and Buzek, 2004)

$$
\left|\Psi_{\text {out }}\right\rangle=Z^{-1 / 2} e^{\alpha b_{S}^{\dagger} b_{I}^{\dagger}}|0\rangle,
$$

where $\alpha$ is a constant related to the gain and, to simplify the notation, we have dropped the "out" labels on the operators. The normalization constant $Z$ can be worked out by expanding the exponential and using

$$
\left(b_{S}^{\dagger}\right)^{n}|0,0\rangle=\sqrt{n !}|n, 0\rangle
$$

to obtain

$$
\left|\Psi_{\text {out }}\right\rangle=Z^{-1 / 2} \sum_{n=0}^{\infty} \alpha^{n}|n, n\rangle
$$

and hence

$$
Z=1 /\left(1-|\alpha|^{2}\right)
$$

so the state is normalizable only for $|\alpha|^{2}<1$.

Because this output is obtained by unitary evolution from the vacuum input state, the output state is a pure state with zero entropy. In light of this, it is interesting to consider the reduced density matrix obtain by tracing over the idler mode. The pure-state density matrix is

$$
\rho=\left|\Psi_{\text {out }}\right\rangle\left\langle\Psi_{\text {out }}\left|=\sum_{m, n=0}^{\infty}\right| n, n\right\rangle \frac{\alpha^{n} \alpha *^{m}}{Z}\langle m, m| .
$$

If we now trace over the idler mode we are left with the reduced density matrix for the signal channel

$$
\tilde{\rho}_{S}=\operatorname{Tr}_{\text {Idler }}\{\rho\}=\sum_{n_{S}=0}^{\infty}\left|n_{S}\right\rangle \frac{|\alpha|^{2 n_{S}}}{Z}\left\langle n_{S}\right| \equiv \frac{1}{Z} e^{-\beta \hbar \omega_{S} a_{S}^{\dagger} a_{S}},
$$

which is a pure thermal equilibrium distribution with effective Boltzmann factor

$$
e^{-\beta \hbar \omega_{S}}=|\alpha|^{2}<1
$$

The effective temperature can be obtained from the requirement that the signal mode occupancy is $G_{0}-1$,

$$
1 /\left(e^{\beta \hbar \omega_{S}}-1\right)=G_{0}-1,
$$

which in the limit of large gain reduces to Eq. (5.31).

This appearance of finite entropy in a subsystem even when the full system is in a pure state is a purely quantum effect. Classically the entropy of a composite system is at least as large as the entropy of any of its components. Entanglement among the components allows this lower bound on the entropy to be violated in a quantum system. ${ }^{11}$ In this case the two-mode squeezed state has strong entanglement between the signal and idler channels (since their photon numbers are fluctuating identically).

\section{Scattering versus op-amp modes of operation}

We now begin to address the question of how the standard Haus-Caves derivation of the amplifier quantum limit presented in Sec. V.B relates to the general linear-response approach of Sec. IV. Recall that in Sec. III.B.3 we already used this latter approach to discuss position detection with a cavity detector, reaching simi-

\footnotetext{
${ }^{11}$ This paradox has prompted Charles Bennett to remark that a classical house is at least as dirty as its dirtiest room, but a quantum house can be dirty in every room and still perfectly clean over all.
} 


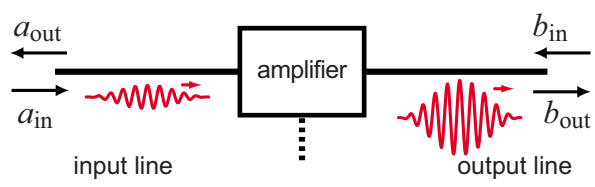

FIG. 9. (Color online) Schematic of a two-port bosonic amplifier. Both the inputs and outputs of the amplifier are attached to transmission lines. The incoming and outgoing wave amplitudes in the input (output) transmission line are labeled $\hat{a}_{\text {in }}, \hat{a}_{\text {out }}\left(\hat{b}_{\text {in }}, \hat{b}_{\text {out }}\right)$, respectively. The voltages at the end of the two lines $\left(\hat{V}_{a}, \hat{V}_{b}\right)$ are linear combinations of incoming and outgoing wave amplitudes.

lar conclusions (i.e., at best, the detector adds noise equal to the zero-point noise). In that linear-responsebased discussion, we saw that a crucial aspect of the quantum limit was the trade-off between back-action noise and measurement-imprecision noise. We saw that reaching the quantum limit required both a detector with ideal noise, as well as an optimization of the detector-oscillator coupling strength. Somewhat disturbingly, none of these ideas appeared explicitly in the Haus-Caves derivation; this can give the misleading impression that the quantum limit never has anything to do with back-action. A further confusion comes from the fact that many detectors have input and outputs that cannot be described by a set of bosonic modes. How does one apply the above arguments to such systems?

The first step in resolving these seeming inconsistencies is to realize that there are really two different ways in which one can use a given amplifier or detector. In deciding how to couple the input signal (i.e., the signal to be amplified) to the amplifier, and in choosing what quantity to measure, the experimentalist essentially enforces boundary conditions; as now shown, there are in general two distinct ways in which to do this. For concreteness, consider the situation shown in Fig. 9: a twoport voltage amplifier where the input and output ports of the amplifier are attached to one-dimensional transmission lines (see Appendix $\mathrm{C}$ for a quick review of quantum transmission lines). As in the previous section, we focus on a narrow bandwidth signal centered about a frequency $\omega$. At this frequency, there exists both a rightmoving and a left-moving wave in each transmission line. We label the corresponding amplitudes in the input (output) line with $a_{\text {in }}, a_{\text {out }}\left(b_{\text {in }}, b_{\text {out }}\right)$, as per Fig. 9. Quantum mechanically these amplitudes become operators, much in the same way that we treated the mode amplitude $a$ as an operator in the previous section. We analyze this two-port bosonic amplifier in Sec. VI; here we only sketch its operation to introduce the two different amplifier operation modes. This will then allow us to understand the subtleties of the Haus-Caves quantum limit derivation.

In the first kind of setup, the experimentalist arranges things so that $a_{\mathrm{in}}$, the amplitude of the wave incident on the amplifier's input port, is precisely equal to the signal to be amplified (i.e., the input signal), irrespective of the amplitude of the wave leaving the input port (i.e., $a_{\text {out }}$ ).
TABLE IV. Two different amplifier modes of operation.

\begin{tabular}{lcc}
\hline \hline Mode & $\begin{array}{c}\text { Input Signal } \\
s(t)\end{array}$ & $\begin{array}{c}\text { Output Signal } \\
o(t)\end{array}$ \\
\hline Scattering & $\begin{array}{c}s(t)=a_{\text {in }}(t) \\
\text { Op-amp }\end{array}$ & $\begin{array}{c}\left.o(t)=b_{\text {out }}(t)\right) \\
\left.\text { indep. of } a_{\text {out }}\right)\end{array}$ \\
$\begin{array}{c}s(t)=V_{a}(t) \\
\left(a_{\text {in }} \text { indep. of } b_{\text {in }}\right) \\
o(t)=V_{b}(t) \\
\left.\text { depends on } a_{\text {out }}\right)\end{array}$ & $\left(b_{\text {out }}\right.$ depends on $\left.b_{\text {in }}\right)$ \\
\hline \hline
\end{tabular}

Further, the output signal is taken to be the amplitude of the outgoing wave exiting the output of the amplifier (i.e., $b_{\text {out }}$ ), again irrespective of whatever might be entering the output port (see Table IV). In this situation, the Haus-Caves description of the quantum limit in the previous section is almost directly applicable; we make this precise in Sec. VI. Back-action is indeed irrelevant, as the prescribed experimental conditions mean that it plays no role. We call this mode of operation the scattering mode, as it is most relevant to time-dependent experiments where the experimentalist launches a signal pulse at the input of the amplifier and looks at what exits the output port. One is usually only interested in the scattering mode of operation in cases where the source producing the input signal is matched to the input of the amplifier: only in this case is the input wave $a_{\text {in }}$ perfectly transmitted into the amplifier. As we see in Sec. VI, such a perfect matching requires a relatively strong coupling between the signal source and the input of the amplifier; as such, the amplifier will strongly enhance the damping of the signal source.

The second mode of linear amplifier operation is what we call the op-amp mode; this is the mode one usually has in mind when thinking of an amplifier which is weakly coupled to the signal source, and will be the next focus. The key difference from the scattering mode is that here the input signal is not simply the amplitude of a wave incident on the input port of the amplifier; similarly, the output signal is not the amplitude of a wave exiting the output port. As such, the Haus-Caves derivation of the quantum limit does not directly apply. For the bosonic amplifier discussed here the op-amp mode would correspond to using the amplifier as a voltage opamp. The input signal would thus be the voltage at the end of the input transmission line. Recall that the voltage at the end of a transmission line involves the amplitude of both left- and right-moving waves, i.e., $V_{a}(t)$ $\propto \operatorname{Re}\left[a_{\text {in }}(t)+a_{\text {out }}(t)\right]$. At first this might seem quite confusing: If the signal source determines $V_{a}(t)$, does this mean it sets the values of both $a_{\text {in }}(t)$ and $a_{\text {out }}(t)$ ? Does not this violate causality? The signal source enforces the value of $V_{a}(t)$ by simply changing $a_{\mathrm{in}}(t)$ in response to the value of $a_{\text {out }}(t)$. While there is no violation of causality, the fact that the signal source is dynamically responding to what comes out of the amplifier's input port implies that back-action is indeed relevant.

The op-amp mode of operation is relevant to the typical situation of weak coupling between the signal source and amplifier input. By weak coupling, we mean here 


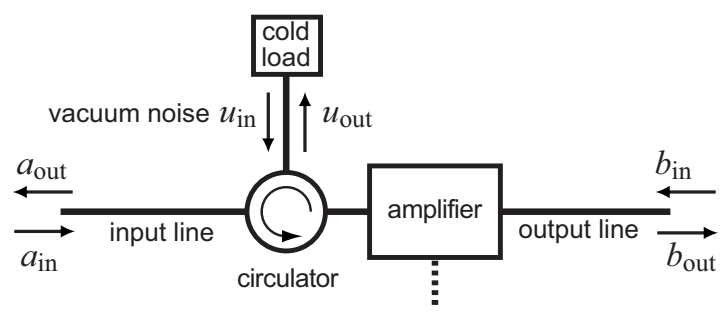

FIG. 10. Illustration of a bosonic two-port amplifier used in the scattering mode of operation. The signal is an incoming wave in the input port of the amplifier, and does not depend on what is coming out of the amplifier. This is achieved by connecting the input line to a circulator and a "cold load" (i.e., a zero-temperature resistor): all that goes back toward the source of the input signal is vacuum noise.

something stronger than just requiring that the amplifier be linear: we require additionally that the amplifier does not appreciably change the dissipation of the signal source. This is analogous to the situation in an ideal voltage op-amp, where the amplifier input impedance is much larger than the impedance of the signal source. We stress that the op-amp mode and this limit of weak coupling is the relevant situation in most electrical measurements.

Thus, we see that the Haus-Caves formulation of the quantum limit is not directly relevant to amplifiers or detectors operated in the usual op-amp mode of operation. We clearly need some other way to describe quantum amplifiers used in this regime. As we demonstrated in the remainder of this section, the general linearresponse approach of Sec. IV is exactly what is needed. To see this, expand the discussion of Sec. IV to include the concepts of input and output impedance as well as power gain. The linear-response approach will allow us to see (similar to Sec. III.B.3) that reaching the quantum limit in the op-amp mode does indeed require a tradeoff between back-action and measurement imprecision, and requires use of an amplifier with ideal quantum noise properties [see Eq. (4.11)]. This approach also has the added benefit of being directly applicable to systems where the input and output of the amplifier are not described by bosonic modes. ${ }^{12}$ In Sec. VI, we return to the scattering description of a two-port voltage amplifier (Fig. 10), and show explicitly how an amplifier can be quantum limited when used in the scattering mode of operation, but miss the quantum limit when used in the op-amp mode of operation.

\section{E. Linear-response description of a position detector}

In this section, we examine the amplifier quantum limit for a two-port linear amplifier in the usual weakcoupling, op-amp regime of operation. Our discussion here will make use of the results obtained for the noise

\footnotetext{
${ }^{12}$ Note that the Haus-Caves derivation for the quantum limit of a scattering amplifier has been generalized to the case of fermionic operators (Gavish et al., 2004).
}

properties of a generic linear-response detector in Sec. IV, including the fundamental quantum noise constraint of Eq. (4.11). For simplicity, we start with the problem of continuous position detection of a harmonic oscillator. Our discussion will thus generalize the discussion of position detection using a cavity detector given in Sec. III.B.3. We start with a generic detector (as introduced in Sec. IV.A) coupled at its input to the position $\hat{x}$ of a harmonic oscillator [see Eq. (4.1)]. ${ }^{13}$ We want to understand the total output noise of our amplifier in the presence of the oscillator, and more importantly how small we can make the amplifier's contribution to this noise. The resulting lower bound is known as the standard quantum limit (SQL) on position detection, and is analogous to the quantum limit on the added noise of a voltage amplifier (discussed in Sec. V.F).

\section{Detector back-action}

We first consider the consequence of noise in the detector input port. As seen in Sec. II.B, the fluctuating back-action force $\hat{F}$ acting on our oscillator will lead to both damping and heating of the oscillator. To model the intrinsic (i.e., detector-independent) heating and damping of the oscillator, we also assume that our oscillator is coupled to an equilibrium heat bath. In the weakcoupling limit that we are interested in, one can use lowest-order perturbation theory in the coupling $A$ to describe the effects of the back-action force $\hat{F}$ on the oscillator. A full quantum treatment (see Appendix I.4) shows that the oscillator is described by an effective classical Langevin equation, ${ }^{14}$

$$
\begin{aligned}
M \ddot{x}(t)= & -M \Omega^{2} x(t)-M \gamma_{0} \dot{x}(t)+F_{0}(t) \\
& -M A^{2} \int d t^{\prime} \gamma\left(t-t^{\prime}\right) \dot{x}\left(t^{\prime}\right)-A F(t) .
\end{aligned}
$$

The position $x(t)$ in the above equation is not an operator, but is simply a classical variable whose fluctuations are driven by the fluctuating forces $F(t)$ and $F_{0}(t)$. Nonetheless, the noise in $x$ calculated from Eq. (5.41) corresponds precisely to $\bar{S}_{x x}[\omega]$, the symmetrized quantummechanical noise in the operator $\hat{x}$. The fluctuating force exerted by the detector (which represents the heating part of the back-action) is described by $A F(t)$ in Eq. (5.41); it has zero mean, and a spectral density given by $A^{2} \bar{S}_{F F}[\omega]$ in Eq. (4.4a). The kernel $\gamma(t)$ describes the damping effect of the detector. It is given by the asym-

\footnotetext{
${ }^{13}$ For consistency with previous sections, our coupling Hamiltonian does not have a minus sign. This is different from the convention of Clerk (2004), where the coupling Hamiltonian is written $H_{\text {int }}=-A \hat{x} \cdot \hat{F}$.

${ }^{14}$ Note that we have omitted a back-action term in this equation which leads to small renormalizations of the oscillator frequency and mass. These terms are not important for the following discussion, so we have omitted them for clarity; one can consider $M$ and $\Omega$ in this equation to be renormalized quantities. See Appendix I.4 for more details.
} 
metric part of the detector's quantum noise, as was derived in Sec. II.B [see Eq. (2.12)].

Equation (5.41) also describes the effects of an equilibrium heat bath at temperature $T_{0}$ which models the intrinsic (i.e., detector-independent) damping and heating of the oscillator. The parameter $\gamma_{0}$ is the damping arising from this bath and $F_{0}$ is the corresponding fluctuating force. The spectral density of the $F_{0}$ noise is determined by $\gamma_{0}$ and $T_{0}$ via the fluctuation-dissipation theorem [see Eq. (2.16)]. $T_{0}$ and $\gamma_{0}$ have a simple physical significance: they are the temperature and damping of the oscillator when the coupling to the detector $A$ is set to zero.

To make further progress, we recall from Sec. II.B that even though our detector will in general not be in equilibrium, we may nonetheless assign it an effective temperature $T_{\text {eff }}[\omega]$ at each frequency [see Eq. (2.8)]. The effective temperature of an out-of-equilibrium detector is simply a measure of the asymmetry of the detector's quantum noise. We are often interested in the limit where the internal detector time scales are much faster than the time scales relevant to the oscillator (i.e., $\left.\Omega^{-1}, \gamma^{-1}, \gamma_{0}^{-1}\right)$. We may then take the $\omega \rightarrow 0$ limit in the expression for $T_{\text {eff }}$, yielding

$$
2 k_{B} T_{\text {eff }} \equiv \bar{S}_{F F}(0) / M \gamma(0) .
$$

In this limit, the oscillator position noise calculated from Eq. (5.41) is given by

$$
\bar{S}_{x x}[\omega]=\frac{1}{M} \frac{2\left(\gamma_{0}+\gamma\right) k_{B}}{\left(\omega^{2}-\Omega^{2}\right)^{2}+\omega^{2}\left(\gamma+\gamma_{0}\right)^{2}} \frac{\gamma_{0} T+\gamma T_{\mathrm{eff}}}{\gamma_{0}+\gamma} .
$$

This is exactly what would be expected if the oscillator were only attached to an equilibrium Ohmic bath with a damping coefficient $\gamma_{\Sigma}=\gamma_{0}+\gamma$ and temperature $\bar{T}$ $=\left(\gamma_{0} T+\gamma T_{\text {eff }}\right) / \gamma_{\Sigma}$.

\section{Total output noise}

The next step in our analysis is to link fluctuations in the position of the oscillator [as determined from Eq. (5.41)] to noise in the output of the detector. As discussed in Sec. III.B.3, the output noise consists of the intrinsic output noise of the detector (i.e., "measurement-imprecision noise") plus the amplified position fluctuations in the position of the oscillator. The latter contains both an intrinsic part and a term due to the response of the oscillator to the back-action.

To start, imagine that we can treat both the oscillator position $x(t)$ and the detector output $I(t)$ as classically fluctuating quantities. Using the linearity of the detector's response, we can then write $\delta I_{\text {total }}$, the fluctuating part of the detector's output, as

$$
\delta I_{\text {total }}[\omega]=\delta I_{0}[\omega]+A \chi_{I F}[\omega] \delta x[\omega] .
$$

The first term $\left(\delta I_{0}\right)$ describes the intrinsic (oscillatorindependent) fluctuations in the detector output, and has a spectral density $\bar{S}_{I I}[\omega]$. If we scale this by $\left|\chi_{I F}\right|^{2}$, we have the measurement-imprecision noise discussed in Sec. III.B.3. The second term corresponds to the amplified fluctuations of the oscillator, which are in turn given by solving Eq. (5.41),

$$
\begin{aligned}
\delta x[\omega] & =-\left[\frac{1 / M}{\left(\omega^{2}-\Omega^{2}\right)+i \omega \Omega / Q[\omega]}\right]\left(F_{0}[\omega]-A F[\omega]\right) \\
& \equiv \chi_{x x}[\omega]\left(F_{0}[\omega]-A F[\omega]\right),
\end{aligned}
$$

where $Q[\omega]=\Omega /\left(\gamma_{0}+\gamma[\omega]\right)$ is the oscillator quality factor. It follows that the spectral density of the total noise in the detector output is given classically by

$$
\begin{aligned}
\mathcal{S}_{I I, \text { tot }}[\omega]= & \mathcal{S}_{I I}[\omega]+\left|\chi_{x x}[\omega] \chi_{I F}[\omega]\right|^{2}\left(A^{4} \mathcal{S}_{F F}[\omega]\right. \\
& \left.+A^{2} \mathcal{S}_{F_{0} F_{0}}[\omega]\right) \\
& +2 A^{2} \operatorname{Re}\left[\chi_{x x}[\omega] \chi_{I F}[\omega] \mathcal{S}_{I F}[\omega]\right] .
\end{aligned}
$$

Here $\mathcal{S}_{I I}, \mathcal{S}_{F F}$, and $\mathcal{S}_{I F}$ are the (classical) detector noise correlators calculated in the absence of any coupling to the oscillator. Note importantly that we have included the fact that the two kinds of detector noise (in $\hat{I}$ and in $\hat{F})$ may be correlated with one another.

To apply the classically derived Eq. (5.46) to our quantum detector-plus-oscillator system, we recall from Sec. II.B that symmetrized quantum noise spectral densities play the role of classical noise. The LHS of Eq. (5.46) thus becomes $S_{I I, \text { tot}}$, the total symmetrized quantummechanical output noise of the detector, while the RHS will now contain the symmetrized quantum-mechanical detector noise correlators $\bar{S}_{F F}, \bar{S}_{I I}$, and $\bar{S}_{I F}$, defined as in Eq. (4.4a). Though this may seem rather ad hoc, one can easily demonstrate that Eq. (5.46) thus interpreted would be quantum mechanically rigorous if the detector correlation functions obeyed Wick's theorem. Thus, quantum corrections to Eq. (5.46) will arise solely from the non-Gaussian nature of the detector noise correlators. We expect from the central limit, theorem that such corrections will be small in the relevant limit, where $\omega$ is much smaller that the typical detector frequency $\sim k_{B} T_{\text {eff }} / \hbar$, and neglect these corrections in what follows. Note that the validity of Eq. (5.46) for a specific model of a tunnel junction position detector has been explicitly verified by Clerk and Girvin (2004).

\section{Detector power gain}

Before proceeding, we need to consider our detector once again in isolation, and return to the fundamental question of what we mean by amplification. To be able to say that our detector truly amplifies the motion of the oscillator, it is not sufficient to simply say that the response function $\chi_{I F}$ must be large (note that $\chi_{I F}$ is not dimensionless). Instead, true amplification requires that the power delivered by the detector to a following amplifier be much larger than the power drawn by the detector at its input-i.e., the detector must have a dimensionless power gain $G_{P}[\omega]$ much larger than 1. As discussed, if the power gain was not large, we would need to worry about the next stage in the amplification 


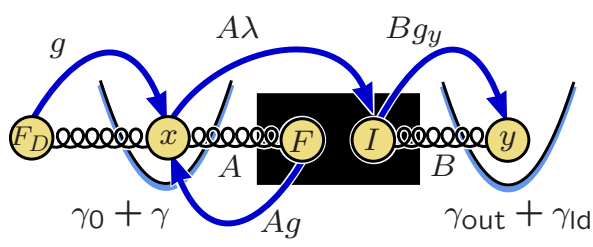

FIG. 11. (Color online) Schematic of a generic linear-response position detector, where an auxiliary oscillator $y$ is driven by the detector output.

of our signal, and how much noise is added in that process. Having a large power gain means that by the time our signal reaches the following amplifier, it is so large that the added noise of this following amplifier is unimportant. The power gain is analogous to the dimensionless photon-number gain $G$ that appears in the standard Haus-Caves description of a bosonic linear amplifier [see Eq. (5.7)].

To make the above more precise, we start with the idea case of no reverse gain $\chi_{F I}=0$. We define the power gain $G_{P}[\omega]$ of our generic position detector in a way that is analogous to the power gain of a voltage amplifier. Imagine we drive the oscillator we are trying to measure with a force $2 F_{D} \cos \omega t$; this will cause the output of our detector $\langle\hat{I}(t)\rangle$ to also oscillate at frequency $\omega$. To optimally detect this signal in the detector output, we further couple the detector output $I$ to a second oscillator with natural frequency $\omega$, mass $M$, and position $y$ : there is a new coupling term in our Hamiltonian $H_{\mathrm{int}}^{\prime}=B \hat{I} \cdot \hat{y}$, where $B$ is a coupling strength. The oscillations in $\langle I(t)\rangle$ will now act as a driving force on the auxiliary oscillator $y$ (see Fig 11). We can consider the auxiliary oscillator $y$ as a "load" we are trying to drive with the output of our detector.

To find the power gain, we need to consider both $P_{\text {out }}$, the power supplied to the output oscillator $y$ from the detector, and $P_{\text {in }}$, the power fed into the input of the amplifier. Consider first $P_{\text {in }}$. This is simply the timeaveraged power dissipation of the input oscillator $x$ caused by the back-action damping $\gamma[\omega]$. Using an overbar to denote a time average, we have

$$
P_{\text {in }} \equiv M \gamma[\omega] \overline{\dot{x}^{2}}=M \gamma[\omega] \omega^{2}\left|\chi_{x x}[\omega]\right|^{2} F_{D}^{2} .
$$

Note that the oscillator susceptibility $\chi_{x x}[\omega]$ depends on both the back-action damping $\gamma[\omega]$ and the intrinsic oscillator damping $\gamma_{0}$ [see Eq. (5.45)].

Next, we need to consider the power supplied to the load oscillator $y$ at the detector output. This oscillator will have some intrinsic detector-independent damping $\gamma_{\mathrm{ld}}$, as well as a back-action damping $\gamma_{\text {out }}$. In the same way that the back-action damping $\gamma$ of the input oscillator $x$ is determined by the quantum noise in $\hat{F}$ [see Eqs. (2.14), (2.13), and (2.12)], the back-action damping of the load oscillator $y$ is determined by the quantum noise in the output operator $\hat{I}$,

$$
\begin{aligned}
\gamma_{\text {out }}[\omega] & =\left(B^{2} / M \omega\right)\left[-\operatorname{Im} \chi_{I I}[\omega]\right] \\
& =\left(B^{2} / M \hbar \omega\right)\left[\frac{S_{I I}[\omega]-S_{I I}[-\omega]}{2}\right],
\end{aligned}
$$

where $\chi_{I I}$ is the linear-response susceptibility which determines how $\langle\hat{I}\rangle$ responds to a perturbation coupling to $\hat{I}$

$$
\chi_{I I}[\omega]=-\frac{i}{\hbar} \int_{0}^{\infty} d t\langle[\hat{I}(t), \hat{I}(0)]\rangle e^{i \omega t} .
$$

As the oscillator $y$ is being driven on resonance, the relation between $y$ and $I$ is given by $y[\omega]=\chi_{y y}[\omega] I[\omega]$ with $\chi_{y y}[\omega]=-i\left[\omega M \gamma_{\text {out }}[\omega]\right]^{-1}$. From conservation of energy, we have that the net power flow into the output oscillator from the detector is equal to the power dissipated out of the oscillator through the intrinsic damping $\gamma_{\mathrm{ld}}$. We thus have

$$
\begin{aligned}
P_{\text {out }} & \equiv M \gamma_{\mathrm{ld}} \overline{\dot{y}^{2}} \\
& =M \gamma_{\mathrm{ld}} \omega^{2}\left|\chi_{y y}[\omega]\right|^{2}\left|B A \chi_{I F} \chi_{x x}[\omega] F_{D}\right|^{2} \\
& =\frac{1}{M} \frac{\gamma_{\mathrm{ld}}}{\left(\gamma_{\mathrm{ld}}+\gamma_{\text {out }}[\omega]\right)^{2}}\left|B A \chi_{I F} \chi_{x x}[\omega] F_{D}\right|^{2} .
\end{aligned}
$$

Using the above definitions, we find that the ratio between $P_{\text {out }}$ and $P_{\text {in }}$ is independent of $\gamma_{0}$, but depends on $\gamma_{\text {ld }}$

$$
\frac{P_{\text {out }}}{P_{\text {in }}}=\frac{1}{M^{2} \omega^{2}} \frac{A^{2} B^{2}\left|\chi_{I F}[\omega]\right|^{2}}{\gamma_{\text {out }}[\omega] \gamma[\omega]} \frac{\gamma_{\text {ld }} / \gamma_{\text {out }}[\omega]}{\left(1+\gamma_{\text {ld }} / \gamma_{\text {out }}[\omega]\right)^{2}} .
$$

We now define the detector power gain $G_{P}[\omega]$ as the value of this ratio maximized over the choice of $\gamma_{\mathrm{ld}}$. The maximum occurs for $\gamma_{\mathrm{ld}}=\gamma_{\mathrm{out}}[\omega]$ (i.e., the load oscillator is "matched" to the output of the detector), resulting in

$$
\begin{aligned}
G_{P}[\omega] \equiv \max \left[\frac{P_{\text {out }}}{P_{\text {in }}}\right] & =\frac{1}{4 M^{2} \omega^{2}} \frac{A^{2} B^{2}\left|\chi_{I F}\right|^{2}}{\gamma_{\text {out }} \gamma} \\
& =\frac{\left|\chi_{I F}[\omega]\right|^{2}}{4 \operatorname{Im} \chi_{F F}[\omega] \operatorname{Im} \chi_{I I}[\omega]} .
\end{aligned}
$$

In the last equality, we have used the relation between the damping rates $\gamma[\omega]$ and $\gamma_{\text {out }}[\omega]$ and the linearresponse susceptibilities $\chi_{F F}[\omega]$ and $\chi_{I I}[\omega]$ [see Eqs. (2.15) and (5.48)]. We thus find that the power gain is a simple dimensionless ratio formed by the three different response coefficients characterizing the detector, and is independent of the coupling constants $A$ and $B$. As we see in Sec. V.F, it is completely analogous to the power gain of a voltage amplifier, which is also determined by three parameters: the voltage gain, the input impedance, and the output impedance. Note that there are other important measures of power gain commonly in use in the engineering community: we comment on these in Sec. VII.B.

Finally, the above results are easily generalized to the case where the detector's reverse gain $\chi_{F I}$ is nonvanishing. For simplicity, we present results for the case where 
$\beta=\operatorname{Re}\left(\chi_{I F} \chi_{F I}\right) /\left|\chi_{I F}\right|^{2} \geqslant 0$, implying that there is no positive feedback. Maximizing the ratio of $P_{\text {out }} / P_{\text {in }}$ over choices of $\gamma_{l d}$ now yields

$$
G_{P, \mathrm{rev}}=2 G_{P} /\left(1+2 \beta G_{P}+\sqrt{1+4 \beta G_{P}}\right) \leqslant 1 / \beta .
$$

Here $G_{P \text {,rev }}$ is the power gain in the presence of reverse gain, while $G_{P}$ is the zero-reverse gain power gain given by Eq. (5.52). One can confirm that $G_{P, \text { rev }}$ is a monotonic increasing function of $G_{P}$, and is bounded by $1 / \beta$. As noted in Sec. IV.A.4, if $\chi_{F I}=\chi_{I F}^{*}$, there is no additional quantum noise constraint on our detector beyond what exists classically [i.e., the RHS of Eq. (4.11) vanishes]. We now see explicitly that when $\chi_{F I}=\chi_{I F}^{*}$, the power gain of our detector can be at most 1 , as $\beta=1$. Thus, while there is no minimum back-action noise required by quantum mechanics in this case, there is also no amplification: at best, our detector would act as a transducer. Note further that if the detector has $\chi_{F I}=\chi_{I F}^{*}$ and optimizes the inequality of Eq. (4.11), then one can show $G_{P, \text { rev }}$ must be 1 (see Appendix I.2): the detector is simply a transducer. This is in keeping with the results obtained using the Haus-Caves approach, which also yields the conclusion that a noiseless detector is a transducer.

\section{Simplifications for a quantum-ideal detector}

We now consider the important case where our detector has no reverse gain (allowing it to have a large power gain), and also has ideal quantum noise [i.e., it satisfies the ideal noise condition of Eq. (4.17)]. Fulfilling this condition immediately places some powerful constraints on our detector.

First, note that we have defined in Eq. (5.42) the effective temperature of our detector based on what happens at the input port; this is the effective temperature seen by the oscillator we are trying to measure. We could also consider the effective temperature of the detector as seen at the output (i.e., by the oscillator $y$ used in defining the power gain). This output effective temperature is determined by the quantum noise in the output operator $\hat{I}$,

$$
k_{B} T_{\text {eff,out }}[\omega] \equiv \hbar \omega / \log \left(S_{I I}[+\omega] / S_{I I}[-\omega]\right) .
$$

For a general out-of-equilibrium amplifier, $T_{\text {eff,out }}$ does not have to be equal to the input effective temperature $T_{\text {eff }}$ defined by Eq. (2.8). However, for a quantum-ideal detector, the effective proportionality between input and output operators [see Eq. (I13)] immediately yields

$$
T_{\text {eff,out }}[\omega]=T_{\text {eff }}[\omega] \text {. }
$$

Thus, a detector with quantum-ideal noise necessarily has the same effective temperature at its input and its output. This is all the more remarkable given that a quantum-ideal detector cannot be in equilibrium, and thus $T_{\text {eff }}$ cannot represent a real physical temperature.

Another important simplification for a quantum-ideal detector is the expression for the power gain. Using the proportionality between input and output operators [cf. Eq. (I13)], one finds

$$
G_{P}[\omega]=\frac{(\operatorname{Im} \alpha)^{2} \operatorname{coth}^{2}\left(\hbar \omega / 2 k_{B} T_{\mathrm{eff}}\right)+(\operatorname{Re} \alpha)^{2}}{|\alpha|^{2}},
$$

where $\alpha[\omega]$ is the parameter characterizing a quantumlimited detector in Eq. (4.18); recall that $|\alpha[\omega]|^{2}$ determines the ratio of $S_{I I}$ and $S_{F F}$. It follows immediately that for a detector with ideal noise to also have a large power gain $\left(G_{P} \gg 1\right)$, one absolutely needs $k_{B} T_{\text {eff }} \gg \hbar \omega$ : a large power gain implies a large effective detector temperature. In the large- $G_{P}$ limit, we have

$$
G_{P} \simeq\left[\frac{\operatorname{Im} \alpha}{|\alpha|} \frac{k_{B} T_{\text {eff }}}{\hbar \omega / 2}\right]^{2} .
$$

Thus, the effective temperature of a quantum-ideal detector does more than just characterize the detector back-action-it also determines the power gain.

Finally, an additional consequence of the large- $G_{P}[\omega]$, large $T_{\text {eff }}$ limit is that the gain $\chi_{I F}$ and noise cross correlator $\bar{S}_{I F}$ are in phase: $\bar{S}_{I F} / \chi_{I F}$ is purely real, up to corrections that are as small as $\omega / T_{\text {eff. This is shown }}$ explicitly in Appendix I.3. Thus, we find that a large power gain detector with ideal quantum noise cannot have significant out-of-phase correlations between its output and input noises. This last point may be understood in terms of the idea of wasted information: if there were significant out-of-phase correlations between $\hat{I}$ and $\hat{F}$, it would be possible to improve the performance of the amplifier by using feedback. We discuss this point in Sec. VI. Note that because $\bar{S}_{I F} / \chi_{I F}$ is real the last term in the quantum noise constraint of Eq. (4.11) vanishes.

\section{Quantum limit on added noise and noise temperature}

We now turn to calculating the noise added to our signal [i.e., $\langle\hat{x}(t)\rangle]$ by our generic position detector. To characterize this added noise, it is useful to take the total (symmetrized) noise in the output of the detector and refer it back to the input by dividing out the gain of the detector,

$$
\bar{S}_{x x, \text { tot }}[\omega] \equiv \bar{S}_{I I, \text { tot }}[\omega] / A^{2}\left|\chi_{I F}[\omega]\right|^{2} .
$$

$\bar{S}_{x x, \text { tot }}[\omega]$ is simply the frequency-dependent spectral density of position fluctuations inferred from the output of the detector. It is this quantity that will directly determine the sensitivity of the detector-given a certain detection bandwidth, what is the smallest variation of $x$ that can be resolved? The quantity $\bar{S}_{x x, \text { tot }}[\omega]$ will have contributions from the intrinsic fluctuations of the input signal as well as a contribution due to the detector. We first define $\bar{S}_{x x \text {, eq }}[\omega, T]$ to be the symmetrized equilibrium position noise of our damped oscillator (whose damping is $\gamma_{0}+\gamma$ ) at temperature $T$, 


$$
\bar{S}_{x x, \mathrm{eq}}[\omega, T]=\hbar \operatorname{coth}\left(\hbar \omega / 2 k_{B} T\right)\left(-\operatorname{Im} \chi_{x x}[\omega]\right),
$$

where the oscillator susceptibility $\chi_{x x}[\omega]$ is defined in Eq. (5.45). The total inferred position noise may then be written as

$$
\bar{S}_{x x, \text { tot }}[\omega] \equiv\left[\gamma_{0} /\left(\gamma_{0}+\gamma\right)\right] \cdot \bar{S}_{x x, \mathrm{eq}}\left[\omega, T_{0}\right]+\bar{S}_{x x, \text { add }}[\omega] .
$$

In the usual case where the detector noise can be approximated as being white, this spectral density will consist of a Lorentzian sitting atop a constant noise floor (see Fig. 6). The first term in Eq. (5.60) represents position noise arising from the fluctuating force $\delta F_{0}(t)$ associated with the intrinsic (detector-independent) dissipation of the oscillator [see Eq. (5.41)]. The prefactor of this term arises because the strength of the intrinsic Langevin force acting on the oscillator is proportional to $\gamma_{0}$, not to $\gamma_{0}+\gamma$.

The second term in Eq. (5.60) represents the added position noise due to the detector. It has contributions both from the detector's intrinsic output noise $\bar{S}_{I I}$ from the detector's back-action noise $\bar{S}_{F F}$, and may be written as

$$
\begin{aligned}
\bar{S}_{x x, \text { add }}[\omega]= & \frac{\bar{S}_{I I}}{\left|\chi_{I F}\right|^{2} A^{2}}+A^{2}\left|\chi_{x x}\right|^{2} \bar{S}_{F F} \\
& +\frac{2 \operatorname{Re}\left[\chi_{I F}^{*}\left(\chi_{x x}\right)^{*} \bar{S}_{I F}\right]}{\left|\chi_{I F}\right|^{2}} .
\end{aligned}
$$

For clarity, we have omitted writing the explicit frequency dependence of the gain $\chi_{I F}$, susceptibility $\chi_{x x}$, and noise correlators; they should all be evaluated at the frequency $\omega$. Note that the first term on the RHS corresponds to the measurement-imprecision noise of our detector, $\bar{S}_{x x}^{I}(\omega)$.

We can now finally address the quantum limit on the added noise in this setup. As discussed in Sec. V.D, the Haus-Caves derivation of the quantum limit (cf. Sec. V.B) is not directly applicable to the position detector we are describing here; nonetheless, we may use its result to guess what form the quantum limit will take here. The Haus-Caves argument told us that the added noise of a phase-preserving linear amplifier must be at least as large as the zero-point noise. We thus anticipate that, if our detector has a large power gain, the spectral density of the noise added by the detector (i.e., $\bar{S}_{x x \text {,add }}[\omega]$ ) must be at least as large as the zero-point noise of our damped oscillator,

$$
\bar{S}_{x x, \text { add }}[\omega] \geqslant \lim _{T \rightarrow 0} \bar{S}_{x x, \mathrm{eq}}[w, T]=\left|\hbar \operatorname{Im} \chi_{x x}[\omega]\right| .
$$

We now show that the bound above is rigorously correct at each frequency $\omega$.

The first step is to examine the dependence of the added noise $\bar{S}_{x x \text {,add }}[\omega]$ [as given by Eq. (5.61)] on the coupling strength $A$. If we ignore for a moment the detector-dependent damping of the oscillator, the situa- tion is the same as the cavity position detector of Sec. III.B.3: there is an optimal value of the coupling strength $A$ which corresponds to a trade-off between imprecision noise and back-action [i.e., the first and second terms in Eq. (5.61)]. We thus expect $\bar{S}_{x x \text {,add }}[\omega]$ to attain a minimum value at an optimal choice of coupling $A$ $=A_{\text {opt }}$ where both these terms make equal contributions (see Fig. 5). Defining $\phi[\omega]=\arg \chi_{x x}[\omega]$, we thus have the bound

$$
\begin{aligned}
& \bar{S}_{x x, \text { add }}[\omega] \geqslant 2\left|\chi_{x x}[\omega]\right|\left[\sqrt{\frac{\bar{S}_{I I} \bar{S}_{F F}}{\left|\chi_{I F}\right|^{2}}}\right. \\
&\left.+\frac{\operatorname{Re}\left[\chi_{I F}^{*} e^{-i \phi[\omega]} \bar{S}_{I F}\right]}{\left|\chi_{I F}\right|^{2}}\right],
\end{aligned}
$$

where the minimum value at frequency $\omega$ is achieved when

$$
A_{\mathrm{opt}}^{2}=\sqrt{\bar{S}_{I I}[\omega] /\left|\chi_{I F}[\omega] \chi_{x x}[\omega]\right|^{2} \bar{S}_{F F}[\omega]}
$$

Using the inequality $X^{2}+Y^{2} \geqslant 2|X Y|$ we see that this value serves as a lower bound on $\bar{S}_{x x \text {,add }}$ even in the presence of detector-dependent damping. In the case where the detector-dependent damping is negligible, the RHS of Eq. (5.63) is independent of $A$, and thus Eq. (5.64) can be satisfied by simply tuning the coupling strength $A$; in the more general case where there is detector-dependent damping, the RHS is also a function of $A$ (through the response function $\chi_{x x}[\omega]$ ), and it may no longer be possible to achieve Eq. (5.64) by simply tuning $A{ }^{15}$

While Eq. (5.63) is certainly a bound on the added displacement noise $\bar{S}_{x x \text {,add }}[\omega]$, it does not in itself represent the quantum limit. Reaching the quantum limit requires more than simply balancing the detector backaction and intrinsic output noises [i.e., the first two terms in Eq. (5.61)]; one also needs a detector with quantumideal noise properties, that is a detector which satisfies Eq. (4.17). Using the quantum noise constraint of Eq. (4.11) to further bound $\bar{S}_{x x \text {,add }}[\omega]$, we obtain

$$
\begin{aligned}
\bar{S}_{x x, \mathrm{add}}[\omega] \geqslant & 2\left|\frac{\chi_{x x}[\omega]}{\chi_{I F}}\right| \\
& \times\left[\sqrt{\left(\frac{\hbar\left|\chi_{I F}\right|}{2}\right)^{2}\left(1+\Delta\left[\frac{2 \bar{S}_{I F}}{\hbar \chi_{I F}}\right]\right)+\left|\bar{S}_{I F}\right|^{2}}\right. \\
& \left.+\frac{\operatorname{Re}\left[\chi_{I F}^{*} e^{-i \phi[\omega]} \bar{S}_{I F}\right]}{\left|\chi_{I F}\right|}\right],
\end{aligned}
$$

where the function $\Delta[z]$ is defined in Eq. (4.12). The minimum value of $\bar{S}_{x x \text {,add }}[\omega]$ in Eq. (5.65) is now achieved when one has both an optimal coupling [i.e.,

\footnotetext{
${ }^{15}$ Note that, in the heuristic discussion of position detection using a resonant cavity detector in Sec. III.B.3, these concerns did not arise as there was no back-action damping.
} 
Eq. (5.64)] and a quantum-limited detector, that is one which satisfies Eq. (4.11) as an equality.

Next, we consider the relevant case where our detector is a good amplifier and has a power gain $G_{P}[\omega] \gg 1$ over the width of the oscillator resonance. As discussed, this implies that the ratio $\bar{S}_{I F} / \chi_{I F}$ is purely real, up to small $\hbar \omega / k_{B} T_{\text {eff }}$ corrections (see Sec. IV.A.4 and Appendix I.3 for more details). This in turn implies that $\Delta\left[2 \bar{S}_{I F} / \hbar \chi_{I F}\right]=0$; we thus have

$$
\begin{aligned}
\bar{S}_{x x, \text { add }}[\omega] \geqslant & 2\left|\chi_{x x}[\omega]\right|\left[\sqrt{\left(\frac{\hbar}{2}\right)^{2}+\left(\frac{\bar{S}_{I F}}{\chi_{I F}}\right)^{2}}\right. \\
& \left.+\frac{\cos [\phi[\omega]] \bar{S}_{I F}}{\chi_{I F}}\right] .
\end{aligned}
$$

Finally, as there is no further constraint on $\bar{S}_{I F} / \chi_{I F}$ (beyond the fact that it is real), we can minimize the expression over its value. The minimum $\bar{S}_{x x \text {,add }}[\omega]$ is achieved for a detector whose cross correlator satisfies

$$
\bar{S}_{I F}[\omega] /\left.\chi_{I F}\right|_{\text {optimal }}=-(\hbar / 2) \cot \phi[\omega],
$$

with the minimum value given by

$$
\left.\bar{S}_{x x, \operatorname{ad}\lceil}[\omega]\right|_{\min }=\hbar\left|\operatorname{Im} \chi_{x x}[\omega]\right|=\lim _{T \rightarrow 0} \bar{S}_{x x, \mathrm{eq}}[\omega, T],
$$

where $\bar{S}_{x x, \text { eq }}[\omega, T]$ is the equilibrium contribution to $\bar{S}_{x x, \text { tot }}[\omega]$ defined in Eq. (5.59). Thus, in the limit of a large power gain, we have that at each frequency the minimum displacement noise added by the detector is precisely equal to the noise arising from a zerotemperature bath. This conclusion is irrespective of the strength of the intrinsic (detector-independent) oscillator damping.

We have thus derived the amplifier quantum limit (in the context of position detection) for a two-port amplifier used in the op-amp mode of operation. Though we reached a conclusion similar to that given by the HausCaves approach, the linear-response, quantum noise approach used is quite different. This approach makes explicitly clear what is needed to reach the quantum limit. We find that to reach the quantum limit on the added displacement noise $\bar{S}_{x x \text {,add }}[\omega]$ with a large power gain, one needs (1) a quantum-limited detector, that is, a detector which satisfies the ideal noise condition of Eq. (4.17), and hence the proportionality condition of Eq. (I13); (2) a coupling $A$ which satisfies Eq. (5.64); and (3) a detector cross correlator $\bar{S}_{I F}$ that satisfies Eq. (5.67).

Recall that condition (1) is identical to what is required for quantum-limited detection of a qubit; it is rather demanding, and requires that there is no wasted information about the input signal in the detector which is not revealed in the output (Clerk et al., 2003). Also note that $\cot \phi$ changes quickly as a function of frequency across the oscillator resonance, whereas $\bar{S}_{I F}$ will be roughly constant; condition (2) thus implies that it will not be possible to achieve a minimal $\bar{S}_{x x, \text { add }}[\omega]$ across the entire oscillator resonance. A more reasonable goal is to optimize $\bar{S}_{x x, \text { add }}[\omega]$ at resonance, $\omega=\Omega$. As $\chi_{x x}[\Omega]$ is imaginary, Eq. (5.67) tells us that $\bar{S}_{I F}$ should be zero. Assuming we have a quantum-limited detector with a large power gain $\left(k_{B} T_{\text {eff }} \gg \hbar \Omega\right)$, the remaining condition on the coupling $A$ [Eq. (5.64)] may be written as

$$
\frac{\gamma\left[A_{\mathrm{opt}}\right]}{\gamma_{0}+\gamma\left[A_{\mathrm{opt}}\right]}=\left|\frac{\operatorname{Im} \alpha}{\alpha}\right| \frac{1}{2 \sqrt{G_{P}[\Omega]}}=\frac{\hbar \Omega}{4 k_{B} T_{\mathrm{eff}}} .
$$

As $\gamma[A] \propto A^{2}$ is the detector-dependent damping of the oscillator, we thus have that to achieve the quantumlimited value of $\bar{S}_{x x, \text { add }}[\Omega]$ with a large power gain, one needs the intrinsic damping of the oscillator to be much larger than the detector-dependent damping. The detector-dependent damping must be small enough to compensate the large effective temperature of the detector; if the bath temperature satisfies $\hbar \Omega / k_{B} \ll T_{\text {bath }}$ $\ll T_{\text {eff }}$, Eq. (5.69) implies that at the quantum limit the temperature of the oscillator will be given by

$$
T_{\text {osc }} \equiv\left(\gamma \cdot T_{\text {eff }}+\gamma_{0} \cdot T_{\text {bath }}\right) /\left(\gamma+\gamma_{0}\right) \rightarrow \hbar \Omega / 4 k_{B}+T_{\text {bath }} .
$$

Thus, at the quantum limit and for large $T_{\text {eff }}$, the detector raises the oscillator's temperature by $\hbar \Omega / 4 k_{B}{ }^{16}$ As expected, this additional heating is only half the zeropoint energy; in contrast, the quantum-limited value of $\bar{S}_{x x, \text { add }}[\omega]$ corresponds to the full zero-point result, as it also includes the contribution of the intrinsic output noise of the detector.

Finally, we return to Eq. (5.65); this is the constraint on the added noise $\bar{S}_{x x \text {,add }}[\omega]$ before we assumed our detector to have a large power gain, and consequently a large $T_{\text {eff }}$. Note crucially that if we did not require a large power gain, then there need not be any added noise. Without the assumption of a large power gain, the ratio $\bar{S}_{I F} / \chi_{I F}$ can be made imaginary with a large magnitude. In this limit, $1+\Delta\left[2 \bar{S}_{I F} / \chi_{I F}\right] \rightarrow 0$ : the quantum constraint on the amplifier noises [e.g., the RHS of Eq. (4.11)] vanishes. One can then easily use Eq. (5.65) to show that the added noise $\bar{S}_{x x \text {,add }}[\omega]$ can be zero. This confirms a general conclusion that we have seen several times now (see Secs. IV.A.4 and V.B): if a detector does not amplify (i.e., the power gain is unity), it need not produce any added noise.

\section{F. Quantum limit on the noise temperature of a voltage amplifier}

We now turn our attention to the quantum limit on the added noise of a generic linear voltage amplifier

\footnotetext{
${ }^{16}$ If in contrast our oscillator was initially at zero temperature (i.e., $T_{\text {bath }}=0$ ), one finds that the effect of the back-action (at the quantum limit and for $G_{P} \gg 1$ ) is to heat the oscillator to a temperature $\hbar \Omega / k_{B} \ln 5$.
} 


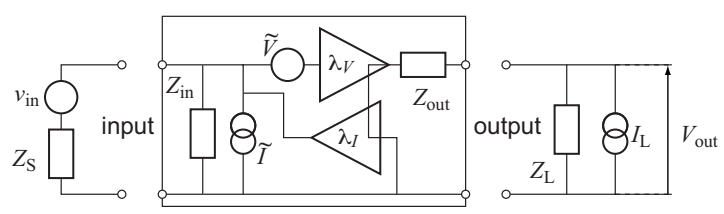

FIG. 12. Schematic of a linear voltage amplifier, including a reverse gain $\lambda_{I}$. $\tilde{V}$ and $\tilde{I}$ represent the standard voltage and current noises of the amplifier, as discussed in the text. The case with reverse gain is discussed in Sec. VI.

used in the op-amp mode of operation [see, e.g., Devoret and Schoelkopf (2000)]. For such amplifiers, the added noise is usually expressed in terms of the "noise temperature" of the amplifier; we define this concept and demonstrate that, when appropriately defined, this noise temperature must be bigger than $\hbar \omega /\left(2 k_{B}\right)$, where $\omega$ is the signal frequency. Though the voltage amplifier is closely analogous to the position detector treated previously, its importance makes it worthy of a separate discussion. As in the previous section, our discussion here will use the general linear-response approach. In contrast, in Sec. VI, we present the bosonic scattering description of a two-port voltage amplifier, a description similar to that used in formulating the Haus-Caves proof of the amplifier quantum limit. We will then be in a good position to contrast the linear-response and scattering approaches and will see there that the scattering and the op-amp modes of operation discussed here are not equivalent. We stress that the general treatment presented here can also be applied directly to the system discussed in Sec. VI.

\section{Classical description of a voltage amplifier}

We begin by recalling the standard schematic description of a voltage amplifier (see Fig. 12). The input voltage to be amplified $v_{\text {in }}(t)$ is produced by a circuit which has a Thevenin-equivalent impedance $Z_{s}$, the source impedance. We stress that we are considering the op-amp mode of amplifier operation, and thus the input signal does not correspond to the amplitude of a wave incident upon the amplifier (see Sec. V.D). The amplifier itself has an input impedance $Z_{\text {in }}$ and an output impedance $Z_{\text {out }}$, as well as a voltage gain coefficient $\lambda_{V}$ : assuming no current is drawn at the output (i.e., $Z_{\text {load }} \rightarrow \infty$ in Fig. 12), the output voltage $V_{\text {out }}(t)$ is simply $\lambda_{V}$ times the voltage across the input terminals of the amplifier.

The added noise of the amplifier is usually represented by two noise sources placed at the amplifier in-

put. There is both a voltage noise source $\tilde{V}(t)$ in series with the input voltage source and a current noise source $\tilde{I}(t)$ in parallel with the input voltage source (Fig. 12). The voltage noise produces a fluctuating voltage $\tilde{V}(t)$ (spectral density $\mathcal{S}_{\tilde{V} \tilde{V}}[\omega]$ ), which simply adds to the signal voltage at the amplifier input, and is amplified at the output; as such, it is completely analogous to the intrinsic detector output noise $\mathcal{S}_{I I}$ of our linear-response detector. In contrast, the current noise source of the volt- age amplifier represents back-action: this fluctuating current (spectral density $\mathcal{S}_{\tilde{I}}[\omega]$ ) flows back across the parallel combination of the source impedance and amplifier input impedance, producing an additional fluctuating voltage at its input. The current noise is thus analogous to the back-action noise $\mathcal{S}_{F F}$ of our generic linearresponse detector.

Putting the above together, the total voltage at the input terminals of the amplifier is

$$
\begin{aligned}
v_{\text {in }, \text { tot }}(t) & =\frac{Z_{\text {in }}}{Z_{\text {in }}+Z_{s}}\left[v_{\text {in }}(t)+\tilde{V}(t)\right]-\frac{Z_{s} Z_{\text {in }}}{Z_{s}+Z_{\text {in }}} \tilde{I}(t) \\
& \simeq v_{\text {in }}(t)+\tilde{V}(t)-Z_{s} \tilde{I}(t) .
\end{aligned}
$$

In the second line, we have taken the usual limit of an ideal voltage amplifier which has an infinite input impedance (i.e., the amplifier draws zero current). The spectral density of the total input voltage fluctuations is thus

$$
\mathcal{S}_{V V, \text { tot }}[\omega]=\mathcal{S}_{v_{\text {in }} \text { in }}[\omega]+\mathcal{S}_{V V, \text { add }}[\omega] .
$$

Here $\mathcal{S}_{v_{\text {in }} v_{\text {in }}}$ is the spectral density of the voltage fluctuations of the input signal $v_{\text {in }}(t)$ and $S_{V V \text {,add }}$ is the amplifier's contribution to the total noise at the input

$$
\mathcal{S}_{V V, \text { add }}[\omega]=\mathcal{S}_{V}^{\tilde{V}}+\left|Z_{s}\right|^{2} \mathcal{S}_{I I}-2 \operatorname{Re}\left[Z_{s}^{*} \mathcal{S}_{V \tilde{I}}\right]
$$

For clarity, we have dropped the frequency index for the spectral densities appearing on the RHS of this equation.

It is useful now to consider a narrow bandwidth input signal at a frequency $\omega$, and ask the following question: if the signal source was simply an equilibrium resistor at a temperature $T_{0}$, how much hotter would it have to be to produce a voltage noise equal to $\mathcal{S}_{V V \text {,tot }}[\omega]$ ? The resulting increase in the source temperature is defined as the noise temperature $T_{N}[\omega]$ of the amplifier and is a convenient measure of the amplifier's added noise. It is standard among engineers to define the noise temperature with the assumption that the initial temperature of the resistor $T_{0} \gg \hbar \omega$. One may then use the classical expression for the thermal noise of a resistor, which yields the definition

$$
2 \operatorname{Re} Z_{s} k_{B} T_{N}[\omega] \equiv \mathcal{S}_{V V \text {,tot }}[\omega] .
$$

Writing $Z_{s}=\left|Z_{s}\right| e^{i \phi}$, we have

$$
2 k_{B} T_{N}=\frac{1}{\cos \phi}\left[\frac{\mathcal{S}_{\tilde{V} \tilde{V}}}{\left|Z_{s}\right|}+\left|Z_{s}\right| \mathcal{S}_{\tilde{I I}}-2 \operatorname{Re}\left(e^{-i \phi} \mathcal{S}_{\tilde{V} \tilde{I}}\right)\right] \text {. }
$$

It is clear from this expression that $T_{N}$ will have a minimum as a function of $\left|Z_{s}\right|$. For $\left|Z_{s}\right|$ too large, the backaction current noise of the amplifier will dominate $T_{N}$, while for $\left|Z_{s}\right|$ too small, the voltage noise of the amplifier (i.e., its intrinsic output noise) will dominate. The situation is completely analogous to that of the position detector of the last section; there we needed to optimize the coupling strength $A$ to balance back-action and intrinsic output noise contributions and thus minimize the 
total added noise. Optimization of the source impedance thus yields a completely classical minimum bound on $T_{N}$,

$$
k_{B} T_{N} \geqslant \sqrt{\mathcal{S}_{V} \tilde{V} \mathcal{S}_{I I} \tilde{I}-\left[\operatorname{Im} \mathcal{S}_{V I}^{\tilde{V}}\right]^{2}}-\operatorname{Re} \mathcal{S}_{V} \tilde{I},
$$

where the minimum is achieved for an optimal source impedance that satisfies

$$
\begin{aligned}
& \left|Z_{s}[\omega]\right|_{\mathrm{opt}}=\sqrt{\mathcal{S}_{\tilde{V} \tilde{V}}[\omega] / \mathcal{S}_{I \tilde{I}}^{\tilde{I}}[\omega]} \equiv Z_{N}, \\
& \left.\sin \phi[\omega]\right|_{\mathrm{opt}}=-\operatorname{Im} \mathcal{S}_{\tilde{V} \tilde{I}}[\omega] / \sqrt{S_{\tilde{V} \tilde{V}}[\omega] \mathcal{S}_{I I I}[\omega]} .
\end{aligned}
$$

The above equations define the so-called noise impedance $Z_{N}$. We stress again that the discussion so far in this section has been completely classical.

\section{Linear-response description}

It is easy to connect the classical description of a voltage amplifier to the quantum-mechanical description of a generic linear-response detector; in fact, all that is needed is a "relabeling" of the concepts and quantities introduced in Sec. V.E when discussing a linear position detector. Thus, the quantum voltage amplifier will be characterized by both an input operator $\hat{Q}$ and an output operator $\hat{V}_{\text {out }}$; these play the roles of $\hat{F}$ and $\hat{I}$ in the position detector, respectively. $\hat{V}_{\text {out }}$ represents the output voltage of the amplifier, while $\hat{Q}$ is the operator which couples to the input signal $v_{\text {in }}(t)$ via a coupling Hamiltonian

$$
\hat{H}_{\mathrm{int}}=v_{\mathrm{in}}(t) \hat{Q} .
$$

In more familiar terms, $\hat{\tilde{I}}_{\text {in }}-d \hat{Q} / d t$ represents the current flowing into the amplifier. ${ }^{17}$ The voltage gain of our amplifier $\lambda_{V}$ will again be given by the Kubo formula of Eq. (4.3), with the substitutions $\hat{F} \rightarrow \hat{Q}, \hat{I} \rightarrow \hat{V}_{\text {out }}$ (we assume these substitutions throughout this section).

We can now easily relate the fluctuations of the input and output operators to the noise sources used to describe the classical voltage amplifier. As usual, symmetrized quantum noise spectral densities $\bar{S}[\omega]$ will play the role of the classical spectral densities $\mathcal{S}[\omega]$ appearing in the classical description. First, as the operator $\hat{Q}$ represents a back-action force, its fluctuations correspond to the amplifier's current noise $\tilde{I}(t)$,

\footnotetext{
${ }^{17}$ Note that one could have instead written the coupling Hamiltonian in the more traditional form $\hat{H}_{\text {int }}(t)=\phi(t) \hat{\tilde{I}}_{\text {in }}$, where $\phi=\int d t^{\prime} v_{\text {in }}\left(t^{\prime}\right)$ is the flux associated with the input voltage. The linear-response results we obtain are exactly the same. We prefer to work with the charge $\hat{Q}$ in order to be consistent with the rest of the text.
}

$$
\mathcal{S}_{I I}[\omega] \leftrightarrow \omega^{2} \bar{S}_{Q Q}[\omega] .
$$

Similarly, the fluctuations in the operator $\hat{V}_{\text {out }}$, when referred back to the amplifier input, will correspond to the voltage noise $\tilde{V}(t)$ discussed above,

$$
\mathcal{S}_{\tilde{V} \tilde{V}}^{\tilde{V}}[\omega] \leftrightarrow \bar{S}_{V_{\text {out }} V_{\text {out }}}[\omega] /\left|\lambda_{V}\right|^{2} .
$$

A similar correspondence holds for the cross correlator of these noise sources,

$$
\mathcal{S}_{\tilde{V} \tilde{I}}[\omega] \leftrightarrow+i \omega \bar{S}_{V_{\text {out }} Q}[\omega] / \lambda_{V} .
$$

To proceed, we need to identify the input and output impedances of the amplifier, and then define its power gain. The first step in this direction is to assume that the output of the amplifier $\left(\hat{V}_{\text {out }}\right)$ is connected to an external circuit via a term

$$
\hat{H}_{\text {int }}^{\prime}=q_{\text {out }}(t) \hat{V}_{\text {out }},
$$

where $\tilde{i}_{\text {out }}=d q_{\text {out }} / d t$ is the current in the external circuit. We may now identify the input and output impedances of the amplifier in terms of the damping at the input and output. Use of the Kubo formulas for conductance and resistance yields [cf. Eq. (2.12) and Eq. (5.48), with the substitutions $\hat{F} \rightarrow \hat{Q}$ and $\hat{I} \rightarrow \hat{V}]$

$$
\begin{aligned}
& 1 / Z_{\text {in }}[\omega]=i \omega \chi_{Q Q}[\omega], \\
& Z_{\text {out }}[\omega]=\chi_{V V}[\omega] /(-i \omega),
\end{aligned}
$$

i.e., $\left\langle\tilde{I}_{\text {in }}\right\rangle_{\omega}=\left(1 / Z_{\text {in }}[\omega]\right) v_{\text {in }}[\omega]$ and $\langle V\rangle_{\omega}=Z_{\text {out }}[\omega] \tilde{i}_{\text {out }}[\omega]$, where the subscript $\omega$ indicates the Fourier transform of a time-dependent expectation value.

We consider throughout this section the case of no reverse gain, $\chi_{Q V_{\text {out }}}=0$. We can define the power gain $G_{P}$ exactly as in Sec. V.E.3 for a linear position detector. $G_{P}$ is defined as the ratio of the power delivered to a load attached to the amplifier output divided by the power drawn by the amplifier, maximized over the impedance of the load. One finds

$$
G_{P}=\left|\lambda_{V}\right|^{2} / 4 \operatorname{Re}\left(Z_{\text {out }}\right) \operatorname{Re}\left(1 / Z_{\text {in }}\right) .
$$

Expressing this in terms of the linear-response coefficients $\lambda_{V V}$ and $\chi_{Q Q}$, we obtain an expression that is completely analogous to Eq. (5.52) for the power gain for a position detector

$$
G_{P}=\left|\lambda_{V}\right|^{2} / 4 \operatorname{Im} \chi_{Q Q} \operatorname{Im} \chi_{V V}
$$

Finally, we define again the effective temperature $T_{\text {eff }}[\omega]$ of the amplifier via Eq. (2.8), and define a quantumlimited voltage amplifier as one that satisfies the idealnoise condition of Eq. (4.17). For such an amplifier, the power gain will be determined by the effective temperature via Eq. (5.56).

Turning to the noise, we calculate the total symmetrized noise at the output port of the amplifier following the same argument used to get the output noise of the position detector [cf. Eq. (5.46)]. As we did in the clas- 
sical approach, we assume that the input impedance of the amplifier is much larger than the source impedance: $Z_{\text {in }} \gg Z_{s}$; we test this assumption for consistency at the end of the calculation. Focusing only on the amplifier contribution to this noise (as opposed to the intrinsic noise of the input signal), and referring this noise back to the amplifier input, we find that the symmetrized quantum noise spectral density describing the added noise of the amplifier $\bar{S}_{V V \text {,add }}[\omega]$ satisfies the same equation we found for a classical voltage amplifier, Eq. (5.73), with each classical spectral density $\mathcal{S}[\omega]$ replaced by the corresponding symmetrized quantum spectral density $\bar{S}[\omega]$ as per Eqs. (5.80)-(5.82).

It follows that the amplifier noise temperature will again be given by Eq. (5.75), and that the optimal noise temperature (after optimizing over the source impedance) will be given by Eq. (5.76). Whereas classically nothing more could be said, quantum mechanically we now get a further bound from the quantum noise constraint of Eq. (4.11) and the requirement of a large power gain. The latter requirement tells us that the voltage gain $\lambda_{V}[\omega]$ and the cross correlator $\bar{S}_{V_{\text {out }} Q}$ must be in phase (cf. Sec. IV.A.4 and Appendix I.3). This in turn means that $\bar{S}_{\tilde{V} \tilde{I}}$ must be purely imaginary. In this case, the quantum noise constraint may be rewritten as

$$
\bar{S}_{\tilde{V} \tilde{V}}[\omega] \bar{S}_{\tilde{I} \tilde{L}}[\omega]-\left[\operatorname{Im} \bar{S}_{\tilde{V} \tilde{I}}\right]^{2} \geqslant(\hbar \omega / 2)^{2} .
$$

Using these results in Eq. (5.76), we find the ultimate quantum limit on the noise temperature, ${ }^{18}$

$$
k_{B} T_{N}[\omega] \geqslant \hbar \omega / 2
$$

As for the position detector, reaching the quantum limit here is not simply a matter of tuning the coupling [i.e., tuning the source impedance $Z_{s}$ to match the noise impedance, cf. Eq. (5.77) and (5.78)]; one also needs to have an amplifier with ideal quantum noise, that is, an amplifier satisfying Eq. (4.17).

Finally, we need to test our initial assumption that $\left|Z_{s}\right| \ll\left|Z_{\text {in }}\right|$, taking $\left|Z_{s}\right|$ to be equal to its optimal value $Z_{N}$. Using the proportionality condition of Eq. (I13) and the fact that we are in the large-power-gain limit $\left(G_{P}[\omega] \gg 1\right)$, we find

\footnotetext{
${ }^{18}$ Note that our definition of the noise temperature $T_{N}$ conforms with that of Devoret and Schoelkopf (2000) and most electrical engineering texts, but is slightly different from that of Caves (1982). Caves assumed the source is initially at zero temperature (i.e., $T_{0}=0$ ), and consequently used the full quantum expression for its equilibrium noise. In contrast, we have assumed that $k_{B} T_{0} \gg \hbar \omega$. The different definition of the noise temperature used by Caves leads to the result $k_{B} T_{N}$ $\geqslant \hbar \omega /(\ln 3)$ as opposed to our Eq. (5.89). We stress that the difference between these results has nothing to do with physics, but only with how one defines the noise temperature.
}

$$
\left|\frac{Z_{N}[\omega]}{\operatorname{Re} Z_{\text {in }}[\omega]}\right|=\left|\frac{\alpha}{\operatorname{Im} \alpha}\right| \frac{\hbar \omega}{4 k_{B} T_{\text {eff }}}=\frac{1}{2 \sqrt{G_{P}[\omega]}} \ll 1 .
$$

It follows that $\left|Z_{N}\right| \ll\left|Z_{\text {in }}\right|$ in the large-power-gain, largeeffective-temperature regime of interest, thus justifying the form of Eq. (5.73). Equation (5.90) is analogous to the case of the displacement detector, where we found that reaching the quantum limit on resonance required the detector-dependent damping to be much weaker than the intrinsic damping of the oscillator [cf. Eq. (5.69)].

Thus, similarly to the situation of the displacement detector, the linear-response approach allows us both to derive rigorously the quantum limit on the noise temperature $T_{N}$ of an amplifier and to state conditions that must be met to reach this limit. To reach the quantumlimited value of $T_{N}$ with a large power gain, one needs both a tuned source impedance $Z_{s}$ and an amplifier that possesses ideal noise properties [cf. Eqs. (4.17) and Eq. (I13)].

\section{Role of noise cross correlations}

Before leaving the topic of a linear voltage amplifier, we pause to note the role of cross correlations in current and voltage noise in reaching the quantum limit. First, note from Eq. (5.78) that in both the classical and quantum treatments the noise impedance $Z_{N}$ of the amplifier will have a reactive part (i.e., $\operatorname{Im} Z_{N} \neq 0$ ) if there are out-of-phase correlations between the amplifier's current and voltage noises (i.e., if $\operatorname{Im} S_{V I} \neq 0$ ). Thus, if such correlations exist, it will not be possible to minimize the noise temperature (and hence reach the quantum limit), if one uses a purely real source impedance $Z_{s}$.

More significantly, note that the final classical expression for the noise temperature $T_{N}$ explicitly involves the real part of the $S_{V I}$ correlator [cf. Eq. (5.76)]. In contrast, we have shown that in the quantum case Re $\bar{S}_{V I}$ must be zero if one wishes to reach the quantum limit while having a large power gain (see Appendix I.3); as such, this quantity does not appear in the final expression for the minimal $T_{N}$. It also follows that to reach the quantum limit while having a large power gain, an amplifier cannot have significant in-phase correlations between its current and voltage noise.

This last statement can be given a heuristic explanation. If there are out-of-phase correlations between current and voltage noise, we can easily make use of these by appropriately choosing our source impedance. However, if there are in-phase correlations between current and voltage noise, we cannot use these simply by tuning the source impedance. We could, however, have used them by implementing feedback in our amplifier. The fact that we have not done this means that these correlations represent a kind of missing information; as a result, we must necessarily miss the quantum limit. In Sec. VI.B, we explicitly give an example of a voltage amplifier which misses the quantum limit due to the presence 
of in-phase current and voltage fluctuations; we show how this amplifier can be made to reach the quantum limit by adding feedback in Appendix $\mathrm{H}$.

\section{G. Near quantum-limited mesoscopic detectors}

Having discussed the origin and precise definition of the quantum limit on the added noise of a linear, phasepreserving amplifier, we now provide a review of work examining whether particular detectors are able in principle to achieve this ideal limit. We focus on the op-amp mode of operation discussed in Sec. V.D, where the detector is only weakly coupled to the system producing the signal to be amplified. As repeatedly stressed, reaching the quantum limit in this case requires the detector to have quantum-ideal noise, as defined by Eq. (4.17). Heuristically, this corresponds to the general requirement of no wasted information: there should be no other quantity besides the detector output that could be monitored to provide information on the input signal (Clerk et al., 2003). We have already given one simple but relevant example of a detector which reaches the amplifier quantum limit: the parametric cavity detector, discussed in Sec. III.B. Here we turn to other more complex detectors.

\section{1. dc superconducting quantum interference device amplifiers}

The dc superconducting quantum interference device (SQUID) is a detector based on a superconducting ring having two Josephson junctions. It can in principle be used as a near quantum-limited voltage amplifier or flux-to-voltage amplifier. Theoretically, this was investigated using a quantum Langevin approach (Koch et al., 1981; Danilov et al., 1983), as well as more rigorously by using perturbative techniques (Averin, 2000b) and mappings to quantum impurity problems (Clerk, 2006). Experiments on SQUIDS have also confirmed their potential for near-quantum-limited operation. Mück et al. (2001) were able to achieve a noise temperature $T_{N}$ approximately 1.9 times the quantum-limited value at an operating frequency of $\omega=2 \pi \times 519 \mathrm{MHz}$. Working at lower frequencies appropriate to gravitational wave detection applications, Vinante et al. (2001) were able to achieve a $T_{N}$ approximately 200 times the quantumlimited value at a frequency $\omega=2 \pi \times 1.6 \mathrm{kHz}$; more recently, the same group achieved a $T_{N}$ approximately ten times the quantum limit at a frequency $\omega=2 \pi$ $\times 1.6 \mathrm{kHz}$ (Falferi et al., 2008). In practice, it can be difficult to achieve the theoretically predicted quantumlimited performance due to spurious heating caused by the dissipation in the shunt resistances used in the SQUID. This effect can be significantly ameliorated, however, by adding cooling fins to the shunts (Wellstood et al., 1994).

\section{Quantum point contact detectors}

A quantum point contact (QPC) is a narrow conducting channel formed in a two-dimensional gas. The current through the constriction is sensitive to nearby charges, and thus the QPC acts as a charge-to-current amplifier. It has been shown theoretically that the QPC can achieve the amplifier quantum limit, both in the regime where transport is due to tunneling (Gurvitz, 1997), and in regimes where the transmission is not small (Aleiner et al., 1997; Levinson, 1997; Korotkov and Averin, 2001; Pilgram and Büttiker, 2002; Clerk et al., 2003). Experimentally, QPCs are in widespread use as detectors of quantum dot qubits. The back-action dephasing of QPC detectors was studied by Buks et al. (1998) and Sprinzak et al. (2000); good agreement was found with the theoretical prediction, confirming that the QPC has quantum-limited back-action noise.

\section{Single-electron transistors and resonant-level detectors}

A metallic single-electron transistor (SET) consists of a small metallic island attached via tunnel junctions to larger source and drain electrodes. Because of Coulomb-blockade effects, the conductance of a SET is sensitive to nearby charges, and hence it acts as a sensitive charge-to-current amplifier. Considerable work has investigated whether metallic SETs can approach the quantum limit in various different operating regimes. Theoretically, the performance of a normal-metal SET in the sequential tunneling regime was studied by Shnirman and Schön (1998), Devoret and Schoelkopf (2000), Makhlin et al. (2000), Aassime et al. (2001), and Johansson et al. $(2002,2003)$. In this regime, where transport is via a sequence of energy-conserving tunnel events, one is far from optimizing the quantum noise constraint of Eq. (4.17), and hence one cannot reach the quantum limit (Shnirman and Schön, 1998; Korotkov, 2001b). If one instead chooses to work with a normal-metal SET in the cotunneling regime (a higher-order tunneling process involving a virtual transition), then one can indeed approach the quantum limit (Averin, 2000a; van den Brink, 2002). However, by virtue of being a higher-order process, the related currents and gain factors are small, impinging on the practical utility of this regime of operation. It is worth noting that while most theory on SETs assume a dc voltage bias, to enhance bandwidth, experiments are usually conducted using the rf-SET configuration (Schoelkopf et al., 1998), where the SET changes the damping of a resonant LC circuit. Korotkov and Paalanen (1999) showed that this mode of operation for a sequential tunneling SET increases the measurementimprecision noise by approximately a factor of 2 . The measurement properties of a normal-metal, sequentialtunneling rf-SET (including back-action) were studied experimentally by Turek et al. (2005).

Measurement using superconducting SETs has also been studied. Clerk et al. (2002) showed that so-called incoherent Cooper-pair tunneling processes in a superconducting SET can have a noise temperature which is approximately a factor of 2 larger than the quantumlimited value. The measurement properties of superconducting SETs biased at a point of incoherent Cooperpair tunneling have been probed recently in experiment (Thalakulam et al., 2004; Naik et al., 2006). 
The quantum measurement properties of phasecoherent noninteracting resonant level detectors have also been studied theoretically (Averin, 2000b; Clerk and Stone, 2004; Mozyrsky et al., 2004; Gavish et al., 2006). These systems are similar to metallic SET, except that the central island only has a single level (as opposed to a continuous density of states), and Coulombblockade effects are typically neglected. These detectors can reach the quantum limit in the regime where the voltage and temperature are smaller than the intrinsic energy broadening of the level due to tunneling. They can also reach the quantum limit in a large-voltage regime that is analogous to the cotunneling regime in a metallic SET (Averin, 2000b; Clerk and Stone, 2004). The influence of dephasing processes on such a detector was studied by Clerk and Stone (2004).

\section{H. Back-action evasion and noise-free amplification}

Having discussed in detail quantum limits on phasepreserving linear amplifiers (i.e., amplifiers which measure both quadratures of a signal equally well), we now return to the situation discussed in Sec. V.A: imagine we wish only to amplify a single quadrature of some timedependent signal. For this case, there need not be any added noise from the measurement. Unlike the case of amplifying both quadratures, Liouville's theorem does not require the existence of any additional degrees of freedom when amplifying a single quadrature: phase space volume can be conserved during amplification simply by contracting the unmeasured quadrature [see Eq. (5.2)]. As no extra degrees of freedom are needed, there need not be any extra noise associated with the amplification process.

Alternatively, single-quadrature detection can take a form similar to a QND measurement, where the backaction does not affect the dynamics of the quantity being measured (Thorne et al., 1978; Braginsky et al., 1980; Caves et al., 1980; Caves, 1982; Braginsky and Khalili, 1992; Bocko and Onofrio, 1996). For concreteness, consider a high- $Q$ harmonic oscillator with position $x(t)$ and resonant frequency $\Omega$. Its motion may be written in terms of quadrature operators defined as in Eq. (3.48),

$$
\hat{x}(t)=\hat{X}_{\delta}(t) \cos (\Omega t+\delta)+\hat{Y}_{\delta}(t) \sin (\Omega t+\delta) .
$$

Here $\hat{x}(t)$ is the Heisenberg-picture position operator of the oscillator. The quadrature operators can be written in terms of the (Schrödinger-picture) oscillator creation and destruction operators as

$$
\begin{aligned}
& \hat{X}_{\delta}(t)=x_{\mathrm{ZPF}}\left(\hat{c} e^{i(\Omega t+\delta)}+\hat{c}^{\dagger} e^{-i(\Omega t+\delta)}\right), \\
& \hat{Y}_{\delta}(t)=-i x_{\mathrm{ZPF}}\left(\hat{c} e^{i(\Omega t+\delta)}-\hat{c}^{\dagger} e^{-i(\Omega t+\delta)}\right) .
\end{aligned}
$$

As previously discussed, the two quadrature amplitude operators $\hat{X}_{\delta}$ and $\hat{Y}_{\delta}$ are canonically conjugate [cf. Eq. (3.49)]. Making a measurement of one quadrature amplitude, say $\hat{X}_{\delta}$, will thus invariably lead to backaction disturbance of the other conjugate quadrature $\hat{Y}_{\delta}$.
However, due to the dynamics of a harmonic oscillator, this disturbance will not affect the measured quadrature at later times. One can already see this from the classical equations of motion. Suppose our oscillator is driven by a time-dependent force $F(t)$ which has appreciable bandwidth only near $\Omega$. We may write this as

$$
F(t)=F_{X}(t) \cos (\Omega t+\delta)+F_{Y}(t) \sin (\Omega t+\delta),
$$

where $F_{X}(t), F_{Y}(t)$ are slowly varying compared to $\Omega$. Using the fact that the oscillator has a high-quality factor $Q=\Omega / \gamma$, one can easily find the equations of motion,

$$
d X_{\delta}(t) / d t=-(\gamma / 2) X_{\delta}(t)-F_{Y}(t) / 2 m \Omega,
$$

$$
d Y_{\delta}(t) / d t=-(\gamma / 2) Y_{\delta}(t)+F_{X}(t) / 2 m \Omega .
$$

Thus, as long as $F_{Y}(t)$ and $F_{X}(t)$ are uncorrelated and sufficiently slow, the dynamics of the two quadratures are completely independent; in particular, if $Y_{\delta}$ is subject to a narrow-bandwidth, noisy force, it is of no consequence to the evolution of $X_{\delta}$. An ideal measurement of $X_{\delta}$ will result in a back-action force having the form of Eq. (5.93) with $F_{Y}(t)=0$, implying that $X_{\delta}(t)$ will be completely unaffected by the measurement.

Not surprisingly, if one can measure and amplify $X_{\delta}$ without any back-action, there need not be any added noise due to the amplification. In such a setup, the only added noise is the measurement-imprecision noise associated with intrinsic fluctuations of the amplifier output. These may be reduced in principle to an arbitrarily small value by simply increasing the amplifier gain (e.g., by increasing the detector-system coupling): in an ideal setup, there is no back-action penalty on the measured quadrature associated with this increase.

The above conclusion can lead to what seems like a contradiction. Imagine we use a back-action evading amplifier to make a "perfect" measurement of $X_{\delta}$ (i.e., negligible added noise). We would then have no uncertainty as to the value of this quadrature. Consequently, we would expect the quantum state of our oscillator to be a squeezed state, where the uncertainty in $X_{\delta}$ is much smaller than $x_{\mathrm{ZPF}}$. However, if there is no back-action acting on $X_{\delta}$, how is the amplifier able to reduce its uncertainty? This seeming paradox can be fully resolved by considering the conditional aspects of an ideal singlequadrature measurement, where one considers the state of the oscillator given a particular measurement history (Ruskov et al., 2005; Clerk et al., 2008).

It is worth stressing that the possibility of amplifying a single quadrature without back-action (and hence without added noise) relies crucially on our oscillator resembling a perfect harmonic oscillator: the oscillator $Q$ must be large, and nonlinearities (which could couple the two quadratures) must be small. In addition, the envelope of the nonvanishing back-action force $F_{X}(t)$ must have a narrow bandwidth. One should further note that a very high-precision measurement of $X_{\delta}$ will produce a very large back-action force $F_{X}$. If the system is not nearly 
perfectly harmonic, then the large amplitude imparted to the conjugate quadrature $Y_{\delta}$ will inevitably leak back into $X_{\delta}$.

Amplifiers or detectors that treat the two signal quadratures differently are known in the quantum optics literature as phase sensitive; we prefer the designation phase nonpreserving since they do not preserve the phase of the original signal. Such amplifiers invariably rely on some internal clock (i.e., an oscillator with a well-defined phase) which breaks time-translation invariance and picks out the phase of the quadrature that will be amplified [i.e., the choice of $\delta$ used to define the two quadratures in Eq. (5.91)]; we see this explicitly in what follows. This leads to an important caveat: even in a situation where the interesting information is in a single signal quadrature, to benefit from using a phasenonpreserving amplifier, we must know in advance the precise phase of this quadrature. If we do not know this phase, we either have to revert to a phase-preserving amplification scheme (and thus be susceptible to added noise) or we would have to develop a sophisticated and high speed quantum feedback scheme to dynamically adapt the measurement to the correct quadrature in real time (Armen et al., 2002). In what follows, we make the above ideas concrete by considering a few examples of quantum phase-nonpreserving amplifiers. ${ }^{19}$

\section{Degenerate parametric amplifier}

Perhaps the simplest example of a phase nonpreserving amplifier is the degenerate parametric amplifier; the classical version of this system was described in Sec. V.A [see Eq. (5.2)]. The setup is similar to the nondegenerate parametric amplifier discussed in Sec. V.C, except that the idler mode is eliminated, and the nonlinearity converts a single pump photon into two signal photons at frequency $\omega_{S}=\omega_{P} / 2$. As we now show, the resulting dynamics causes one signal quadrature to be amplified while the other is attenuated, in such a way that it is not necessary to add extra noise to preserve the canonical commutation relations.

The system Hamiltonian is

$$
\hat{H}_{\mathrm{sys}}=\hbar\left(\omega_{P} \hat{a}_{P}^{\dagger} \hat{a}_{P}+\omega_{S} \hat{a}_{S}^{\dagger} \hat{a}_{S}\right)+i \hbar \eta\left(\hat{a}_{S}^{\dagger} \hat{a}_{S}^{\dagger} \hat{a}_{P}-\hat{a}_{S} \hat{a}_{S} \hat{a}_{P}^{\dagger}\right) .
$$

If the pump is treated classically as before, the analog of Eq. (5.16) is

$$
\hat{V}_{\text {sys }}=i \hbar(\lambda / 2)\left(\hat{a}_{S}^{\dagger} \hat{a}_{S}^{\dagger}-\hat{a}_{S} \hat{a}_{S}\right),
$$

where $\lambda / 2=\eta \psi_{P}$, and the analog of Eq. (5.19) is

$$
\dot{\hat{a}}_{S}=-\left(\kappa_{S} / 2\right) \hat{a}_{S}+\lambda \hat{a}_{S}^{\dagger}-\sqrt{\kappa_{S}} \hat{b}_{S, \text { in }} .
$$

\footnotetext{
${ }^{19}$ One could in principle generalize the linear-response approach of Sec. V to deal with phase-nonpreserving detectors. However, as such detectors are not time-translational invariant, such a description becomes rather cumbersome and is not particularly helpful. We prefer instead to present concrete examples.
}

The dimensionless quadrature operators corresponding to the signal mode are

$$
\hat{x}_{S}=(1 / \sqrt{2})\left(\hat{a}_{S}^{\dagger}+\hat{a}_{S}\right), \quad \hat{y}_{S}=(i / \sqrt{2})\left(\hat{a}_{S}^{\dagger}-\hat{a}_{S}\right),
$$

which obey $\left[\hat{x}_{S}, \hat{y}_{S}\right]=i$. We can define quadrature operators $\hat{X}_{S \text {,in/out }}, \hat{Y}_{S \text {,in/out }}$ corresponding to the input and output fields in a completely analogous manner.

The steady state solution of Eq. (5.97) for the output fields becomes

$$
\hat{X}_{S, \text { out }}=\sqrt{G} \hat{X}_{S, \text { in }}, \quad \hat{Y}_{S, \text { out }}=\hat{Y}_{S, \text { in }} / \sqrt{G},
$$

where the number gain $G$ is given by

$$
G=\left[\left(\lambda+\kappa_{S} / 2\right) /\left(\lambda-\kappa_{S} / 2\right)\right]^{2} .
$$

We thus see clearly that the amplifier treats the two quadratures differently. One quadrature is amplified and the other attenuated, with the result that the commutation relation can be preserved without the necessity of extra degrees of freedom and added noise. Note that the large-amplitude pump mode has played the role of a clock in the degenerate paramp: it is the phase of the pump that picks out which quadrature of the signal will be amplified.

Before ending our discussion here, it is important to stress that while the degenerate parametric amplifier is phase sensitive and has no added noise, it is not an example of back-action evasion [see Caves et al. (1980), footnote on p. 342]. This amplifier is operated in the scattering mode of amplifier operation, a mode where (as discussed in Sec. V.D) back-action is not relevant at all. Recall that in this mode of operation the amplifier input is perfectly impedance matched to the signal source, and the input signal is simply the amplitude of an incident wave on the amplifier input. This mode of operation necessarily requires a strong coupling between the input signal and the amplifier input (i.e., $\left.\left\langle\hat{b}_{S, \text { in }}\right\rangle\right)$. If one instead tried to weakly couple the degenerate parametric amplifier to a signal source, and operate it in the op-amp mode of operation (cf. Sec. V.D), one finds that there is indeed a back-action disturbance of the measured quadrature. We have yet another example which demonstrates that one must be careful to distinguish the op-amp and scattering modes of amplifier operation.

\section{Double-sideband cavity detector}

We now turn to a simple but experimentally relevant detector that is truly back-action evading. We take as our input signal the position $\hat{x}$ of a mechanical oscillator. The amplifier setup we consider is almost identical to the cavity position detector discussed in Sec. III.B.3: we again have a single-sided resonant cavity whose frequency depends linearly on the oscillator's position, with the Hamiltonian given by Eq. (3.11) (with $\hat{z}=\hat{x} / x_{\mathrm{ZPF}}$ ). We showed in Sec. III.B.3 and Appendix E.3 that, by driving the cavity on resonance, it could be used to make a quantum-limited position measurement: one can operate it as a phase-preserving amplifier of the mechanical oscillator's position and achieve the minimum possible 
amount of added noise. To use the same system to make a back-action free measurement of one oscillator quadrature only, one simply uses a different cavity drive. Instead of driving at the cavity resonance frequency $\omega_{c}$, one drives at the two sidebands associated with the mechanical motion (i.e., at frequencies $\omega_{c} \pm \Omega$, where $\Omega$ is as always the frequency of the mechanical resonator). As we see, such a drive results in an effective interaction that couples the cavity to only one quadrature of the oscillator's motion. This setup was first proposed as a means of back-action evasion by Braginsky et al. (1980); further discussion can be found in Caves et al. (1980) and Braginsky and Khalili (1992), as well as in Clerk et al. (2008), which gives a fully quantum treatment and considers conditional aspects of the measurement. In what follows, we sketch the operation of this system following Clerk et al. (2008); details are provided in Appendix E.4.

We start by requiring that our system be in the "goodcavity" limit, where $\omega_{c} \gg \Omega \gg \kappa(\kappa$ is the damping of the cavity mode); we also require the mechanical oscillator to have a high- $Q$ factor. In this regime, the two sidebands associated with the mechanical motion at $\omega_{c} \pm \Omega$ are well separated from the main cavity resonance at $\omega_{c}$. Making a single-quadrature measurement requires that one drives the cavity equally at the two sideband frequencies. The amplitude of the driving field $\bar{b}_{\text {in }}$ entering the cavity will be chosen to have the form

$$
\begin{aligned}
\bar{b}_{\text {in }}(t) & =-\frac{i \sqrt{\dot{N}}}{4}\left(e^{i \delta} e^{-i\left(\omega_{c}-\Omega\right) t}-e^{-i \delta} e^{-i\left(\omega_{c}+\Omega\right) t}\right) \\
& =\frac{\sqrt{\dot{N}}}{2} \sin (\Omega t+\delta) e^{-i \omega_{c} t} .
\end{aligned}
$$

Here $\dot{N}$ is the photon-number flux associated with the cavity drive (see Appendix E for more details on how to properly include a drive using input-output theory). Such a drive could be produced by taking a signal at the cavity resonance frequency and amplitude-modulating it at the mechanical frequency.

To understand the effect of this drive, note that it sends the cavity both photons with frequency $\omega_{c}-\Omega$ and photons with frequency $\omega_{c}+\Omega$. The first kind of drive photon can be converted to a cavity photon if a quantum is absorbed from the mechanical oscillator; the second kind of drive photon can be converted to a cavity photon if a quantum is emitted to the mechanical oscillator. The result is that we can create a cavity photon by either absorbing or emitting a mechanical oscillator quantum. If there is a well-defined relative phase of $e^{i 2 \delta}$ between the two kinds of drive photons, we would expect the double-sideband drive to yield an effective cavityoscillator interaction of the form

$$
V_{\mathrm{eff}} \propto \sqrt{\dot{N}}\left[\hat{a}^{\dagger}\left(e^{i \delta} \hat{c}+e^{-i \delta} \hat{c}^{\dagger}\right)+\text { H.c. }\right]
$$

$$
\propto \sqrt{\dot{N}}\left(\hat{a}+\hat{a}^{\dagger}\right) \hat{X}_{\delta}
$$

This is exactly what is found in a full calculation (see Appendix E.4). Note that we have written the interaction in an interaction picture in which the fast oscillations of the cavity and oscillator operators have been removed. In the second line, we have made use of Eqs. (5.92) to show that the effective interaction involves only the $\hat{X}_{\delta}$ oscillator quadrature.

We thus see from Eq. (5.102b) that the cavity is coupled only to the oscillator $X_{\delta}$ quadrature. As shown rigorously in Appendix E.3, the result is that the system only measures and amplifies this quadrature: the light leaving the cavity has a signature of $\hat{X}_{\delta}$ but not of $\hat{Y}_{\delta}$. Further, Eq. (5.102b) implies that the cavity operator $\sqrt{\dot{N}}\left(\hat{a}+\hat{a}^{\dagger}\right)$ will act as a noisy force on the $Y_{\delta}$ quadrature. While this will cause a back-action heating of $Y_{\delta}$, it will not affect the measured quadrature $X_{\delta}$. We thus have a true back-action-evading amplifier: the cavity output light lets one measure $X_{\delta}$ free from any back-action effect. Note that, in deriving Eq. (5.102a), we have used the fact that the cavity operators have fluctuations in a narrow bandwidth $\sim \kappa \ll \Omega$ : the back-action force noise is slow compared to the oscillator frequency. If this were not the case, we could still have a back-action heating of the measured $X_{\delta}$ quadrature. Such effects, arising from a nonzero ratio $\kappa / \Omega$, are treated in Clerk et al. (2008).

Finally, as there is no back-action on the measured $X_{\delta}$ quadrature, the only added noise of the amplification scheme is measurement-imprecision noise (e.g., shot noise in the light leaving the cavity). This added noise can be made arbitrarily small by increasing the gain of the detector by, for example, increasing the strength of the cavity drive $\dot{N}$. In a real system where $\kappa / \omega_{M}$ is nonzero, the finite bandwidth of the cavity number fluctuations leads to a small back-action on the $X_{\delta}$. As a result, one cannot make the added noise arbitrarily small, as too large a cavity drive will heat the measured quadrature. Nonetheless, for a sufficiently small ratio $\kappa / \omega_{M}$, one can still beat the standard quantum limit on the added noise (Clerk et al., 2008).

\section{Stroboscopic measurements}

With sufficiently high bandwidth it should be possible to do stroboscopic measurements synchronized with the oscillator motion, which could allow one to go below the standard quantum limit in one quadrature of motion (Caves et al., 1980; Braginsky and Khalili, 1992). To understand this idea, imagine an extreme form of phasesensitive detection in which a Heisenberg microscope makes a strong high-resolution measurement which projects the oscillator onto a state of well-defined position $X_{0}$ at time $t=0$, 


$$
\Psi_{X_{0}}(t)=\sum_{n=0}^{\infty} a_{n} e^{-i(n+1 / 2) \Omega t}|n\rangle,
$$

where the coefficients obey $a_{n}=\left\langle n \mid X_{0}\right\rangle$. Because the position is well defined the momentum is extremely uncertain. (Equivalently, the momentum kick delivered by the back-action of the microscope makes the oscillator momentum uncertain.) Thus the wave packet quickly spreads out and the position uncertainty becomes large. However, because of the special feature that the harmonic oscillator levels are evenly spaced, we can see from Eq. (5.103) that the wave packet re-assembles itself precisely once each period of oscillation because $e^{i n \Omega t}$ $=1$ for every integer $n$. (At half periods, the packet reassembles at position $-X_{0}$.) Hence stroboscopic measurements made once (or twice) per period will be backaction evading and can go below the standard quantum limit. The only limitations will be the finite anharmonicity and damping of the oscillator. Note that the possibility of using mesoscopic electron detectors to perform stroboscopic measurements has recently received attention (Jordan and Büttiker, 2005b; Ruskov et al., 2005).

\section{BOSONIC SCATTERING DESCRIPTION OF A TWO-PORT AMPLIFIER}

In this section, we return again to the topic of Sec. V.F, quantum limits on a quantum voltage amplifier. We now discuss the physics in terms of the bosonic voltage amplifier first introduced in Sec. V.D. Recall that in that section we demonstrated that the standard Haus-Caves derivation of the quantum limit was not directly relevant to the usual weak-coupling op-amp mode of amplifier operation, a mode where the input signal is not simply the amplitude of a wave incident on the amplifier. In this section, we expand upon that discussion, giving an explicit discussion of the differences between the op-amp description of an amplifier presented in Sec. V.E, and the scattering description often used in the quantum optics literature (Grassia, 1998; Courty et al., 1999). We see that what one means by back-action and added noise are not the same in the two descriptions! Further, even though an amplifier may reach the quantum limit when used in the scattering mode (i.e., its added noise is as small as allowed by commutation relations), it can nonetheless fail to achieve the quantum limit when used in the op-amp mode. Finally, the discussion here will also allow us to highlight important aspects of the quantum limit not easily discussed in the more general context of Sec. IV.

\section{A. Scattering versus op-amp representations}

In the bosonic scattering approach, a generic linear amplifier is modeled as a set of coupled bosonic modes. To make matters concrete, we consider the specific case of a voltage amplifier with distinct input and output ports, where each port is a semi-infinite transmission line (see Fig. 9). We start by recalling that a quantum trans- mission line can be described as a set of noninteracting bosonic modes (see Appendix D for a quick review). Denoting the input transmission line with an $a$ and the output transmission line with a $b$, the current and voltage operators in these lines may be written as

$$
\begin{aligned}
& \hat{V}_{q}(t)=\int_{0}^{\infty} \frac{d \omega}{2 \pi}\left(\hat{V}_{q}[\omega] e^{-i \omega t}+\text { H.c. }\right), \\
& \hat{I}_{q}(t)=\sigma_{q} \int_{0}^{\infty} \frac{d \omega}{2 \pi}\left(\hat{I}_{q}[\omega] e^{-i \omega t}+\text { H.c. }\right),
\end{aligned}
$$

with

$$
\begin{aligned}
& \hat{V}_{q}[\omega]=\sqrt{\frac{\hbar \omega}{2} Z_{q}}\left(\hat{q}_{\text {in }}[\omega]+\hat{q}_{\text {out }}[\omega]\right), \\
& \hat{I}_{q}[\omega]=\sqrt{\frac{\hbar \omega}{2 Z_{q}}}\left(\hat{q}_{\text {in }}[\omega]-\hat{q}_{\text {out }}[\omega]\right) .
\end{aligned}
$$

Here $q$ can be equal to $a$ or $b$, and we have $\sigma_{a}=1$, $\sigma_{b}=-1$. The operators $\hat{a}_{\text {in }}[\omega], \hat{a}_{\text {out }}[\omega]$ are bosonic annihilation operators; $\hat{a}_{\text {in }}[\omega]$ describes an incoming wave in the input transmission line (i.e., incident on the amplifier) having frequency $\omega$, while $\hat{a}_{\text {out }}[\omega]$ describes an outgoing wave with frequency $\omega$. The operators $\hat{b}_{\text {in }}[\omega]$ and $\hat{b}_{\text {out }}[\omega]$ describe analogous waves in the output transmission line. We can think of $\hat{V}_{a}$ as the input voltage to our amplifier and $\hat{V}_{b}$ as the output voltage. Similarly, $\hat{I}_{a}$ is the current drawn by the amplifier at the input and $\hat{I}_{b}$ is the current drawn at the output of the amplifier. Finally, $Z_{a}$ $\left(Z_{b}\right)$ is the characteristic impedance of the input (output) transmission line.

As we have seen, amplification invariably requires additional degrees of freedom. Thus, to amplify a signal at a particular frequency $\omega$, there will be $2 N$ bosonic modes involved, where the integer $N$ is necessarily larger than 2. Four of these modes are simply the frequency- $\omega$ modes in the input and output lines (i.e., $\hat{a}_{\text {in }}[\omega], \hat{a}_{\text {out }}[\omega], \hat{b}_{\text {in }}[\omega]$, and $\left.\hat{b}_{\text {out }}[\omega]\right)$. The remaining $2(N$ -2) modes describe auxiliary degrees of freedom involved in the amplification process; these additional modes could correspond to frequencies different from the signal frequency $\omega$. The auxiliary modes can also be divided into incoming and outgoing modes. It is thus convenient to represent them as additional transmission lines attached to the amplifier; these additional lines could be semi-infinite or could be terminated by active elements.

\section{Scattering representation}

In general, our generic two-port bosonic amplifier will be described by an $N \times N$ scattering matrix, which determines the relation between the outgoing and incoming mode operators. The form of this matrix is constrained by the requirement that the output modes obey the usual canonical bosonic commutation relations. It is con- 
venient to express the scattering matrix in a form which only involves the input and output lines explicitly,

$$
\left(\begin{array}{l}
\hat{a}_{\text {out }}[\omega] \\
\hat{b}_{\text {out }}[\omega]
\end{array}\right)=\left(\begin{array}{ll}
s_{11}[\omega] & s_{12}[\omega] \\
s_{21}[\omega] & s_{22}[\omega]
\end{array}\right)\left(\begin{array}{l}
\hat{a}_{\text {in }}[\omega] \\
\hat{b}_{\text {in }}[\omega]
\end{array}\right)+\left(\begin{array}{c}
\hat{\mathcal{F}}_{a}[\omega] \\
\hat{\mathcal{F}}_{b}[\omega]
\end{array}\right)
$$

Here $\hat{\mathcal{F}}_{a}[\omega]$ and $\hat{\mathcal{F}}_{b}[\omega]$ are each an unspecified linear combination of the incoming auxiliary modes introduced above. They thus describe noise in the outgoing modes of the input and output transmission lines which arises from the auxiliary modes involved in the amplification process. Note the similarity between Eqs. (6.3) and (5.7) for the simple one-port bosonic amplifier considered in Sec. V.B.

In the quantum optics literature, one typically views Eq. (6.3) as the defining equation of the amplifier; we call this the scattering representation of our amplifier. The representation is best suited to the scattering mode of amplifier operation described in Sec. V.D. In this mode of operation, the experimentalist ensures that $\left\langle\hat{a}_{i n}[\omega]\right\rangle$ is precisely equal to the signal to be amplified, irrespective of what is coming out of the amplifier. Similarly, the output signal from the amplifier is the amplitude of the outgoing wave in the output line, $\left\langle\hat{b}_{\text {out }}[\omega]\right\rangle$. If we focus on $\hat{b}_{\text {out }}$, we have precisely the same situation as described in Sec. 5.10, where we presented the HausCaves derivation of the quantum limit [see Eq. (5.7)]. It follows that in the scattering mode of operation the matrix element $s_{21}[\omega]$ represents the gain of our amplifier at frequency $\omega,\left|s_{21}[\omega]\right|^{2}$ the corresponding "photonnumber gain," and $\hat{\mathcal{F}}_{b}$ the added noise operator of the amplifier. The operator $\hat{\mathcal{F}}_{a}$ represents the back-action noise in the scattering mode of operation; this backaction has no effect on the added noise of the amplifier in the scattering mode.

Similar to Sec. V.B, one can now apply the standard argument of Haus and Mullen (1962) and Caves (1982) to our amplifier. This argument tells us that since the "out" operators must have the same commutation relations as the "in" operators, the added noise $\hat{\mathcal{F}}_{b}$ cannot be arbitrarily small in the large-gain limit (i.e., $\left|s_{21}\right| \gg 1$ ). Note that this version of the quantum limit on the added noise has nothing to do with back-action. As discussed, this is perfectly appropriate for the scattering mode of operation, as in this mode the experimentalist ensures that the signal going into the amplifier is completely independent of whatever is coming out of the amplifier. This mode of operation could be realized in timedependent experiments, where a pulse is launched at the amplifier. This mode is not realized in most weakcoupling amplification experiments, where the signal to be amplified is not identical to an incident wave amplitude.

\section{Op-amp representation}

In the usual op-amp amplifier mode of operation (described in Sec. IV), the input and output signals are not simply incoming and outgoing wave amplitudes; thus, the scattering representation is not an optimal description of our amplifier. The system we are describing here is a voltage amplifier: thus, in the op-amp mode, the experimentalist would ensure that the voltage at the end of the input line $\left(\hat{V}_{a}\right)$ is equal to the signal to be amplified, and would read out the voltage at the end of the output transmission line $\left(\hat{V}_{b}\right)$ as the output of the amplifier. From Eq. (6.1a), we see that this implies that the amplitude of the wave going into the amplifier, $a_{\text {in }}$, will depend on the amplitude of the wave exiting the amplifier, $a_{\text {out }}$.

Thus, if we want to use our amplifier as a voltage amplifier, we want to find a description that is more tailored to our needs than the scattering representation of Eq. (6.3). This can be found by simply re-expressing the scattering matrix relation of Eq. (6.3) in terms of voltages and currents. The result will be what we term the op amp representation of our amplifier, a representation which is standard in the discussion of classical amplifiers [see, e.g., Boylestad and Nashelsky (2006)]. In this representation, one views $\hat{V}_{a}$ and $\hat{I}_{b}$ as inputs to the amplifier: $\hat{V}_{a}$ is set by whatever we connect to the amplifier input, while $\hat{I}_{b}$ is set by whatever we connect to the amplifier output. In contrast, the outputs of our amplifier are the voltage in the output line, $\hat{V}_{b}$, and the current drawn by the amplifier at the input, $\hat{I}_{a}$. Note that this interpretation of voltages and currents is identical to the way we viewed the voltage amplifier in the linearresponse and quantum noise treatment of Sec. V.F.

Using Eqs. (6.1a) and (6.1b) and suppressing frequency labels for clarity, Eq. (6.3) may be written explicitly in terms of the voltages and current in the input $\left(\hat{V}_{a}, \hat{I}_{a}\right)$ and output $\left(\hat{V}_{b}, \hat{I}_{b}\right)$ transmission lines,

$$
\left(\begin{array}{c}
\hat{V}_{b} \\
\hat{I}_{a}
\end{array}\right)=\left(\begin{array}{cc}
\lambda_{V} & -Z_{\text {out }} \\
\frac{1}{Z_{\text {in }}} & \lambda_{I}^{\prime}
\end{array}\right)\left(\begin{array}{c}
\hat{V}_{a} \\
\hat{I}_{b}
\end{array}\right)+\left(\begin{array}{c}
\lambda_{V} \cdot \hat{\tilde{V}} \\
\hat{\tilde{I}}
\end{array}\right) .
$$

The coefficients in the above matrix are completely determined by the scattering matrix of Eq. (6.3) [see Eqs. (6.7) below]; moreover, they are familiar from the discussion of a voltage amplifier in Sec. V.F. $\lambda_{V}[\omega]$ is the voltage gain of the amplifier, $\lambda_{I}^{\prime}[\omega]$ is the reverse current gain of the amplifier, $Z_{\text {out }}$ is the output impedance, and $Z_{\text {in }}$ is the input impedance. The last term on the RHS of Eq. (6.4) describes the two familiar kinds of amplifier noise. $\hat{\tilde{V}}$ is the usual voltage noise of the amplifier (referred back to the amplifier input), while $\hat{\tilde{I}}$ is the usual current noise of the amplifier. Recall that in this standard description of a voltage amplifier (see Sec. V.F) $\tilde{I}$ represents the back-action of the amplifier: the system producing the input signal responds to these current 
fluctuations, resulting in an additional fluctuation in the input signal going into the amplifier. Similarly, $\lambda_{V} \tilde{V}$ represents the intrinsic output noise of the amplifier: this contribution to the total output noise does not depend on properties of the input signal. Note that we are using a sign convention where a positive $\left\langle\hat{I}_{a}\right\rangle$ indicates a current flowing into the amplifier at its input, while a positive $\left\langle\hat{I}_{b}\right\rangle$ indicates a current flowing out of the amplifier at its output. Also note that the operators $\hat{V}_{a}$ and $\hat{I}_{b}$ on the RHS of Eq. (6.4) will have noise; this noise is entirely due to the systems attached to the input and output of the amplifier, and as such should not be included in what we call the added noise of the amplifier.

Additional important properties of our amplifier follow immediately from quantities in the op-amp representation. As discussed in Sec. V.E, the most important measure of gain in our amplifier is the dimensionless power gain. This is the ratio between power dissipated at the output to that dissipated at the input, taking the output current $I_{B}$ to be $V_{B} / Z_{\text {out }}$,

$$
G_{P} \equiv \frac{\left(\lambda_{V}\right)^{2}}{4} \frac{Z_{\text {in }}}{Z_{\text {out }}}\left(1+\frac{\lambda_{V} \lambda_{I}^{\prime}}{2} \frac{Z_{\text {in }}}{Z_{\text {out }}}\right)^{-1} \text {. }
$$

Another important quantity is the loaded input impedance: what is the input impedance of the amplifier in the presence of a load attached to the output? In the presence of reverse current gain $\lambda_{I}^{\prime} \neq 0$, the input impedance will depend on the output load. Taking the load impedance to be $Z_{\text {load }}$, some simple algebra yields

$$
1 / Z_{\text {in,loaded }}=1 / Z_{\text {in }}+\lambda_{I}^{\prime} \lambda_{V} /\left(Z_{\text {load }}+Z_{\text {out }}\right) .
$$

It is of course undesirable to have an input impedance which depends on the load. Thus, we see yet again that it is undesirable to have appreciable reverse gain in our amplifier (cf. Sec. IV.A.2).

\section{Converting between representations}

After some straightforward algebra we now express the op-amp parameters appearing in Eq. (6.4) in terms of the scattering matrix appearing in Eq. (6.3),

$$
\begin{aligned}
& \lambda_{V}=2 \sqrt{\frac{Z_{b}}{Z_{a}}} \frac{s_{21}}{D}, \\
& \lambda_{I}^{\prime}=2 \sqrt{\frac{Z_{b}}{Z_{a}}} \frac{s_{12}}{D}, \\
& Z_{\text {out }}=Z_{b} \frac{\left(1+s_{11}\right)\left(1+s_{22}\right)-s_{12} s_{21}}{D}, \\
& \frac{1}{Z_{\text {in }}}=\frac{1}{Z_{a}} \frac{\left(1-s_{11}\right)\left(1-s_{22}\right)-s_{12} s_{21}}{D},
\end{aligned}
$$

where all quantities are evaluated at the same frequency $\omega$, and $D$ is defined as

$$
D=\left(1+s_{11}\right)\left(1-s_{22}\right)+s_{12} s_{21} .
$$

Further, the voltage and current noises in the op-amp representation are simple linear combinations of the noises $\hat{\mathcal{F}}_{a}$ and $\hat{\mathcal{F}}_{b}$ appearing in the scattering representation,

$$
\left(\begin{array}{c}
\hat{\tilde{V}} \\
Z_{a} \cdot \hat{\tilde{I}}
\end{array}\right)=\sqrt{2 \hbar \omega Z_{a}}\left(\begin{array}{cc}
-\frac{1}{2} & \frac{1+s_{11}}{2 s_{21}} \\
\frac{s_{22}-1}{D} & -\frac{s_{12}}{D}
\end{array}\right)\left(\begin{array}{c}
\hat{\mathcal{F}}_{a} \\
\hat{\mathcal{F}}_{b}
\end{array}\right) .
$$

Again, all quantities above are evaluated at frequency $\omega$.

Equation (6.9) immediately leads to an important conclusion and caveat: what one calls the "back-action" and "added noise" in the scattering representation (i.e., $\mathcal{F}_{a}$ and $\mathcal{F}_{b}$ ) are not the same as the "back-action" and "added noise" defined in the usual op-amp representation. For example, the op-amp back-action $\hat{\tilde{I}}$ does not in general coincide with $\hat{\mathcal{F}}_{a}$, the back-action in the scattering picture. If we are indeed interested in using our amplifier as a voltage amplifier, we are interested in the total added noise of our amplifier as defined in the opamp representation. As we saw in Sec. V.F [cf. Eq. (5.71)], this quantity involves both the noises $\hat{\tilde{I}}$ and $\hat{\tilde{V}}$. We thus see explicitly something discussed in Sec. V.D: it is dangerous to make conclusions about how an amplifier behaves in the op-amp mode of operation based on its properties in the scattering mode of operation. As we see, even though an amplifier is ideal in the scattering mode (i.e., $\mathcal{F}_{a}$ as small as possible), it can nonetheless fail to reach the quantum limit in the op-amp mode of operation.

In what follows, we calculate the op-amp noises $\hat{\tilde{V}}$ and $\hat{\tilde{I}}$ in a minimal bosonic voltage amplifier, and show explicitly how this description is connected to the more general linear-response treatment of Sec. V.F. However, before proceeding, it is worth noting that Eqs. (6.7a), (6.7b), (6.7c), and (6.7d) are themselves completely consistent with linear-response theory. Using linear response, one would calculate the op-amp parameters $\lambda_{V}$, $\lambda_{I}^{\prime}, Z_{\text {in }}$, and $Z_{\text {out }}$ using Kubo formulas [cf. Eqs. (5.84) and (5.85) and the discussion following Eq. (5.79)]. These in turn would involve correlation functions of $\hat{I}_{a}$ and $\hat{V}_{b}$ evaluated at zero coupling to the amplifier input and output. Zero coupling means that there is no input voltage to the amplifier (i.e., a short circuit at the amplifier input, $\hat{V}_{a}=0$ ) and there is nothing at the amplifier output drawing current (i.e., an open circuit at the amplifier output, $\hat{I}_{b}=0$ ). Equation (6.4) tells us that in this case $\hat{V}_{b}$ and $\hat{I}_{a}$ reduce to the noise operators $\lambda_{V} \hat{\tilde{V}}$ and $\hat{\tilde{I}}$, respectively. Using the fact that the commutators of $\hat{\mathcal{F}}_{a}$ and $\hat{\mathcal{F}}_{b}$ are completely determined by the scattering matrix [cf. Eq. (6.3)], we verify explicitly in Appendix I.5 that the Kubo formulas yield the same results for the op-amp 
gains and impedances as Eqs. (6.7a), (6.7b), (6.7c), and (6.7d) above.

\section{B. Minimal two-port scattering amplifier}

\section{Scattering versus op-amp quantum limit}

In this section we demonstrate that an amplifier which is ideal and minimally complex when used in the scattering operation mode fails, when used as a voltage opamp, to have a quantum-limited noise temperature. The system we look at is similar to the amplifier considered by Grassia (1998), though our conclusions are somewhat different than those found there.

In the scattering representation, one might guess that an ideal amplifier would be one where there are no reflections of signals at the input and output, and no way for incident signals at the output port to reach the input. In this case, Eq. (6.3) takes the form

$$
\left(\begin{array}{l}
\hat{a}_{\text {out }} \\
\hat{b}_{\text {out }}
\end{array}\right)=\left(\begin{array}{cc}
0 & 0 \\
\sqrt{G} & 0
\end{array}\right)\left(\begin{array}{l}
\hat{a}_{\text {in }} \\
\hat{b}_{\text {in }}
\end{array}\right)+\left(\begin{array}{c}
\hat{\mathcal{F}}_{a} \\
\hat{\mathcal{F}}_{b}
\end{array}\right),
$$

where we have defined $\sqrt{G} \equiv s_{21}$. All quantities above should be evaluated at the same frequency $\omega$; for clarity, we omit the explicit $\omega$ dependence of quantities throughout this section.

Turning to the op-amp representation, the above equation implies that our amplifier has no reverse gain, and that the input and output impedances are simply given by the impedances of the input and output transmission lines. From Eqs. (6.7), we have

$$
\begin{aligned}
& \lambda_{V}=2 \sqrt{Z_{b} G / Z_{a}}, \\
& \lambda_{I}^{\prime}=0, \\
& Z_{\text {out }}=Z_{b}, \\
& 1 / Z_{\text {in }}=1 / Z_{a} .
\end{aligned}
$$

We immediately see that our amplifier looks less ideal as an op-amp. The input and output impedances are the same as those of the input and output transmission line. However, for an ideal op-amp, we would have liked $Z_{\text {in }} \rightarrow \infty$ and $Z_{\text {out }} \rightarrow 0$.

Also of interest are the expressions for the amplifier noises in the op-amp representation

$$
\left(\begin{array}{c}
\hat{\tilde{V}} \\
Z_{a} \cdot \hat{\tilde{I}}
\end{array}\right)=-\sqrt{2 \hbar \omega Z_{a}}\left(\begin{array}{cc}
\frac{1}{2} & -\frac{1}{2 \sqrt{G}} \\
1 & 0
\end{array}\right)\left(\begin{array}{c}
\hat{\mathcal{F}}_{a} \\
\hat{\mathcal{F}}_{b}
\end{array}\right) .
$$

As $s_{12}=0$, the back-action noise is the same in both the op-amp and scattering representations: it is determined completely by the noise operator $\hat{\mathcal{F}}_{a}$. However, the voltage noise (i.e., the intrinsic output noise) involves both $\hat{\mathcal{F}}_{a}$ and $\hat{\mathcal{F}}_{b}$. We thus have the unavoidable consequence that there will be correlations in $\hat{\tilde{I}}$ and $\hat{\tilde{V}}$. Note that from basic linear-response theory we know that there must be

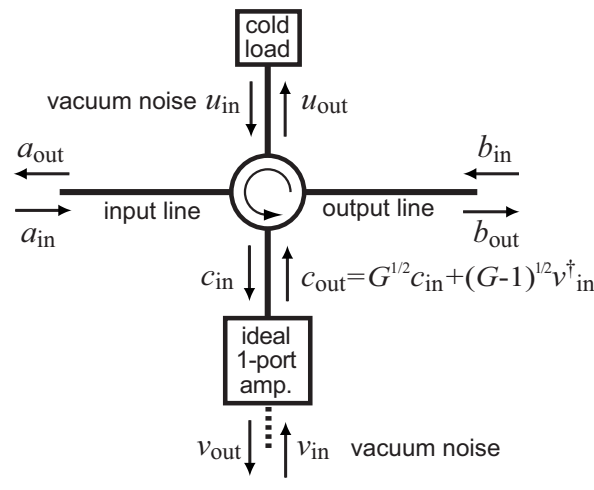

FIG. 13. Schematic of a minimal two-port amplifier which reaches the quantum limit in the scattering mode of operation, but misses the quantum limit when used as a weakly coupled op-amp. See text for further description.

some correlations between $\hat{\tilde{I}}$ and $\hat{\tilde{V}}$ if there is to be gain [i.e., $\lambda_{V}$ is given by a Kubo formula involving these operators, see Eq. (4.3)].

To make further progress, we note again that commutators of the noise operators $\hat{\mathcal{F}}_{a}$ and $\hat{\mathcal{F}}_{b}$ are completely determined by Eq. (6.10) and the requirement that the output operators obey canonical commutation relations. We thus have

$$
\begin{aligned}
& {\left[\hat{\mathcal{F}}_{a}, \hat{\mathcal{F}}_{a}^{\dagger}\right]=1,} \\
& {\left[\hat{\mathcal{F}}_{b}, \hat{\mathcal{F}}_{b}^{\dagger}\right]=1-|G|,} \\
& {\left[\hat{\mathcal{F}}_{a}, \hat{\mathcal{F}}_{b}\right]=\left[\hat{\mathcal{F}}_{a}, \hat{\mathcal{F}}_{b}^{\dagger}\right]=0 .}
\end{aligned}
$$

We will be interested in the limit of a large power gain, which requires $|G| \gg 1$. A minimal solution to the above equations would be to have the noise operators determined by two independent (i.e., mutually commuting) auxiliary input mode operators $u_{i n}$ and $v_{i n}^{\dagger}$,

$$
\begin{aligned}
& \hat{\mathcal{F}}_{a}=\hat{u}_{\text {in }}, \\
& \hat{\mathcal{F}}_{b}=\sqrt{|G|-1} \hat{v}_{\text {in }}^{\dagger} .
\end{aligned}
$$

Further, to minimize the noise of the amplifier, we take the operating state of the amplifier to be the vacuum for both these modes. With these choices, our amplifier is in exactly the minimal form described by Grassia (1998): an input and output line coupled to a negative resistance box and an auxiliary "cold load" via a four-port circulator (see Fig. 13). The negative resistance box is nothing but the single-mode bosonic amplifier discussed in Sec. V.B; an explicit realization of this element would be the parametric amplifier discussed in Sec. V.C. The "cold load" is a semi-infinite transmission line which models dissipation due to a resistor at zero temperature (i.e., its noise is vacuum noise, cf. Appendix D). 
Note that within the scattering picture one would conclude that our amplifier is ideal: in the large-gain limit, the noise added by the amplifier to $\hat{b}_{\text {out }}$ corresponds to a single quantum at the input

$$
\left\langle\left\{\hat{\mathcal{F}}_{b}, \hat{\mathcal{F}}_{b}^{\dagger}\right\}\right\rangle /|G|=[(|G|-1) /|G|]\left\langle\left\{\hat{v}_{\text {in }}^{\dagger}, \hat{v}_{\text {in }}\right\}\right\rangle \rightarrow 1 .
$$

This, however, is not the quantity which interests us: as we want to use this system as a voltage op-amp, we want to know if the noise temperature defined in the op-amp picture is as small as possible. We are also usually interested in the case of a signal that is weakly coupled to our amplifier; here weak coupling means that the input impedance of the amplifier is much larger than the impedance of the signal source (i.e., $Z_{\text {in }} \gg Z_{s}$ ). In this limit, the amplifier only slightly increases the total damping of the signal source.

To address whether we can reach the op-amp quantum limit in the weak-coupling regime, we can make use of the results of the general theory presented in Sec. V.F. In particular, we need to check whether the quantum noise constraint of Eq. (5.88) is satisfied, as this is a prerequisite for reaching the (weak-coupling) quantum limit. Thus, we need to calculate the symmetrized spectral densities of the current and voltage noises, and their cross correlation. It is easy to confirm from the definitions of Eqs. (6.1a) and (6.1b) that these quantities take the form

$$
\begin{aligned}
& \bar{S}_{V V}[\omega]=\left\langle\left\{\hat{\tilde{V}}[\omega], \hat{\tilde{V}}^{\dagger}\left(\omega^{\prime}\right)\right\}\right\rangle / 4 \pi \delta\left(\omega-\omega^{\prime}\right), \\
& \bar{S}_{I I}[\omega]=\left\langle\left\{\hat{\tilde{I}}[\omega], \hat{\tilde{I}}^{\dagger}\left(\omega^{\prime}\right)\right\}\right\rangle / 4 \pi \delta\left(\omega-\omega^{\prime}\right), \\
& \bar{S}_{V I}[\omega]=\left\langle\left\{\hat{\tilde{V}}[\omega], \hat{\tilde{I}}^{\dagger}\left(\omega^{\prime}\right)\right\}\right\rangle / 4 \pi \delta\left(\omega-\omega^{\prime}\right) .
\end{aligned}
$$

The expectation values here are over the operating state of the amplifier; we have chosen this state to be the vacuum for the auxiliary mode operators $\hat{u}_{\text {in }}$ and $\hat{v}_{\text {in }}$ to minimize the noise.

Taking $\left|s_{21}\right| \gg 1$, and using Eqs. (6.14) and (6.15), we have

$$
\begin{aligned}
& \bar{S}_{V V}[\omega]=\hbar \omega Z_{a}\left(\sigma_{u u}+\sigma_{v v}\right) / 4=\hbar \omega Z_{a} / 2, \\
& \bar{S}_{I I}[\omega]=\hbar \omega \sigma_{u u} / Z_{a}=\hbar \omega / Z_{a}, \\
& \bar{S}_{V I}[\omega]=\hbar \omega \sigma_{u u} / 2=\hbar \omega / 2,
\end{aligned}
$$

where we have defined

$$
\sigma_{a b} \equiv\left\langle\hat{a} \hat{b}^{\dagger}+\hat{b}^{\dagger} \hat{a}\right\rangle
$$

and have used the fact that there cannot be any correlations between the operators $u$ and $v$ in the vacuum state (i.e., $\left\langle\hat{u} \hat{v}^{\dagger}\right\rangle=0$ ).

It follows immediately from the above equations that our minimal amplifier does not optimize the quantum noise constraint of Eq. (5.88),

$$
\bar{S}_{V V}[\omega] \bar{S}_{I I}[\omega]-\left[\operatorname{Im} \bar{S}_{V I}\right]^{2}=2 \times(\hbar \omega / 2)^{2} .
$$

The noise product $\bar{S}_{V V} \bar{S}_{I I}$ is precisely twice the quantumlimited value. As a result, the general theory of Sec. V.F tells us that if one couples an input signal weakly to this amplifier (i.e., $Z_{s} \ll Z_{\text {in }}$ ), it is impossible to reach the quantum limit on the added noise. Thus, while our amplifier is ideal in the scattering mode of operation [cf. Eq. (6.16)], it fails to reach the quantum limit when used in the weak-coupling, op-amp mode of operation. Our amplifier's failure to have ideal quantum noise also means that if we tried to use it to do QND qubit detection, the resulting back-action dephasing would be twice as large as the minimum required by quantum mechanics (cf. Sec. IV.B).

One might object to the above conclusions based on the classical expression for the minimal noise temperature, Eq. (5.76). Unlike the quantum noise constraint of Eq. (5.88), this equation also involves the real part of $\bar{S}_{V I}$, and is optimized by our minimal amplifier. However, this does not mean that one can achieve a noise temperature of $\hbar \omega / 2$ at weak coupling. Recall from Sec. V.F that in the usual process of optimizing the noise temperature one starts by assuming the weak-coupling condition that the source impedance $Z_{s}$ is much smaller than the amplifier input impedance $Z_{\text {in }}$. One then finds that to minimize the noise temperature, $\left|Z_{s}\right|$ should be tuned to match the noise impedance of the amplifier $Z_{N} \equiv \sqrt{\bar{S}_{V V} / \bar{S}_{I I}}$. However, in our minimal bosonic amplifier, it follows from Eqs. (6.18) that $Z_{N}=Z_{\text {in }} / \sqrt{2} \sim Z_{\text {in }}$ : the noise impedance is on the order of the input impedance. Thus, it is impossible to match the source impedance to the noise impedance while at the same time satisfying the weak-coupling condition $Z_{s} \ll Z_{\text {in }}$.

Despite its failings, our amplifier can indeed yield a quantum-limited noise temperature in the op-amp mode of operation if we no longer insist on a weak coupling to the input signal. To see this explicitly, imagine we connected our amplifier to a signal source with source impedance $Z_{s}$. The total output noise of the amplifier, referred back to the signal source, will now have the form

$$
\hat{\tilde{V}}_{\text {tot }}=-\left[Z_{s} Z_{a} /\left(Z_{s}+Z_{a}\right)\right] \hat{\tilde{I}}+\hat{\tilde{V}} .
$$

Note that this classical-looking equation can be rigorously justified within the full quantum theory if one starts with a full description of the amplifier and the signal source (e.g., a parallel LC oscillator attached in parallel to the amplifier input). Plugging in the expressions for $\hat{\tilde{I}}$ and $\hat{\tilde{V}}$, we find

$$
\begin{aligned}
\hat{\tilde{V}}_{\text {tot }} & =\sqrt{\frac{\hbar \omega}{2}}\left[\left(\frac{Z_{s} Z_{a}}{Z_{s}+Z_{a}}\right)\left(\frac{2}{\sqrt{Z_{a}}} \hat{u}_{\text {in }}\right)-\sqrt{Z_{a}}\left(\hat{u}_{\text {in }}-\hat{v}_{\text {in }}^{\dagger}\right)\right] \\
& =\sqrt{\frac{\hbar \omega Z_{a}}{2}}\left[\left(\frac{Z_{s}-Z_{a}}{Z_{s}+Z_{a}}\right) \hat{u}_{\text {in }}-\hat{v}_{\text {in }}^{\dagger}\right] .
\end{aligned}
$$

Thus, if one tunes $Z_{s}$ to $Z_{a}=Z_{\text {in }}$, the mode $\hat{u}_{\text {in }}$ does not contribute to the total added noise, and one reaches the 
quantum limit. Physically speaking, by matching the signal source to the input line the back-action noise described by $\hat{\mathcal{F}}_{a}=\hat{u}_{\text {in }}$ does not feed back into the input of the amplifier. Note that to achieve this matching explicitly requires one to be far from weak coupling. Having $Z_{s}=Z_{a}$ means that when we attach the amplifier to the signal source, we dramatically increase the damping of the signal source.

\section{Why is the op-amp quantum limit not achieved?}

Returning to the more interesting case of a weak amplifier-signal coupling, one might still be puzzled as to why our seemingly ideal amplifier misses the quantum limit. While the mathematics behind Eq. (6.20) is fairly transparent, it is also possible to understand this result heuristically. To that end, note again that the amplifier noise cross correlation $\bar{S}_{I V}$ does not vanish in the largegain limit [cf. Eq. (6.18c)]. Correlations between the two amplifier noises represent a kind of information, as by making use of them, we can improve the performance of the amplifier. It is easy to take advantage of out-of-phase correlations between $\tilde{I}$ and $\tilde{V}$ (i.e., $\operatorname{Im} \bar{S}_{V I}$ ) by simply tuning the phase of the source impedance [cf. Eq. (5.75)]. However, one cannot take advantage of in-phase noise correlations (i.e., $\operatorname{Re} \bar{S}_{V I}$ ) as easily. To take advantage of the information here, one needs to modify the amplifier itself. By feeding back some of the output voltage to the input, one could effectively cancel out some of the backaction current noise $\tilde{I}$ and thus reduce the overall magnitude of $\bar{S}_{I I}$. Hence, the unused information in the cross correlator Re $\bar{S}_{V I}$ represents a kind of wasted information: had we made use of these correlations via a feedback loop, we could have reduced the noise temperature and increased the information provided by our amplifier

The presence of a nonzero $\operatorname{Re} \bar{S}_{V I}$ thus corresponds to wasted information, implying that we cannot reach the quantum limit. Recall that within the linear-response approach we were able to prove rigorously that a largegain amplifier with ideal quantum noise must have $\operatorname{Re} \bar{S}_{V I}=0$ [cf. the discussion following Eq. (5.57)]; thus, a nonvanishing $\operatorname{Re} \bar{S}_{V I}$ rigorously implies that one cannot be at the quantum limit. In Appendix $\mathrm{H}$, we give an explicit demonstration of how feedback may be used to utilize these cross correlations to reach the quantum limit.

Finally, yet another way of seeing that our amplifier does not reach the quantum limit (in the weak-coupling regime) is to realize that this system does not have a well-defined effective temperature. Recall from Sec. V.F that a system with ideal quantum noise [i.e., one that satisfies Eq. (5.88) as an equality] necessarily has the same effective temperature at its input and output ports [cf. Eq. (5.55)]. Here that implies the requirement

$$
\left|\lambda_{V}\right|^{2} \bar{S}_{V V} / Z_{\text {out }}=Z_{\text {in }} \bar{S}_{I I} \equiv 2 k_{B} T_{\text {eff }} .
$$

In contrast, our minimal bosonic amplifier has very different input and output effective temperatures

$$
\begin{aligned}
& 2 k_{B} T_{\text {eff,in }}=Z_{\text {in }} \bar{S}_{I I}=\hbar \omega / 2, \\
& 2 k_{B} T_{\text {eff,out }}=\left|\lambda_{V}\right|^{2} \cdot \bar{S}_{V V} / Z_{\text {out }}=2|G| \hbar \omega .
\end{aligned}
$$

This large difference in effective temperatures means that it is impossible for the system to possess ideal quantum noise, and thus it cannot reach the weak-coupling quantum limit.

While it implies that one is not at the quantum limit, the fact that $T_{\text {eff,in }} \ll T_{\text {eff,out }}$ can nonetheless be viewed as an asset. From a practical point of view, a large $T_{\text {eff,in }}$ can be dangerous. Even though the direct effect of the large $T_{\text {eff,in }}$ is offset by an appropriately weak coupling to the amplifier [see Eq. (5.70) and following discussion], this large $T_{\text {eff.in }}$ can also heat up other degrees of freedom if they couple strongly to the back-action noise of the amplifier. This can in turn lead to unwanted heating of the input system. As $T_{\text {eff,in }}$ is usually constant over a broad range of frequencies, this unwanted heating effect can be quite bad. In the minimal amplifier discussed here, this problem is circumvented by having a small $T_{\text {eff,in }}$. The only price that is paid is that the added noise will be $\sqrt{2}$ the quantum limit value. We discuss this issue further in Sec. VII.

\section{REACHING THE QUANTUM LIMIT IN PRACTICE}

\section{A. Importance of QND measurements}

The fact that QND measurements are repeatable is of fundamental practical importance in overcoming detector inefficiencies (Gambetta et al., 2007). A prototypical example is the electron shelving technique (Nagourney et al., 1986; Sauter et al., 1986) used to measure trapped ions. A related technique is used in present implementations of ion-trap-based quantum computation. Here the (extremely long-lived) hyperfine state of an ion is read out via state-dependent optical fluorescence. With properly chosen circular polarization of the exciting laser, only one hyperfine state fluoresces and the transition is cycling; that is, after fluorescence the ion almost always returns to the same state it was in prior to absorbing the exciting photon. Hence the measurement is QND. Typical experimental parameters (Wineland et al., 1998) allow the cycling transition to produce $N \sim 10^{6}$ fluorescence photons. Given the photomultiplier quantum efficiency and typically small solid angle coverage, only a small number $\bar{n}_{d}$ will be detected on average. The probability of getting zero detections (ignoring dark counts for simplicity) and hence misidentifying the hyperfine state is $P(0)=e^{-\bar{n}_{d}}$. Even for a very poor overall detection efficiency of only $10^{-5}$, we still have $\bar{n}_{d}=10$ and nearly perfect fidelity $F=1-P(0) \approx 0.999955$. It is important to note that the total time available for measurement is not limited by the phase coherence time $\left(T_{2}\right)$ of 
the qubit or by the measurement-induced dephasing (Korotkov, 2001a; Makhlin et al., 2001; Schuster et al., 2005; Gambetta et al., 2006), but rather only by the rate at which the qubit makes real transitions between measurement $\left(\hat{\sigma}_{z}\right)$ eigenstates. In a perfect QND measurement there is no measurement-induced state mixing (Makhlin et al., 2001) and the relaxation rate $1 / T_{1}$ is unaffected by the measurement process.

\section{B. Power matching versus noise matching}

In Sec. V, we saw that an important part of reaching the quantum limit on the added noise of an amplifier (when used in the op-amp mode of operation) is to optimize the coupling strength to the amplifier. For a position detector, this condition corresponds to tuning the strength of the back-action damping $\gamma$ to be much smaller than the intrinsic oscillator damping [cf. Eq. (5.69)]. For a voltage amplifier, this condition corresponds to tuning the impedance of the signal source to be equal to the noise impedance [cf. Eq. (5.77)], an impedance that is much smaller than the amplifier's input impedance [cf. Eq. (5.90)].

In this section, we make the simple point that optimizing the coupling (i.e., source impedance) to reach the quantum limit is not the same as what one would do to optimize the power gain. To understand this, we need to introduce another measure of power gain commonly used in the engineering community, the available power gain $G_{P \text {,avail }}$. For simplicity, we discuss this quantity in the context of a linear voltage amplifier, using the notations of Sec. V.F; it can be analogously defined for the position detector of Sec. V.E. $G_{P \text {,avail }}$ tells us how much power we are providing to an optimally matched output load relative to the maximum power we could in principle have extracted from the source. This is in marked contrast to the power gain $G_{P}$, which was calculated using the actual power drawn at the amplifier input.

For the available power gain, we first consider $P_{\text {in,avail }}$. This is the maximum possible power that could be delivered to the input of the amplifier, assuming we optimized both the value of the input impedance $Z_{\text {in }}$ and the load impedance $Z_{\text {load }}$ while keeping $Z_{s}$ fixed. For simplicity, we take all impedances to be real in our discussion. In general, the power drawn at the input of the amplifier is $P_{\text {in }}=v_{\text {in }}^{2} Z_{\text {in }} /\left(Z_{s}+Z_{\text {in }}\right)^{2}$. Maximizing this over $Z_{\text {in }}$, we obtain the available input power $P_{\text {in,avail }}$,

$$
P_{\text {in,avail }}=v_{\text {in }}^{2} / 4 Z_{s} \text {. }
$$

The maximum occurs for $Z_{\text {in }}=Z_{s}$.

The output power supplied to the load $P_{\text {out }}$ $=v_{\text {load }}^{2} / Z_{\text {load }}$ is calculated as before, keeping $Z_{\text {in }}$ and $Z_{s}$ distinct. One has

$$
P_{\text {out }}=\frac{v_{\text {out }}^{2}}{Z_{\text {load }}}\left(\frac{Z_{\text {load }}}{Z_{\text {out }}+Z_{\text {load }}}\right)^{2}
$$

$$
=\frac{\lambda^{2} v_{\text {in }}^{2}}{Z_{\text {out }}}\left(\frac{Z_{\text {in }}}{Z_{\text {in }}+Z_{s}}\right)^{2} \frac{Z_{\text {load }} / Z_{\text {out }}}{\left(1+Z_{\text {load }} / Z_{\text {out }}\right)^{2}} .
$$

As a function of $Z_{\text {load }}, P_{\text {out }}$ is maximized when $Z_{\text {load }}$ $=Z_{\text {out }}$,

$$
P_{\text {out } \text { max }}=\frac{\lambda^{2} v_{\text {in }}^{2}}{4 Z_{\text {out }}}\left(\frac{Z_{\text {in }}}{Z_{\text {in }}+Z_{s}}\right)^{2} .
$$

The available power gain is now defined as

$$
\begin{aligned}
G_{P, \text { avail }} & \equiv \frac{P_{\text {out }, \text { max }}}{P_{\text {in,avail }}}=\frac{\lambda^{2} Z_{s}}{Z_{\text {out }}}\left(\frac{Z_{\text {in }}}{Z_{\text {in }}+Z_{s}}\right)^{2} \\
& =G_{P} \frac{4 Z_{s} / Z_{\text {in }}}{\left(1+Z_{s} / Z_{\text {in }}\right)^{2}} .
\end{aligned}
$$

We see that $G_{P \text {,avail }}$ is strictly less than or equal to the power gain $G_{P}$; equality is achieved only when $Z_{s}=Z_{\text {in }}$ (i.e., when the source impedance is "power matched" to the input of the amplifier). The general situation where $G_{P, \text { avail }}<G_{P}$ indicates that we are not drawing as much power from the source as we could, and hence the actual power supplied to the load is not as large as it could be.

Consider now a situation where we have achieved the quantum limit on the added noise. This necessarily means that we have "noise matched," i.e., taken $Z_{s}$ to be equal to the noise impedance $Z_{N}$. The available power gain in this case is

$$
G_{P, \text { avail }} \simeq \lambda^{2} Z_{N} / Z_{\text {out }} \simeq 2 \sqrt{G_{P}} \ll G_{P} .
$$

We have used Eq. (5.90), which tells us that the noise impedance is smaller than the input impedance by a large factor of $1 /\left(2 \sqrt{G_{P}}\right)$. Thus, as reaching the quantum limit requires the use of a source impedance much smaller than $Z_{\text {in }}$, it results in a dramatic drop in the available power gain compared to the case where we power match (i.e., take $Z_{s}=Z_{\text {in }}$ ). In practice, one must decide whether it is more important to minimize the added noise or maximize the power provided at the output of the amplifier: one cannot do both at the same time.

\section{CONCLUSIONS}

In this review, we have given an introduction to quantum limits for position detection and amplification, limits that are tied to fundamental constraints on quantum noise correlators. We end by emphasizing notable current developments and pointing out future perspectives in the field.

As emphasized, much of our discussion has been directly relevant to the measurement of mechanical nanoresonators, a topic attracting considerable recent attention. These nanoresonators are typically studied by coupling them either to electrical (often superconducting) circuits or to optical cavities. A key goal is to achieve quantum-limited continuous position detection (cf. Sec. V.E); current experiments are coming tantalizingly close to this limit (cf. Table III). Although the ability to follow the nanoresonator's motion with a precision 
set by the quantum limit is in principle independent from being at low temperatures, it becomes interesting only when the systems are near their ground state; one could then, e.g., monitor the oscillator's zero-point fluctuations (cf. Sec. III.B.3). Given the comparatively small values of mechanical frequencies (mostly less than 1 $\mathrm{GHz}$ ), this often calls for the application of nonequilibrium cooling techniques which exploit back-action to reduce the effective temperature of the mechanical device, a technique that has been demonstrated recently in both superconducting circuits and optomechanical setups [see Marquardt and Girvin (2009) for a review].

The ability to perform quantum-limited position detection will in turn open up many new interesting avenues of research. Among the most significant is the possibility of quantum feedback control (Wiseman and Milburn, 1993, 1994), where one uses the continuously obtained measurement output to tailor the state of the mechanical resonator. The relevant theoretical framework is that of quantum conditional evolution and quantum trajectories (see, e.g., Brun, 2002; Jacobs and Steck, 2006), where one tracks the state of a measured quantum system in a particular run of the experiment. Application of these ideas has only recently been explored in condensed matter contexts (Korotkov, 1999, 2001b; Goan and Milburn, 2001; Goan et al., 2001; Oxtoby et al., 2006, 2008; Bernád et al., 2008). Fully understanding the potential of these techniques, as well as differences that occur in condensed matter versus atomic physics contexts, remains an active area of research. Other important directions in nanomechanics include the possibility of detecting quantum jumps in the state of a mechanical resonator via QND measurement of its energy (Santamore, Doherty, and Cross, 2004; Santamore, Goan, et al., 2004; Jayich et al., 2008; Thompson et al., 2008), as well as the possibility of making back-action-evading measurements (cf. Sec. V.H). Back-action evasion using a microwave cavity detector coupled to a nanomechanical resonator was recently reported (Hertzberg et al., 2010).

Another area distinct from nanomechanics where rapid progress is being made is the readout of solid state qubits using microwave signals sent through cavities whose transmission properties are controlled by the qubit. At the moment, one is close to achieving goodfidelity single-shot QND readout, which is a prerequisite for a large number of applications in quantum information processing. The gradually growing information about the qubit state is extracted from the measured noisy microwave signal trace, leading to a corresponding collapse of the qubit state. This process can also be described by conditional quantum evolution and quantum trajectories.

A promising method for superconducting qubit readout currently employed is a so-called latching measurement, where the hysteretic behavior of a strongly driven anharmonic system (e.g., a Josephson junction) is exploited to toggle between two states depending on the qubit state (Siddiqi et al., 2004; Lupaşcu et al., 2006). Although this is then no longer a linear measurement scheme and is therefore distinct from what was dis- cussed in this review, it can be turned into a linear amplifier for a sufficiently weak input signal. An interesting and important open question is whether such a setup can reach the quantum limit on linear amplification.

Both qubit detection and mechanical measurements in electrical circuits would benefit from quantum-limited on-chip amplifiers. Such amplifiers are now being developed using the tools of circuit quantum electrodynamics, employing Josephson junctions or SQUIDs coupled to microwave transmission line cavities (Bergeal et al., 2008; Castellanos-Beltran et al., 2008). Such an amplifier has already been used to perform continuous position detection with a measurement imprecision below the SQL level (Teufel et al., 2009).

\section{ACKNOWLEDGMENTS}

This work was supported in part by NSERC, the Canadian Institute for Advanced Research (CIFAR), NSA under ARO Contract No. W911NF-05-1-0365, the NSF under Grant No. DMR-0653377 and No. DMR-0603369, the David and Lucile Packard Foundation, the W. M. Keck Foundation, and the Alfred P. Sloan Foundation. We acknowledge the support and hospitality of the Aspen Center for Physics where part of this work was carried out. F.M. acknowledges support via DIP, GIF, and through SFB 631, SFB/TR 12, the Nanosystems Initiative Munich, and the Emmy-Noether program. S.M.G. acknowledges the support of the Centre for Advanced Study at the Norwegian Academy of Science and Letters where part of this work was carried out.

\section{REFERENCES}

Aassime, A., G. Johansson, G. Wendin, R. J. Schoelkopf, and P. Delsing, 2001, Phys. Rev. Lett. 86, 3376.

Abramovici, A., et al., 1992, Science 256, 325.

Aguado, R., and L. P. Kouwenhoven, 2000, Phys. Rev. Lett. 84, 1986.

Aleiner, I. L., N. S. Wingreen, and Y. Meir, 1997, Phys. Rev. Lett. 79, 3740.

Allahverdyan, A. E., R. Balian, and T. M. Nieuwenhuizen, 2001, Phys. Rev. A 64, 032108.

Arcizet, O., P.-F. Cohadon, T. Briant, M. Pinard, and A. Hedimann, 2006, Nature (London) 444, 71.

Armen, M. A., J. K. Au, J. K. Stockton, A. C. Doherty, and H. Mabuchi, 2002, Phys. Rev. Lett. 89, 133602.

Averin, D. V., 2000a, e-print arXiv:cond-mat/0010052.

Averin, D. V., 2000b, Fortschr. Phys. 48, 1055.

Averin, D., 2003, in Quantum Noise in Mesoscopic Systems, edited by Y. Nazarov (Kluwer, Amsterdam), pp. 205-228.

Bergeal, N., R. Vijay, V. E. Manucharyan, I. Siddiqi, R. J. Schoelkopf, S. M. Girvin, and M. H. Devoret, 2008, e-print arXiv:0805.3452, Nature (to be published).

Bernád, J. Z., A. Bodor, T. Geszti, and L. Diósi, 2008, Phys. Rev. B 77, 073311.

Blais, A., R.-S. Huang, A. Wallraff, S. M. Girvin, and R. J. Schoelkopf, 2004, Phys. Rev. A 69, 062320.

Blencowe, M. P., and E. Buks, 2007, Phys. Rev. B 76, 014511. Bocko, M. F., and R. Onofrio, 1996, Rev. Mod. Phys. 68, 755. Bohm, D., 1989, Quantum Theory (Dover, New York). 
Boylestad, R., and L. Nashelsky, 2006, Electronic Devices and Circuit Theory, 9th ed. (Prentice Hall, Engelwood Cliffs, NJ). Braginsky, V. B., and F. Y. Khalili, 1992, Quantum Measurement (Cambridge University Press, Cambridge).

Braginsky, V. B., and F. Y. Khalili, 1996, Rev. Mod. Phys. 68, 1. Braginsky, V. B., Y. I. Vorontsov, and K. P. Thorne, 1980, Science 209, 547.

Brun, T. A., 2002, Am. J. Phys. 70, 719.

Buks, E., R. Schuster, M. Heiblum, D. Mahalu, and V. Umansky, 1998, Nature (London) 391, 871.

Callen, H. B., and T. A. Welton, 1951, Phys. Rev. 83, 34.

Castellanos-Beltran, M. A., K. D. Irwin, G. C. Hilton, L. R. Vale, and K. W. Lehnert, 2008, Nat. Phys. 4, 929.

Caves, C. M., 1982, Phys. Rev. D 26, 1817.

Caves, C. M., and B. L. Schumaker, 1985, Phys. Rev. A 31, 3068.

Caves, C. M., K. S. Thorne, R. W. P. Drever, V. D. Sandberg, and M. Zimmermann, 1980, Rev. Mod. Phys. 52, 341.

Cleland, A. N., J. S. Aldridge, D. C. Driscoll, and A. C. Gossard, 2002, Appl. Phys. Lett. 81, 1699.

Clerk, A. A., 2004, Phys. Rev. B 70, 245306.

Clerk, A. A., 2006, Phys. Rev. Lett. 96, 056801.

Clerk, A. A., M. H. Devoret, S. M. Girvin, F. Marquardt, and R. J. Schoelkopf, 2009, see supplementary material at http:// link.aps.org/supplemental/10.1103/RevModPhys.82.1155 for additional pedagogical appendices which complement the main text of this article.

Clerk, A. A., and S. M. Girvin, 2004, Phys. Rev. B 70, 121303. Clerk, A. A., S. M. Girvin, A. K. Nguyen, and A. D. Stone, 2002, Phys. Rev. Lett. 89, 176804.

Clerk, A. A., S. M. Girvin, and A. D. Stone, 2003, Phys. Rev. B 67, 165324.

Clerk, A. A., F. Marquardt, and K. Jacobs, 2008, New J. Phys. 10, 095010.

Clerk, A. A., and A. D. Stone, 2004, Phys. Rev. B 69, 245303. Corbitt, T., Y. Chen, E. Innerhofer, H. Muller-Ebhardt, D. Ottaway, H. Rehbein, D. Sigg, S. Whitcomb, C. Wipf, and N. Mavalvala, 2007, Phys. Rev. Lett. 98, 150802.

Courty, J. M., F. Grassia, and S. Reynaud, 1999, Europhys. Lett. 46, 31.

Danilov, V. V., K. K. Likharev, and A. B. Zorin, 1983, IEEE Trans. Magn. 19, 572.

Devoret, M. H., and R. J. Schoelkopf, 2000, Nature (London) 406, 1039.

Doherty, A. C., S. Habib, K. Jacobs, H. Mabuchi, and S. M. Tan, 2000, Phys. Rev. A 62, 012105.

Doiron, C. B., B. Trauzettel, and C. Bruder, 2007, Phys. Rev. B 76, 195312.

Duty, T., G. Johansson, K. Bladh, D. Gunnarsson, C. Wilson, and P. Delsing, 2005, Phys. Rev. Lett. 95, 206807.

Dykman, M. I., 1978, Sov. Phys. Solid State 20, 1306.

Etaki, S., M. Poot, I. Mahboob, K. Onomitsu, H. Yamaguchi, and H. S. J. V. der Zant, 2008, Nat. Phys. 4, 785.

Falferi, P., M. Bonaldi, M. Cerdonio, R. Mezzena, G. A. Prodi, A. Vinante, and S. Vitale, 2008, Appl. Phys. Lett. 93, 172506. Flowers-Jacobs, N. E., D. R. Schmidt, and K. W. Lehnert, 2007, Phys. Rev. Lett. 98, 096804.

Gambetta, J., A. Blais, D. I. Schuster, A. Wallraff, and R. J. Schoelkopf, 2006, Phys. Rev. A 74, 042318.

Gambetta, J., W. A. Braff, A. Wallraff, S. M. Girvin, and R. J. Schoelkopf, 2007, Phys. Rev. A 76, 012325.

Gardiner, C. W., and P. Zoller, 2000, Quantum Noise (Springer, Berlin).
Gavish, U., Y. Levinson, and Y. Imry, 2000, Phys. Rev. B 62, (R) 10637.

Gavish, U., B. Yurke, and Y. Imry, 2004, Phys. Rev. Lett. 93, 250601.

Gavish, U., B. Yurke, and Y. Imry, 2006, Phys. Rev. Lett. 96, 133602.

Geremia, J., J. K. Stockton, and H. Mabuchi, 2004, Science 304, 270.

Gerry, C. S., 1985, Phys. Rev. A 31, 2721.

Gigan, S., H. Böhm, M. Paternostro, F. Blaser, J. B. Hertzberg, K. C. Schwab, D. Bauerle, M. Aspelmeyer, and A. Zeilinger, 2006, Nature (London) 444, 67.

Goan, H.-S., and G. J. Milburn, 2001, Phys. Rev. B 64, 235307.

Goan, H. S., G. J. Milburn, H. M. Wiseman, and B. H. Sun, 2001, Phys. Rev. B 63, 125326.

Gordon, J. P., W. H. Louisell, and L. R. Walker, 1963, Phys. Rev. 129, 481.

Gottfried, K., 1966, Quantum Mechanics, Volume I: Fundamentals (Benjamin, Reading, MA).

Grassia, F., 1998, Ph.D. thesis (Universite Pierre et Marie Curie).

Groblacher, S., J. B. Hertzberg, M. R. Vanner, G. D. Cole, S. Gigan, K. C. Schwab, and M. Aspelmeyer, 2009, Nat. Phys. 5, 485.

Gurvitz, S. A., 1997, Phys. Rev. B 56, 15215.

Haroche, S., and J.-M. Raimond, 2006, Exploring the Quantum Atoms, Cavities, and Photons (Oxford University Press, Oxford).

Haus, H. A., 2000, Electromagnetic Noise and Quantum Optical Measurements (Springer, New York).

Haus, H. A., and J. A. Mullen, 1962, Phys. Rev. 128, 2407.

Heffner, H., 1962, Proc. IRE 50, 1604.

Hertzberg, J. B., T. Rocheleau, T. Ndukum, M. Savva, A. A. Clerk, and K. C. Schwab, 2010, Nat. Phys. 6, 213.

Il'ichev, E., N. Oukhanski, A. Izmalkov, T. Wagner, M. Grajcar, H.-G. Meyer, A. Y. Smirnov, A. Maassen van den Brink, M. H. S. Amin, and A. M. Zagoskin, 2003, Phys. Rev. Lett. 91, 097906.

Izmalkov, A., M. Grajcar, E. Il'ichev, T. Wagner, H.-G. Meyer, A. Y. Smirnov, M. H. S. Amin, A. M. van den Brink, and A. M. Zagoskin, 2004, Phys. Rev. Lett. 93, 037003.

Jacobs, K., and D. A. Steck, 2006, Contemp. Phys. 47, 279.

Jayich, A. M., J. C. Sankey, B. M. Zwickl, C. Yang, J. D. Thompson, S. M. Girvin, A. A. Clerk, F. Marquardt, and J. G. E. Harris, 2008, New J. Phys. 10, 095008.

Johansson, G., P. Delsing, K. Bladh, D. Gunnarsson, T. Duty, A. Kack, G. Wendin, and A. Aassime, 2003, in Quantum Noise in Mesoscopic Physics, edited by Y. V. Nazarov (Kluwer, Dordrecht), pp. 337-356.

Johansson, G., A. Käck, and G. Wendin, 2002, Phys. Rev. Lett. 88, 046802.

Johansson, G., L. Tornberg, and C. M. Wilson, 2006, Phys. Rev. B 74, 100504(R).

Jordan, A. N., and M. Büttiker, 2005a, Phys. Rev. Lett. 95, 220401.

Jordan, A. N., and M. Büttiker, 2005b, Phys. Rev. B 71, 125333.

Jordan, A. N., and A. N. Korotkov, 2006, Phys. Rev. B 74, 085307.

Kleckner, D., and D. Bouwmeester, 2006, Nature (London) 444, 75.

Knight, P. L., and V. Buzek, 2004, in Quantum Squeezing, edited by P. D. Drummond, and Z. Ficek (Springer, Berlin), pp. 
3-32.

Knobel, R. G., and A. N. Cleland, 2003, Nature (London) 424, 291.

Koch, R. H., D. J. van Harlingen, and J. Clarke, 1981, Appl. Phys. Lett. 38, 380.

Korotkov, A. N., 1999, Phys. Rev. B 60, 5737.

Korotkov, A. N., 2001a, Phys. Rev. B 63, 085312.

Korotkov, A. N., 2001b, Phys. Rev. B 63, 115403.

Korotkov, A. N., and D. V. Averin, 2001, Phys. Rev. B 64, 165310.

Korotkov, A. N., and M. A. Paalanen, 1999, Appl. Phys. Lett. 74, 4052 .

LaHaye, M. D., O. Buu, B. Camarota, and K. C. Schwab, 2004, Science 304, 74.

Levinson, Y., 1997, Europhys. Lett. 39, 299.

Louisell, W. H., A. Yariv, and A. E. Siegman, 1961, Phys. Rev. 124, 1646.

Lupaşcu, A., E. F. C. Driessen, L. Roschier, C. J. P. M. Harmans, and J. E. Mooij, 2006, Phys. Rev. Lett. 96, 127003.

Lupaşcu, A., C. J. P. M. Harmans, and J. E. Mooij, 2005, Phys. Rev. B 71, 184506.

Lupaşcu, A., S. Saito, T. Picot, P. C. de Groot, C. J. P. M. Harmans, and J. E. Mooij, 2007, Nat. Phys. 3, 119.

Lupaşcu, A., C. J. M. Verwijs, R. N. Schouten, C. J. P. M. Harmans, and J. E. Mooij, 2004, Phys. Rev. Lett. 93, 177006. Makhlin, Y., G. Schön, and A. Shnirman, 2000, Phys. Rev. Lett. 85, 4578

Makhlin, Y., G. Schon, and A. Shnirman, 2001, Rev. Mod. Phys. 73, 357.

Marquardt, F., and S. M. Girvin, 2009, Phys. 2, 40.

Mollow, B. R., and R. J. Glauber, 1967a, Phys. Rev. 160, 1076.

Mollow, B. R., and R. J. Glauber, 1967b, Phys. Rev. 160, 1097.

Mozyrsky, D., I. Martin, and M. B. Hastings, 2004, Phys. Rev. Lett. 92, 018303.

Mück, M., J. B. Kycia, and J. Clarke, 2001, Appl. Phys. Lett. 78, 967.

Nagourney, W., J. Sandberg, and H. Dehmelt, 1986, Phys. Rev. Lett. 56, 2797.

Naik, A., O. Buu, M. D. LaHaye, A. D. Armour, A. A. Clerk, M. P. Blencowe, and K. C. Schwab, 2006, Nature (London) 443, 193.

Nogues, G., A. Rauschenbeutel, S. Osnaghi, M. Brune, J. M. Raimond, and S. Haroche, 1999, Nature (London) 400, 239.

Oxtoby, N. P., J. Gambetta, and H. M. Wiseman, 2008, Phys. Rev. B 77, 125304.

Oxtoby, N. P., H. M. Wiseman, and H.-B. Sun, 2006, Phys. Rev. B 74, 045328.

Pathria, R. K., 1996, Statistical Mechanics, 2nd ed. (Elsevier, Amsterdam).

Peres, A., 1993, Quantum Theory: Concepts and Methods (Kluwer, Dordrecht).

Pilgram, S., and M. Büttiker, 2002, Phys. Rev. Lett. 89, 200401.

Poggio, M., M. P. Jura, C. L. Degen, M. A. Topinka, H. J. Mamin, D. Goldhaber-Gordon, and D. Rugar, 2008, Nat. Phys. 4, 635.

Regal, C. A., J. D. Teufel, and K. W. Lehnert, 2008, Nat. Phys. 4, 555.

Rugar, D., R. Budakian, H. J. Mamin, and B. W. Chui, 2004, Nature (London) 430, 329.

Ruskov, R., and A. N. Korotkov, 2002, Phys. Rev. B 66 , 041401.

Ruskov, R., K. Schwab, and A. N. Korotkov, 2005, Phys. Rev. B 71, 235407.
Santamore, D. H., A. C. Doherty, and M. C. Cross, 2004, Phys. Rev. B 70, 144301.

Santamore, D. H., H.-S. Goan, G. J. Milburn, and M. L. Roukes, 2004, Phys. Rev. A 70, 052105.

Sauter, T., W. Neuhauser, R. Blatt, and P. E. Toschek, 1986, Phys. Rev. Lett. 57, 1696.

Schliesser, A., O. Arcizet, R. Riviere, G. Anetsberger, and T. J. Kippenberg, 2009, Nat. Phys. 5, 509.

Schliesser, A., P. Del'Haye, N. Nooshi, K. J. Vahala, and T. J. Kippenberg, 2006, Phys. Rev. Lett. 97, 243905.

Schliesser, A., R. Rivière, G. Anetsberger, O. Arcizet, and T. J. Kippenberg, 2008, Nat. Phys. 4, 415.

Schoelkopf, R. J., A. A. Clerk, S. M. Girvin, K. W. Lehnert, and M. H. Devoret, 2003, in Quantum Noise in Mesoscopic Physics, edited by Y. V. Nazarov (Kluwer, Dordrecht), pp. 175-203.

Schoelkopf, R. J., P. Wahlgren, A. A. Kozhevnikov, P. Delsing, and D. E. Prober, 1998, Science 280, 1238.

Schuster, D. I., A. Wallraff, A. Blais, L. Frunzio, R.-S. Huang, J. Majer, S. M. Girvin, and R. J. Schoelkopf, 2005, Phys. Rev. Lett. 94, 123602.

Schwinger, J., 1961, J. Math. Phys. 2, 407.

Shnirman, A., and G. Schön, 1998, Phys. Rev. B 57, 15400.

Siddiqi, I., R. Vijay, F. Pierre, C. M. Wilson, M. Metcalfe, C. Rigetti, L. Frunzio, and M. H. Devoret, 2004, Phys. Rev. Lett. 93, 207002.

Sillanpää, M. A., T. Lehtinen, A. Paila, Y. Makhlin, L. Roschier, and P. J. Hakonen, 2005, Phys. Rev. Lett. 95, 206806.

Sprinzak, D., E. Buks, M. Heiblum, and H. Shtrikman, 2000, Phys. Rev. Lett. 84, 5820.

Stern, A., Y. Aharonov, and Y. Imry, 1990, Phys. Rev. A 41, 3436.

Teufel, J. D., T. Donner, M. A. Castellanos-Beltran, J. W. Harlow, and K. W. Lehnert, 2009, e-print arXiv:0906.1212.

Teufel, J. D., C. A. Regal, and K. W. Lehnert, 2008, New J. Phys. 10, 095002.

Thalakulam, M., Z. Ji, and A. J. Rimberg, 2004, Phys. Rev. Lett. 93, 066804.

Thompson, J. D., B. M. Zwickl, A. M. Jayich, F. Marquardt, S. M. Girvin, and J. G. E. Harris, 2008, Nature (London) 452, 72.

Thorne, K. S., R. W. P. Drever, C. M. Caves, M. Zimmermann, and V. D. Sandberg, 1978, Phys. Rev. Lett. 40, 667.

Tittonen, I., G. Breitenbach, T. Kalkbrenner, T. Muller, R. Conradt, S. Schiller, E. Steinsland, N. Blanc, and N. F. de Rooij, 1999, Phys. Rev. A 59, 1038.

Turek, B. A., K. W. Lehnert, A. Clerk, D. Gunnarsson, K. Bladh, P. Delsing, and R. J. Schoelkopf, 2005, Phys. Rev. B 71, 193304.

van den Brink, A. M., 2002, Europhys. Lett. 58, 562.

Vinante, A., R. Mezzena, G. A. Prodi, S. Vitale, M. Cerdonio, P. Falferi, and M. Bonaldi, 2001, Appl. Phys. Lett. 79, 2597. von Neumann, J., 1932, Mathematische Grundlagen der Quantenmechanik (Springer, Berlin).

Wallraff, A., D. I. Schuster, A. Blais, L. Frunzio, R.-S. Huang, J. Majer, S. Kumar, S. M. Girvin, and R. J. Schoelkopf, 2004, Nature (London) 431, 162.

Walls, D. F., and G. J. Milburn, 1994, Quantum Optics (Springer, Berlin).

Weiss, U., 1999, Quantum Dissipative Systems, 2nd ed. (World Scientific, Singapore).

Wellstood, F. C., C. Urbina, and J. Clarke, 1994, Phys. Rev. B 
49, 5942.

Wheeler, J. A., and W. H. Zurek, 1984, Quantum Theory and Measurement (Princeton University Press, Princeton).

Wigner, E. P., 1955, Phys. Rev. 98, 145.

Wineland, D. J., C. Monroe, W. M. Itano, D. Leibfried, B. King, and D. M. Meekhof, 1998, J. Res. Natl. Inst. Stand. Technol. 103, 259.
Wiseman, H. M., and G. J. Milburn, 1993, Phys. Rev. Lett. 70, 548.

Wiseman, H. M., and G. J. Milburn, 1994, Phys. Rev. A 49, 1350.

Yurke, B., L. R. Corruccini, P. G. Kaminsky, L. W. Rupp, A. D. Smith, A. H. Silver, R. W. Simon, and E. A. Whittaker, 1989, Phys. Rev. A 39, 2519. 